\title{
Capture and Reactivity of an Elusive Carbon-Sulfur Centered Biradical
}

\author{
Dennis Gerbig, Bastian Bernhardt, Raffael C. Wende, and Peter R. Schreiner \\ Institute of Organic Chemistry, Justus-Liebig University, Heinrich-Buff-Ring 17, 35392 Giessen, Germany.
}

\section{Supporting Information - Part A: Matrix Isolation Spectra \& Analytical Data}

\section{Table of Contents}

1. Matrix isolation experiments on $p$-nitrobenzaldehyde $(p$-NBA) dithiane............................................................ 2

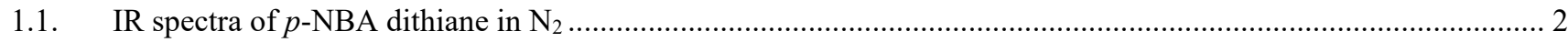

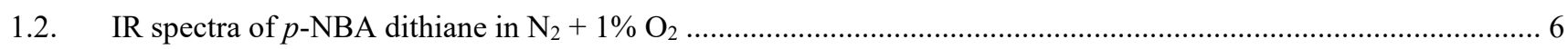

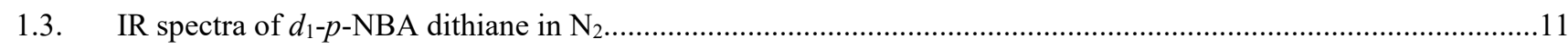

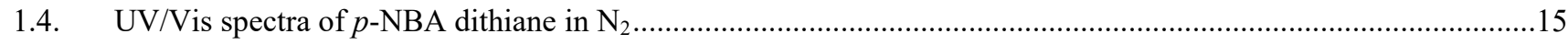

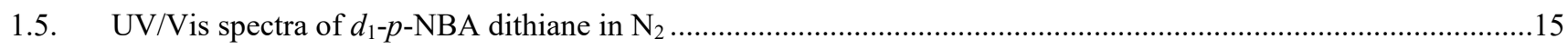

2. Matrix isolation experiments on $p$-nitrobenzaldehyde dimethyl bisthioacetal $(p$-NBA-DMTA) ..............................16

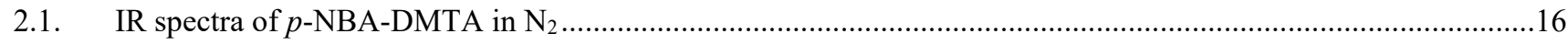

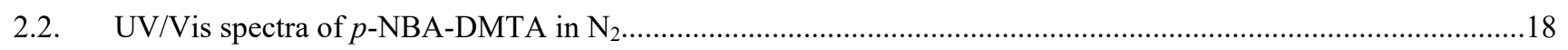

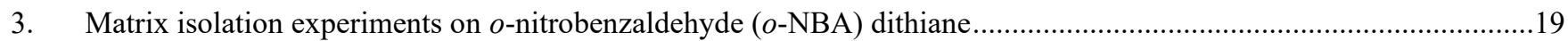

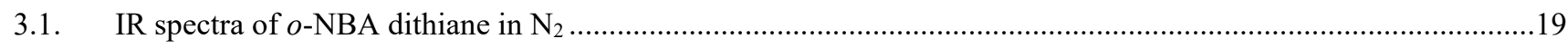

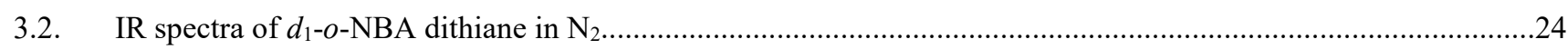

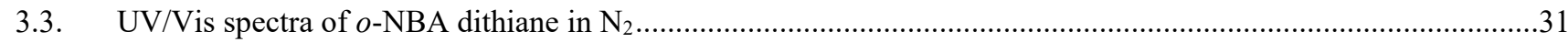

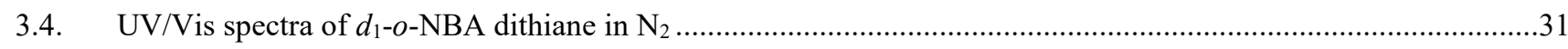

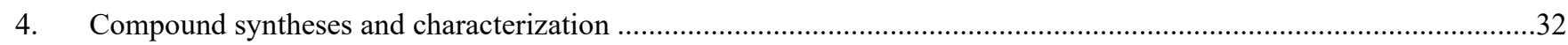

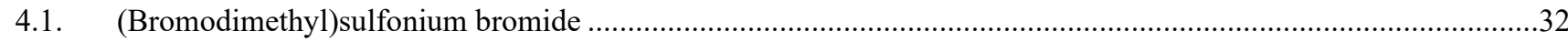

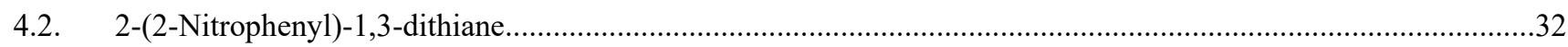

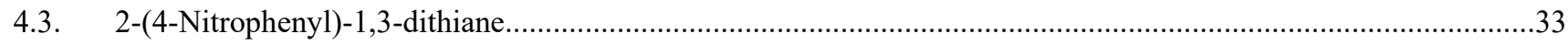

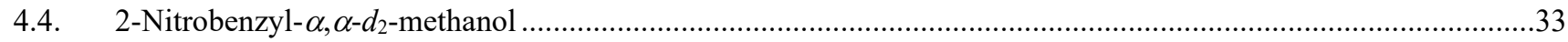

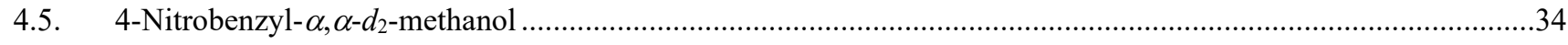

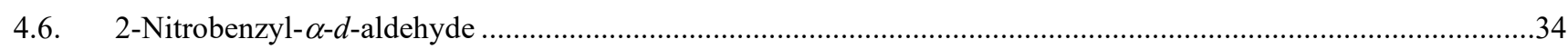

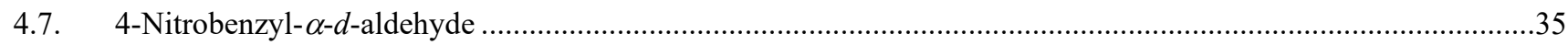

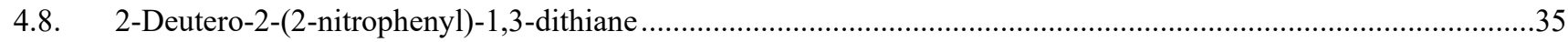

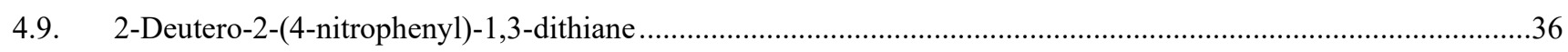

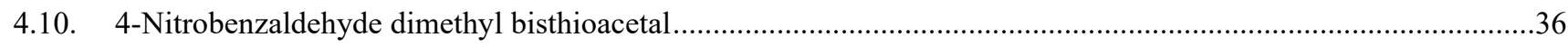


1. Matrix isolation experiments on $p$-nitrobenzaldehyde ( $p$-NBA) dithiane

1.1. IR spectra of $p$-NBA dithiane in $\mathrm{N}_{2}$

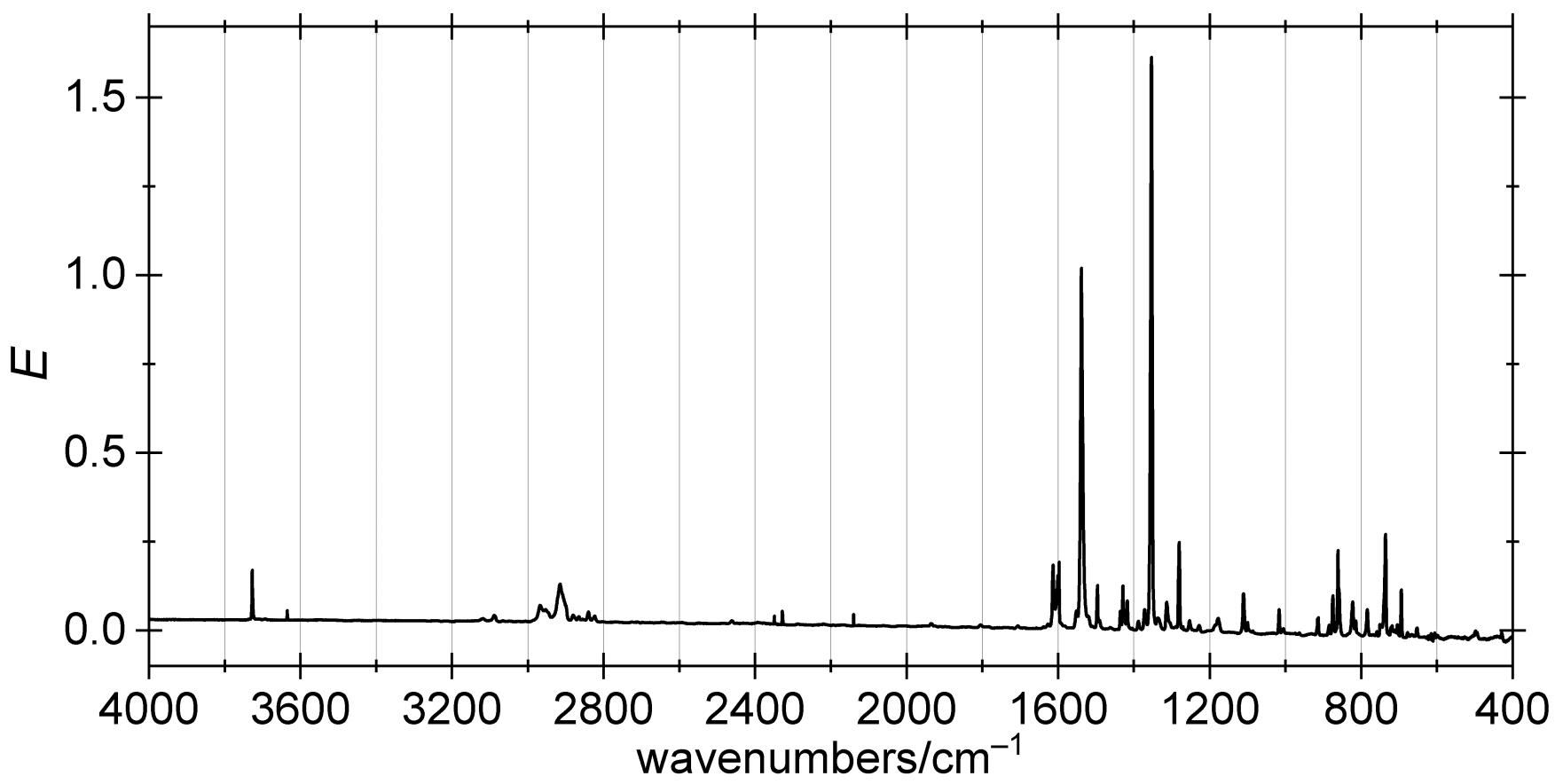

Figure S1: Matrix-isolated $p$-NBA dithiane in $\mathrm{N}_{2}$ at $3 \mathrm{~K}$.

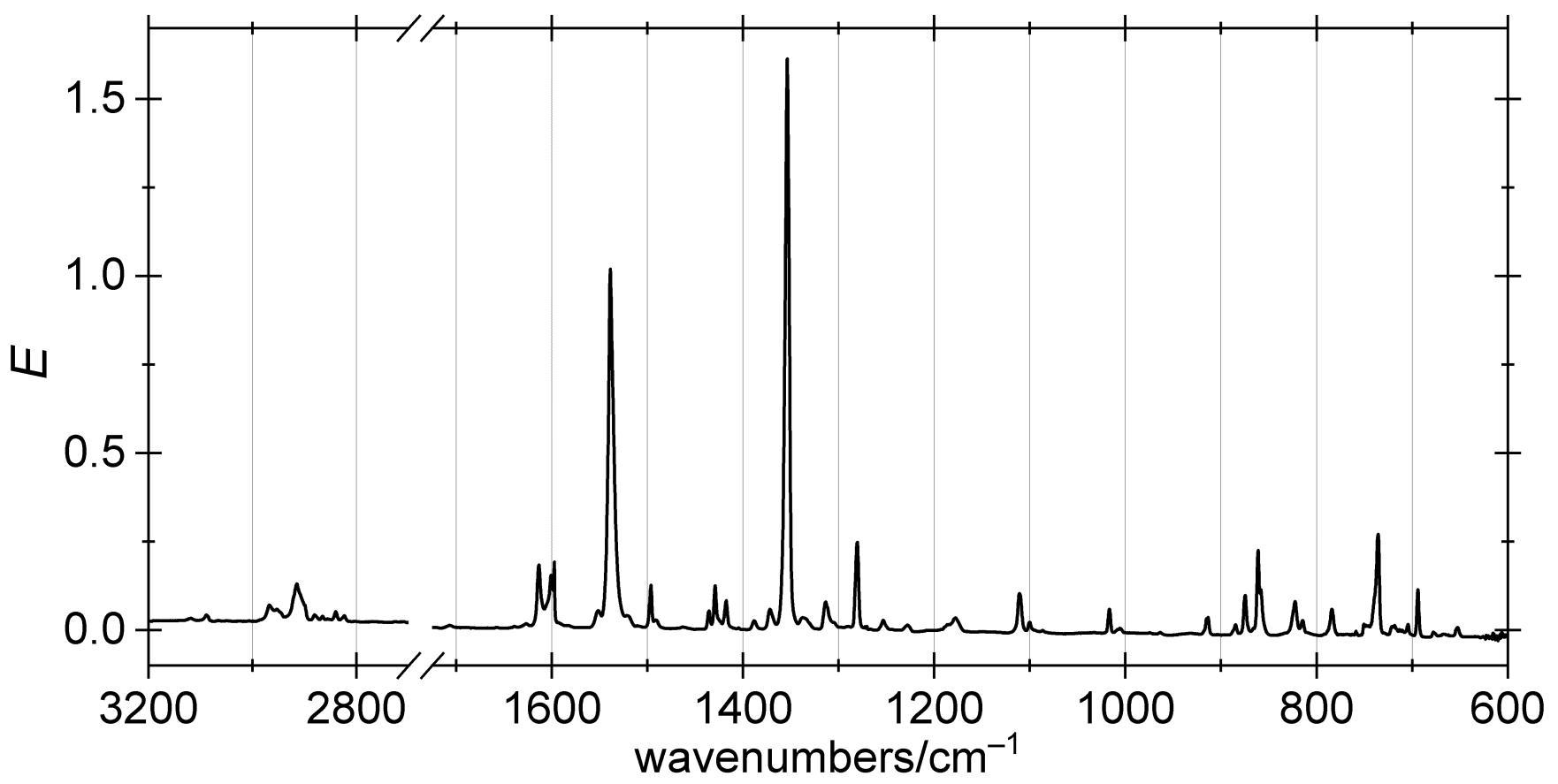

Figure S2: Matrix-isolated $p$-NBA dithiane in $\mathrm{N}_{2}$ at $3 \mathrm{~K}$ (detail). 


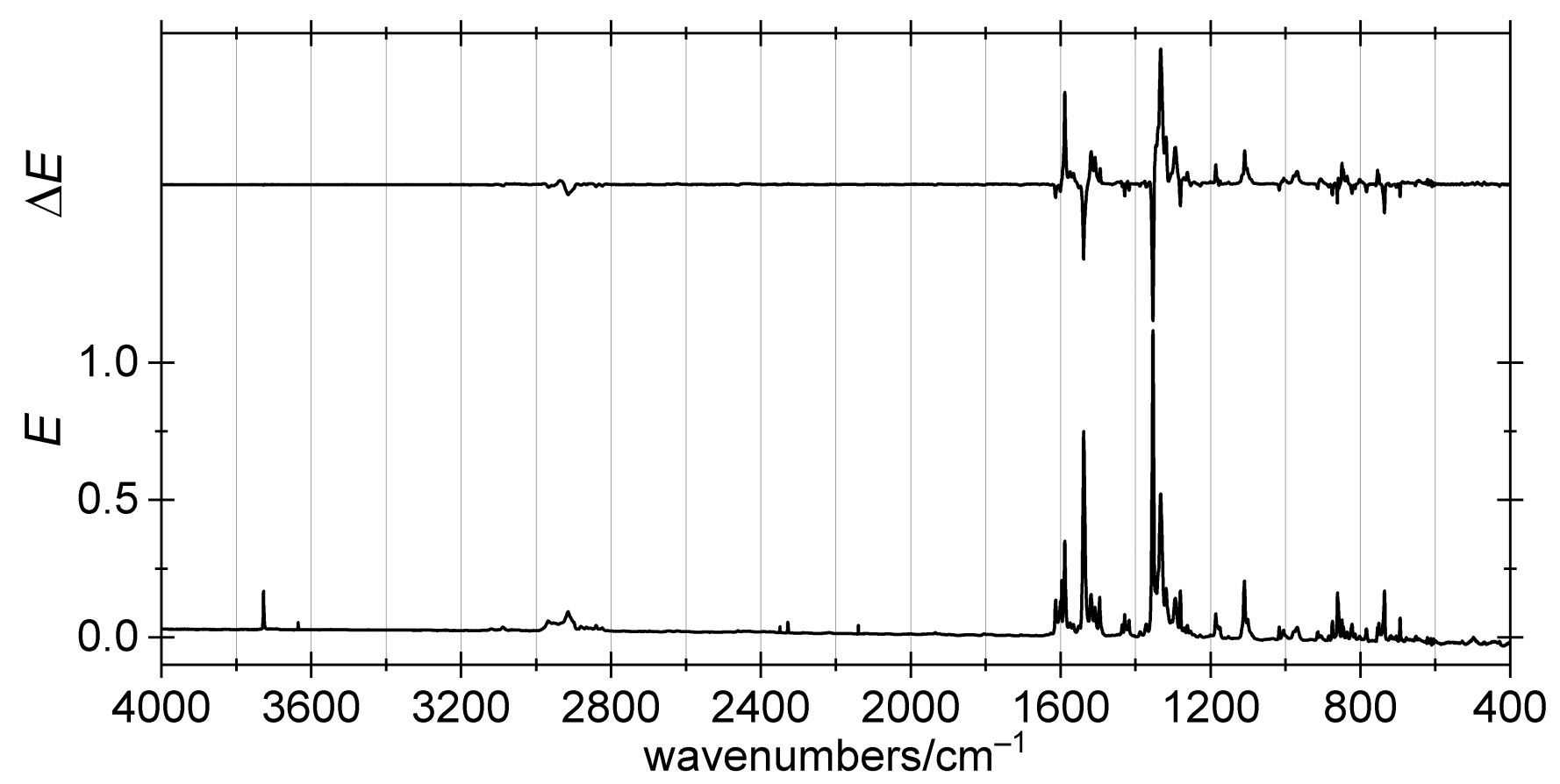

Figure S3: Matrix-isolated $p$-NBA dithiane in $\mathrm{N}_{2}$ at $3 \mathrm{~K}$ after $60 \mathrm{~s}$ irradiation at $313 \mathrm{~nm}$; difference trace (before/after irradiation at $313 \mathrm{~nm}$ ) on top.

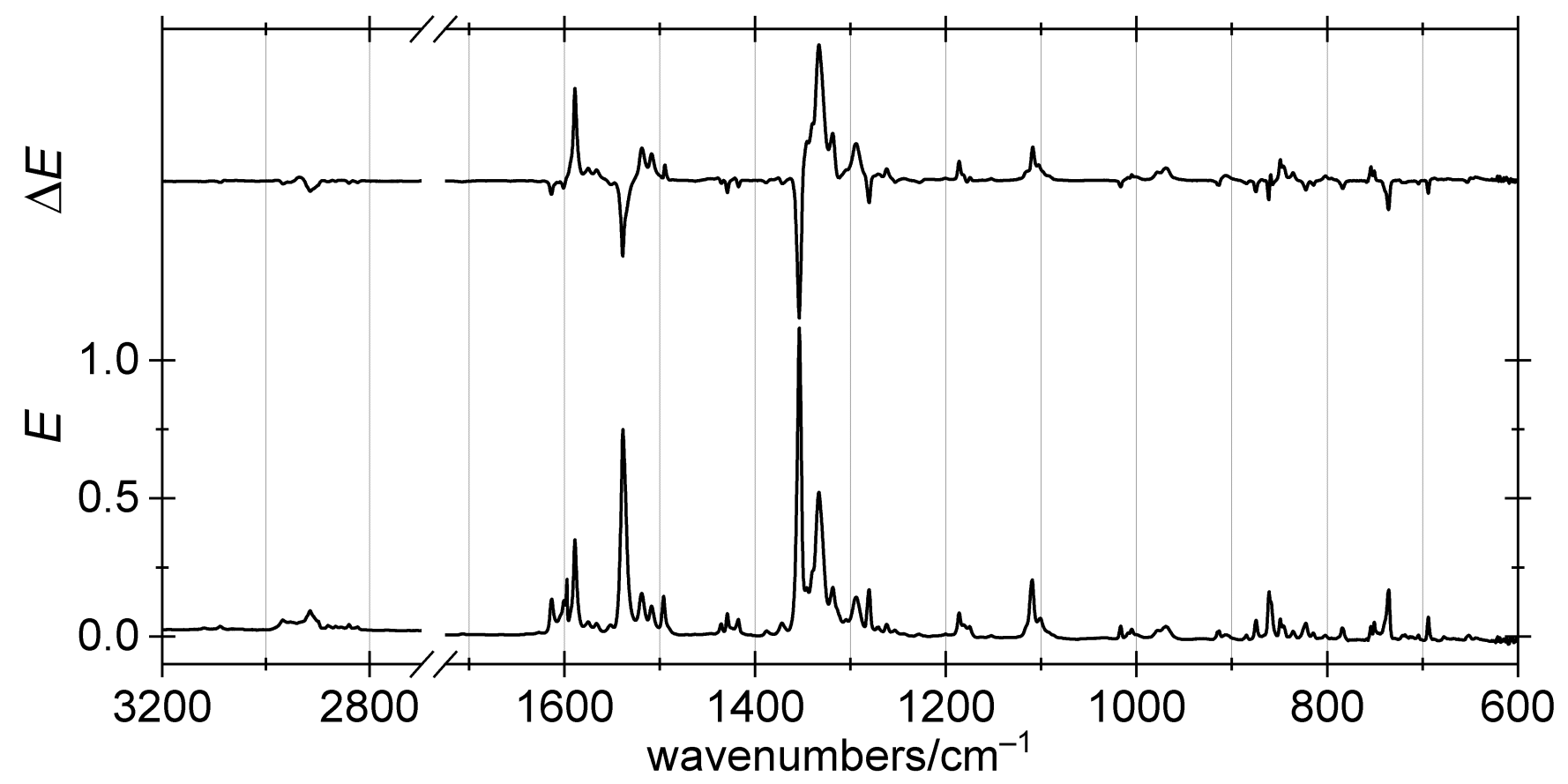

Figure S4: Matrix-isolated $p$-NBA dithiane in $\mathrm{N}_{2}$ at $3 \mathrm{~K}$ after $60 \mathrm{~s}$ irradiation at $313 \mathrm{~nm}$ (detail); difference trace (before/after irradiation at $313 \mathrm{~nm}$ ) on top. 


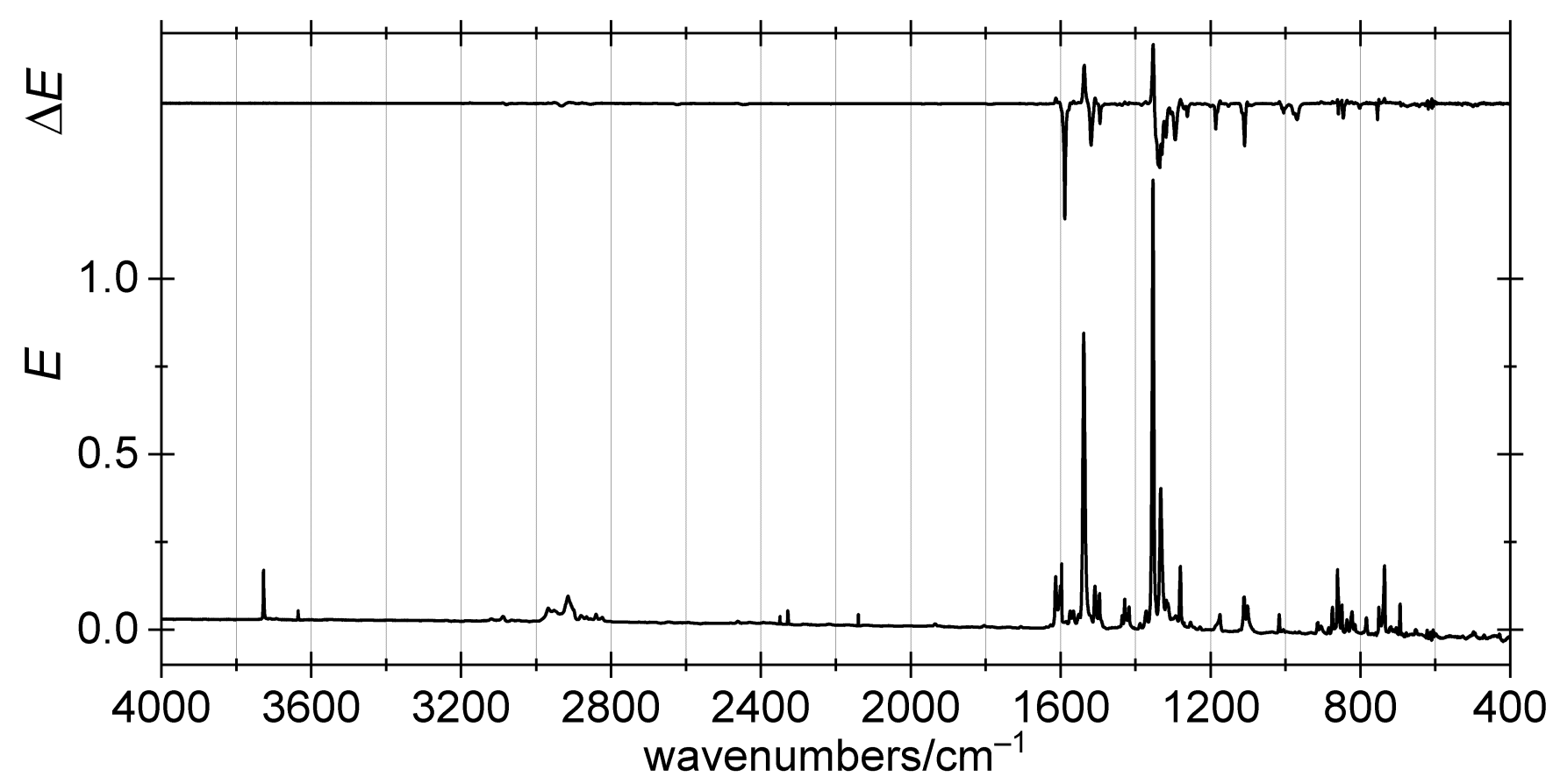

Figure S5: Matrix-isolated $p$-NBA dithiane in $\mathrm{N}_{2}$ at $3 \mathrm{~K}$ after $60 \mathrm{~s}$ irradiation at $313 \mathrm{~nm}$ and $90 \mathrm{~s}$ irradiation at $436 \mathrm{~nm}$; difference trace (before/after irradiation at $436 \mathrm{~nm}$ ) on top.

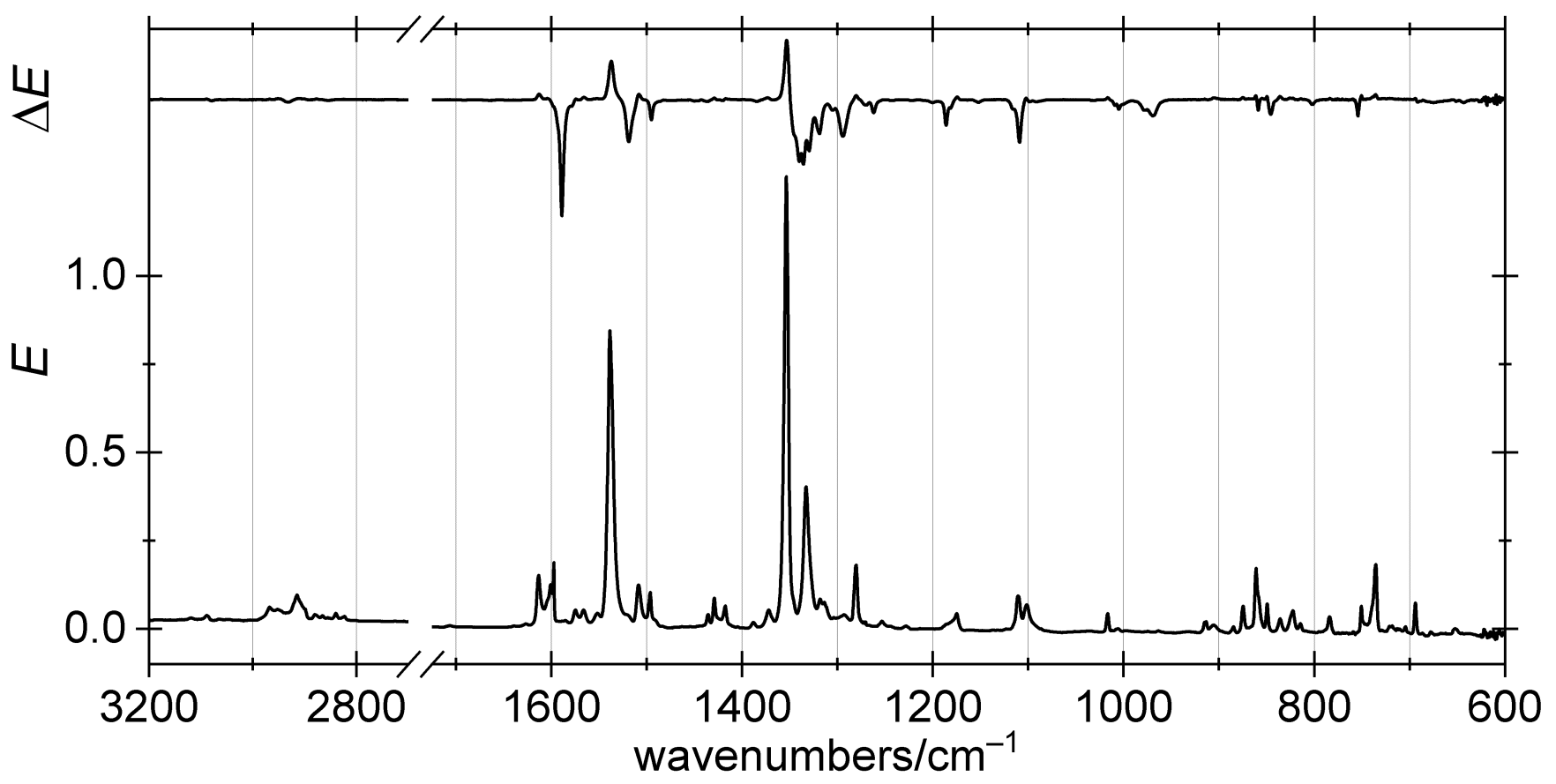

Figure S6: Matrix-isolated $p$-NBA dithiane in $\mathrm{N}_{2}$ at $3 \mathrm{~K}$ after $60 \mathrm{~s}$ irradiation at $313 \mathrm{~nm}$ and $90 \mathrm{~s}$ irradiation at $436 \mathrm{~nm}$ (detail); difference trace (before/after irradiation at $436 \mathrm{~nm}$ ) on top. 


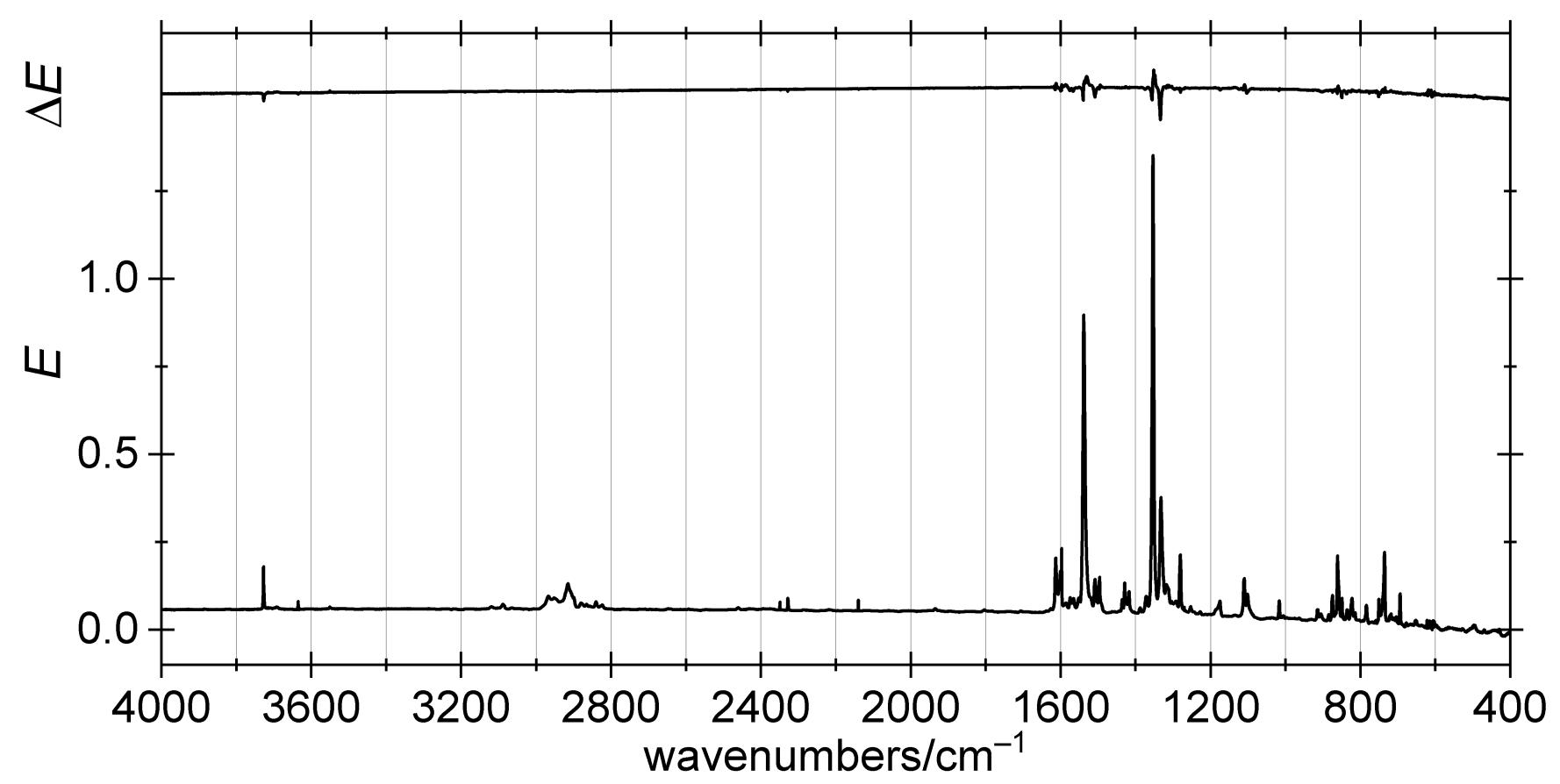

Figure S7: Matrix-isolated $p$-NBA dithiane in $\mathrm{N}_{2}$ at $3 \mathrm{~K}$ after $60 \mathrm{~s}$ irradiation at $313 \mathrm{~nm}, 90 \mathrm{~s}$ irradiation at $436 \mathrm{~nm}$, and subsequent annealing to $27 \mathrm{~K}$; difference trace (before/after annealing to $27 \mathrm{~K}$ ) on top.

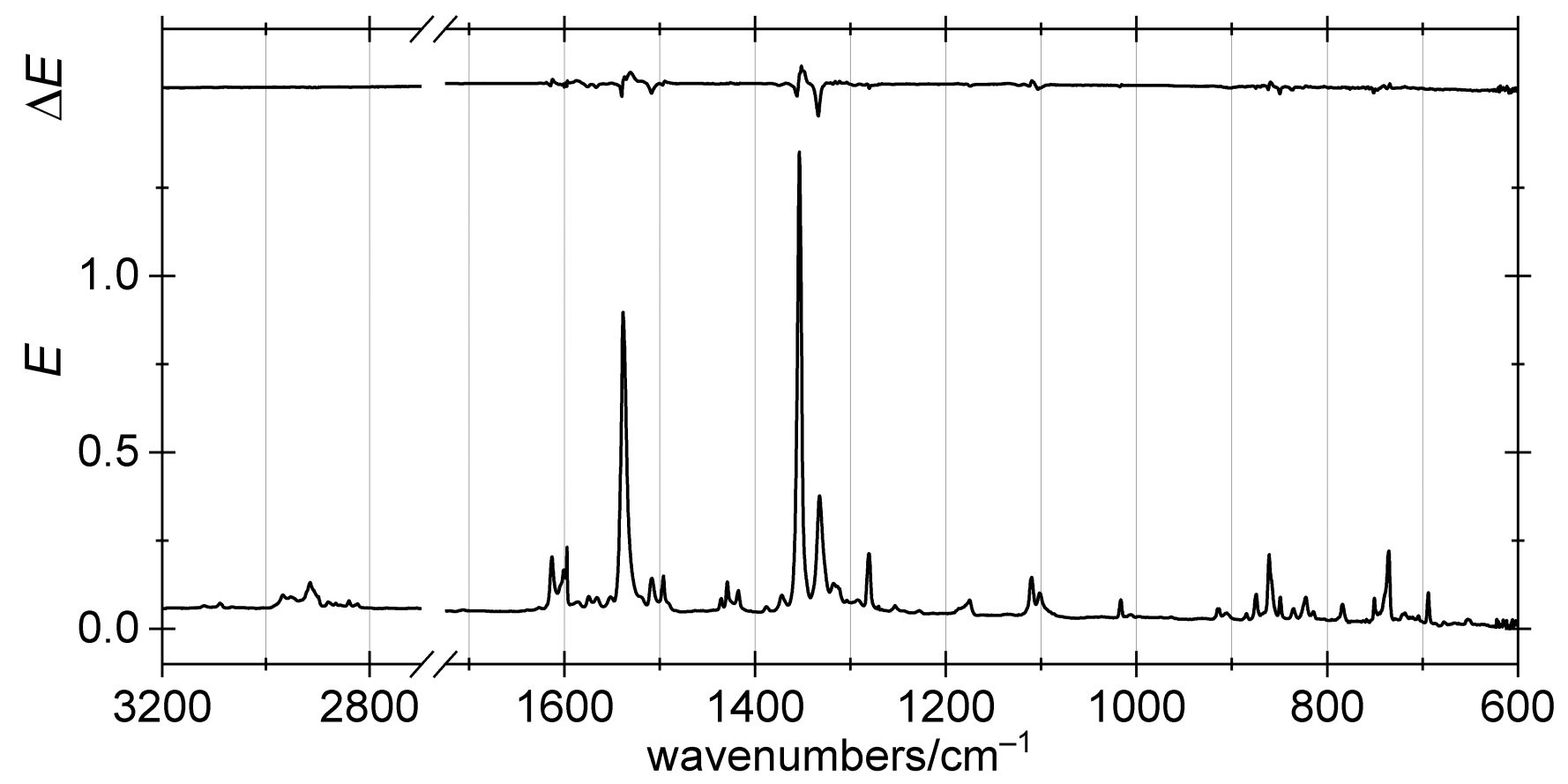

Figure S8: Matrix-isolated $p$-NBA dithiane in $\mathrm{N}_{2}$ at $3 \mathrm{~K}$ after $60 \mathrm{~s}$ irradiation at $313 \mathrm{~nm}, 90 \mathrm{~s}$ irradiation at $436 \mathrm{~nm}$, and subsequent annealing to $27 \mathrm{~K}$ (detail); difference trace (before/after annealing to $27 \mathrm{~K}$ ) on top. 
1.2. IR spectra of $p$-NBA dithiane in $\mathrm{N}_{2}+1 \% \mathrm{O}_{2}$

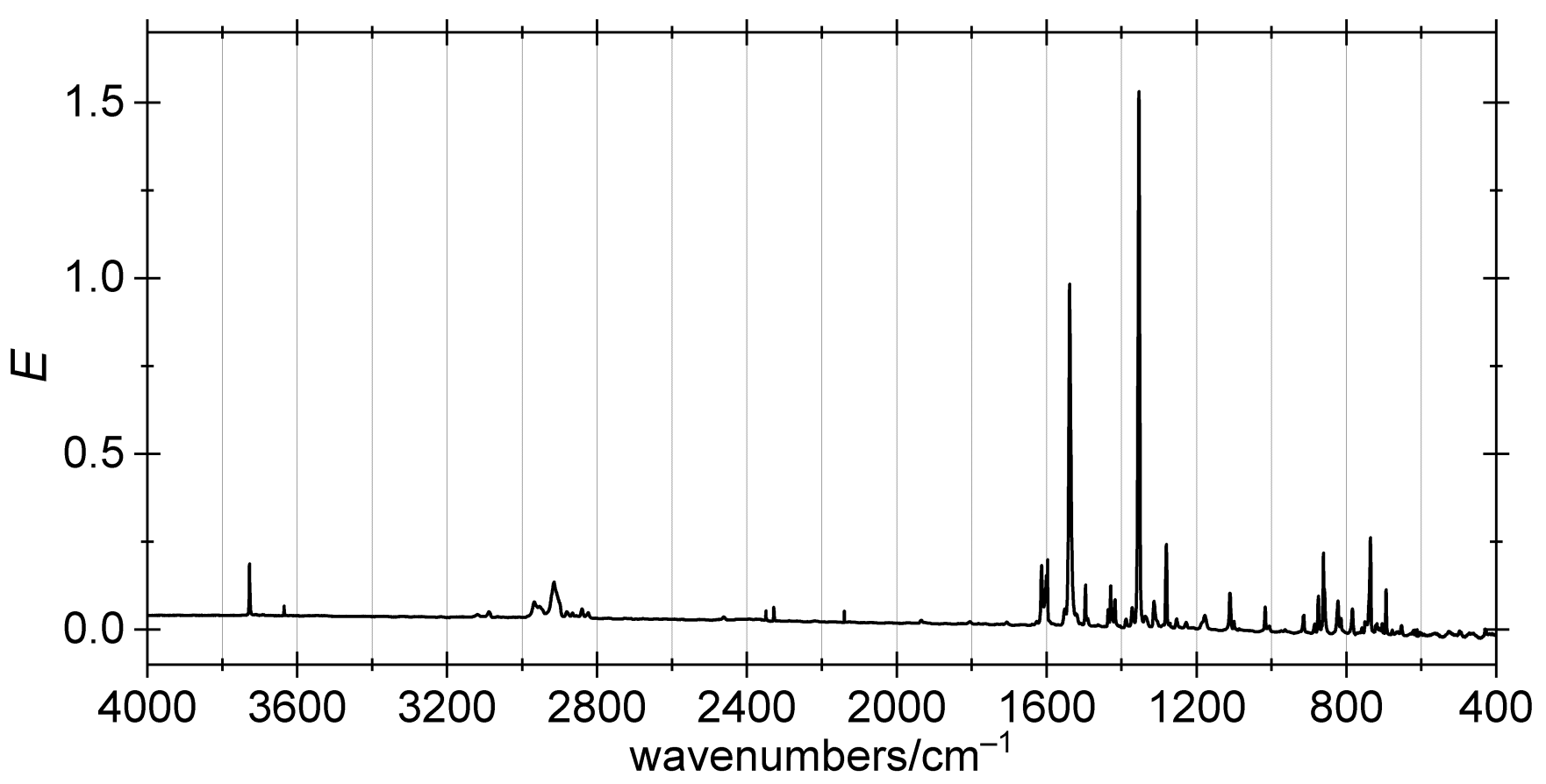

Figure S9: Matrix-isolated $p$-NBA dithiane in $\mathrm{N}_{2}+1 \% \mathrm{O}_{2}$ at $3 \mathrm{~K}$.

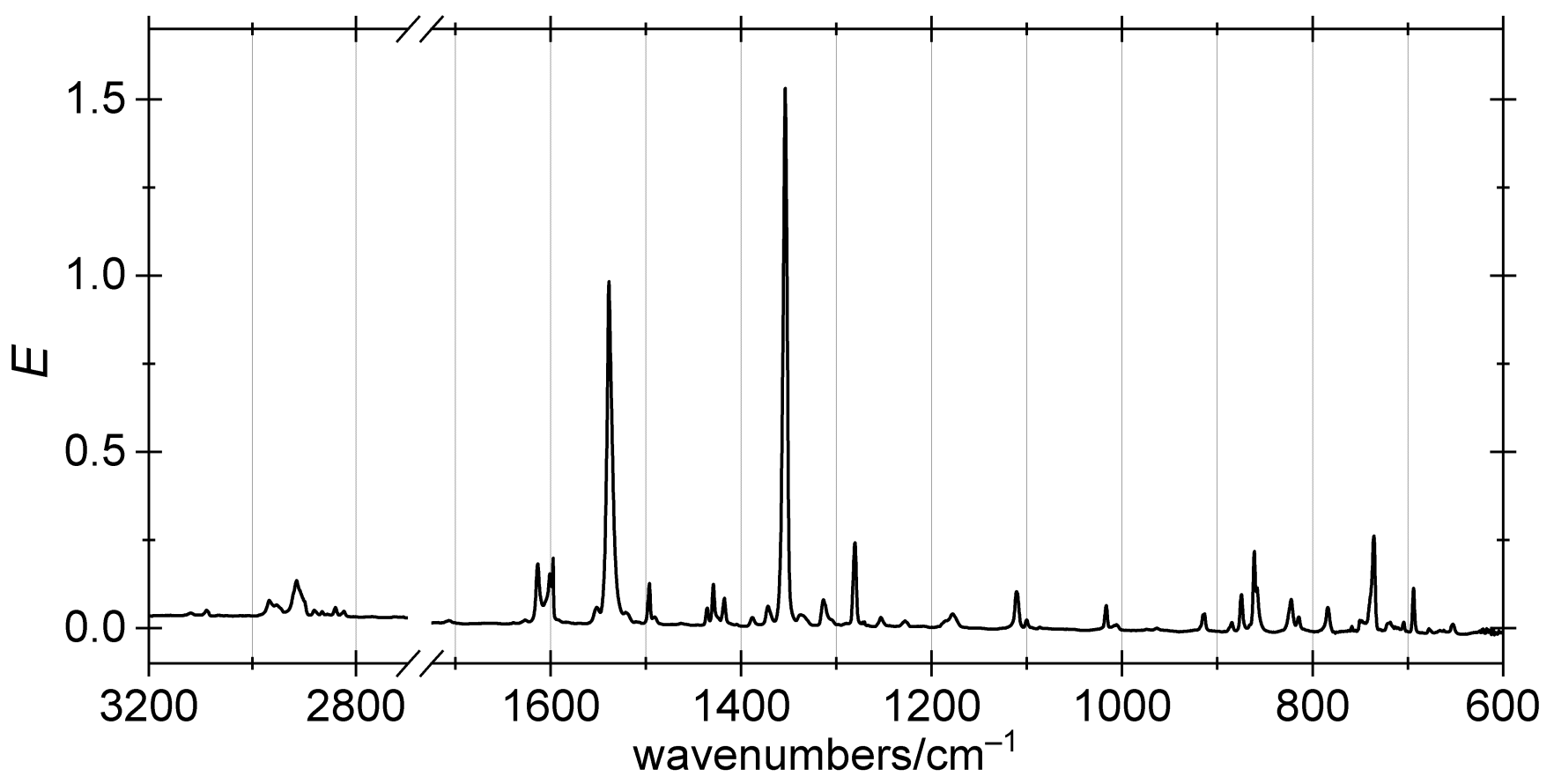

Figure S10: Matrix-isolated $p$-NBA dithiane in $\mathrm{N}_{2}+1 \% \mathrm{O}_{2}$ at $3 \mathrm{~K}$ (detail). 


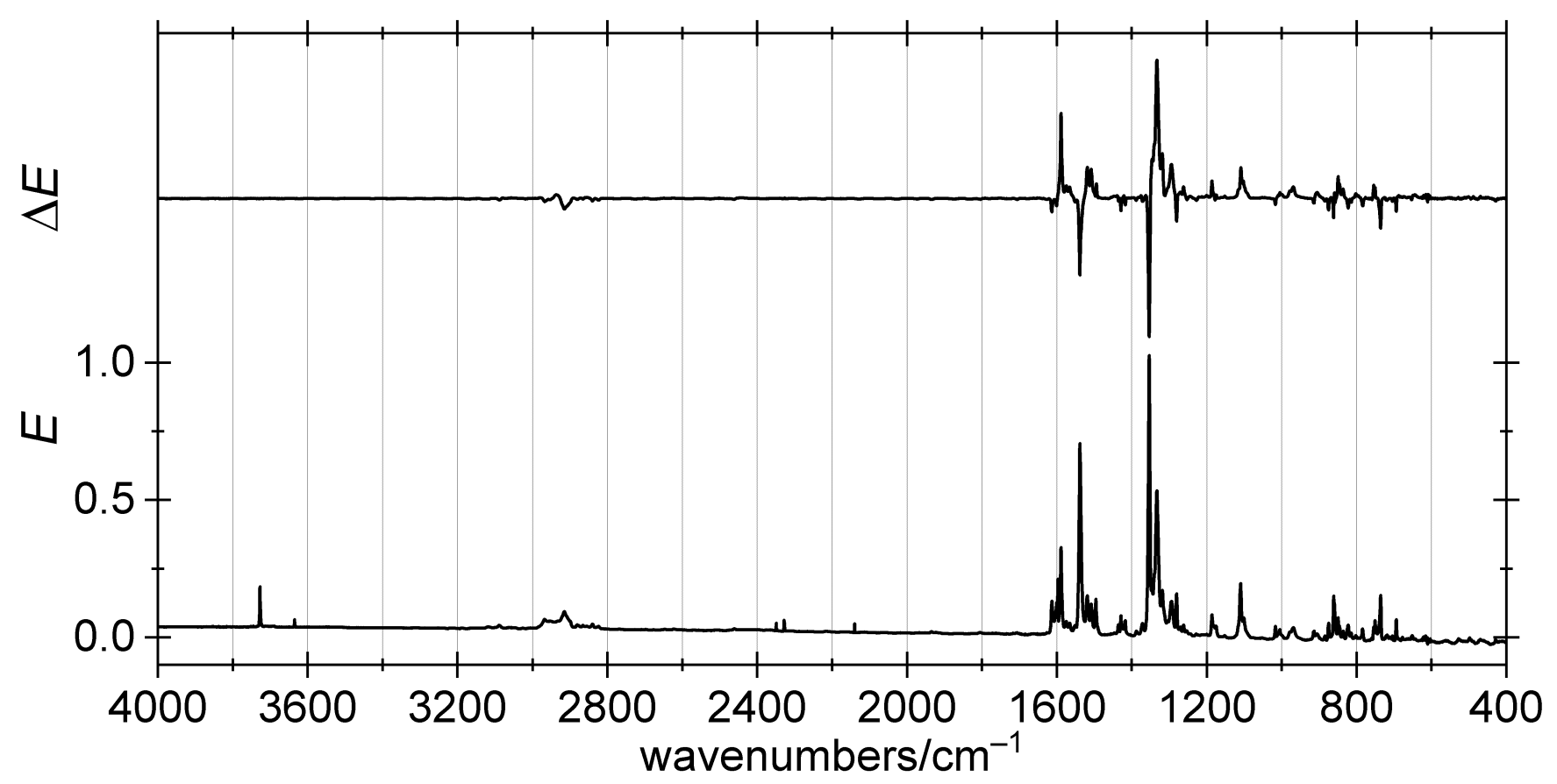

Figure S11: Matrix-isolated $p$-NBA dithiane in $\mathrm{N}_{2}+1 \% \mathrm{O}_{2}$ at $3 \mathrm{~K}$ after $60 \mathrm{~s}$ irradiation at 313 nm; difference trace (before/after irradiation at $313 \mathrm{~nm}$ ) on top.

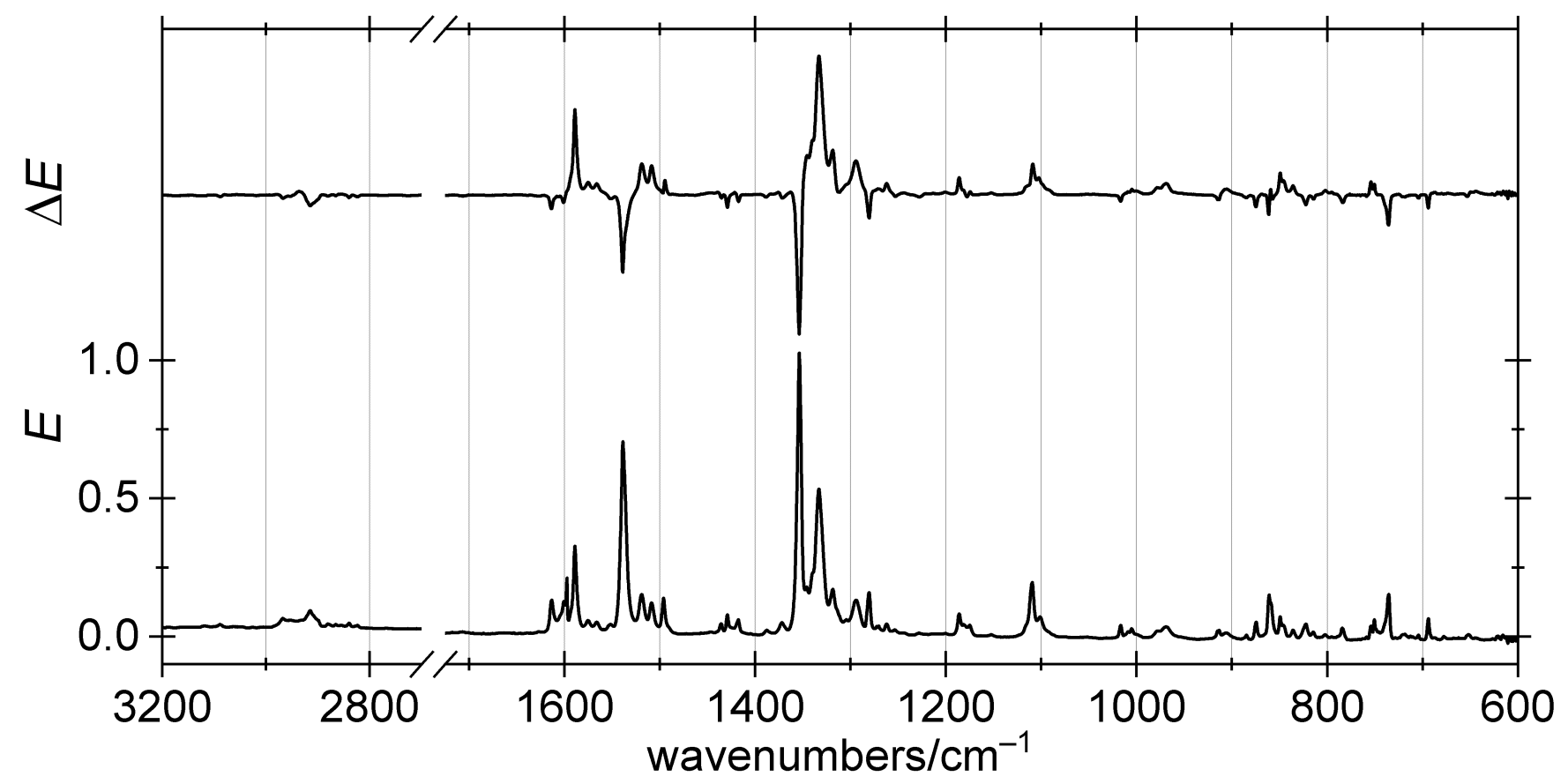

Figure S12: Matrix-isolated $p$-NBA dithiane in $\mathrm{N}_{2}+1 \% \mathrm{O}_{2}$ at $3 \mathrm{~K}$ after $60 \mathrm{~s}$ irradiation at $313 \mathrm{~nm}$ (detail); difference trace (before/after irradiation at $313 \mathrm{~nm}$ ) on top. 


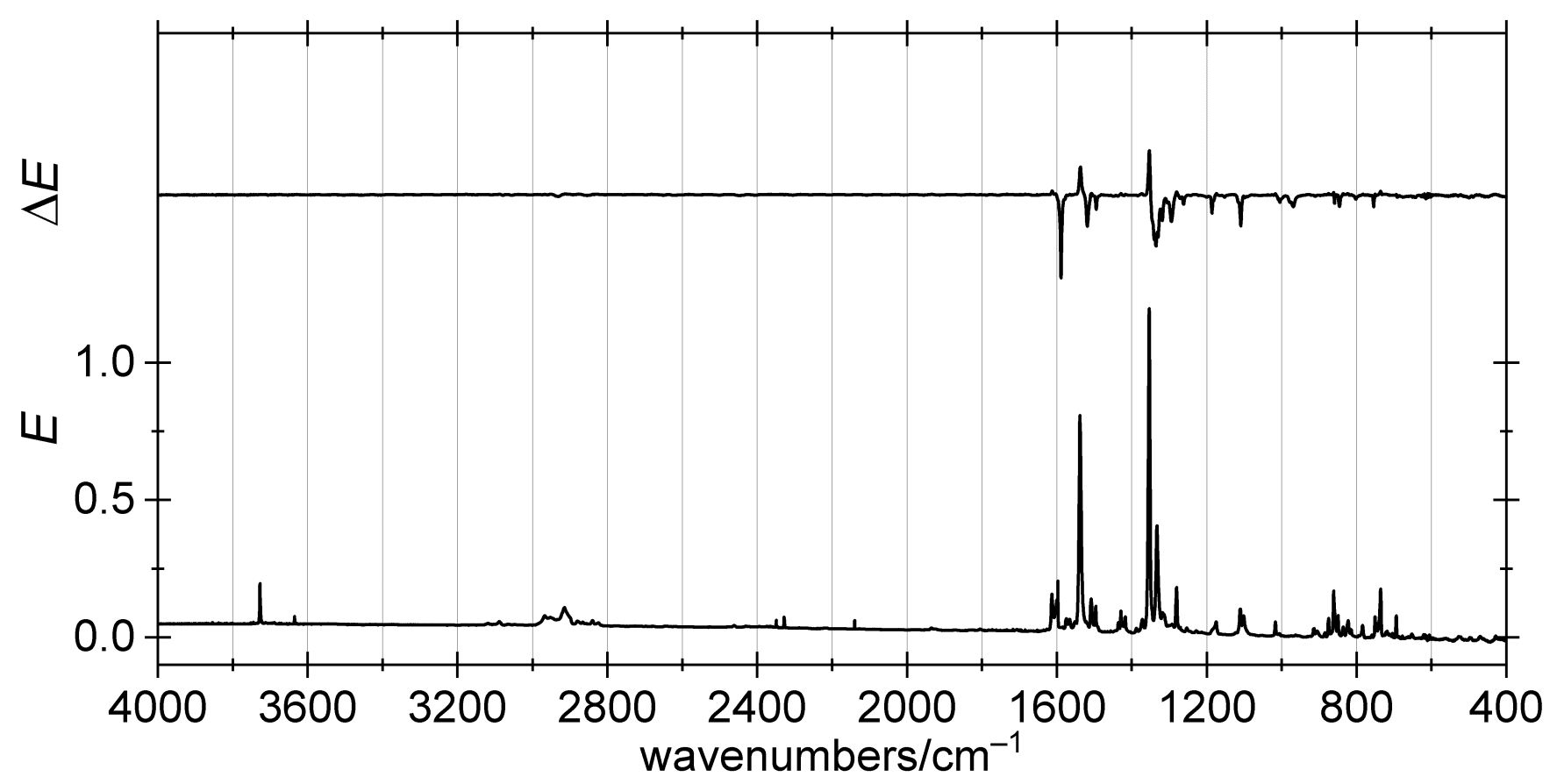

Figure S13: Matrix-isolated $p$-NBA dithiane in $\mathrm{N}_{2}+1 \% \mathrm{O}_{2}$ at $3 \mathrm{~K}$ after $60 \mathrm{~s}$ irradiation at $313 \mathrm{~nm}$ and $60 \mathrm{~s}$ irradiation at $436 \mathrm{~nm}$; difference trace (before/after irradiation at $436 \mathrm{~nm}$ ) on top.

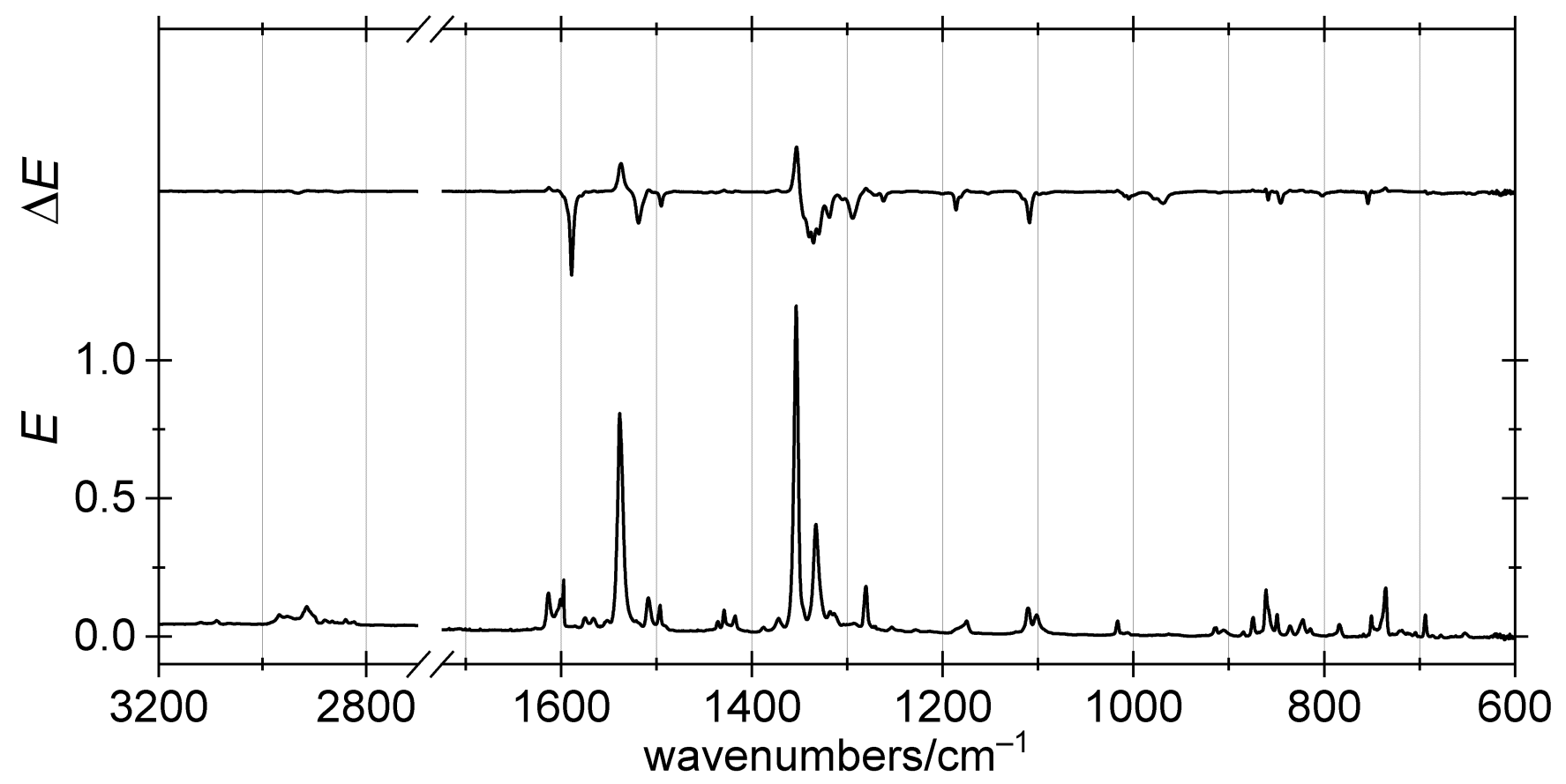

Figure S14: Matrix-isolated $p$-NBA dithiane in $\mathrm{N}_{2}+1 \% \mathrm{O}_{2}$ at $3 \mathrm{~K}$ after $60 \mathrm{~s}$ irradiation at $313 \mathrm{~nm}$ and $60 \mathrm{~s}$ irradiation at $436 \mathrm{~nm}$ (detail); difference trace (before/after irradiation at $436 \mathrm{~nm}$ ) on top. 


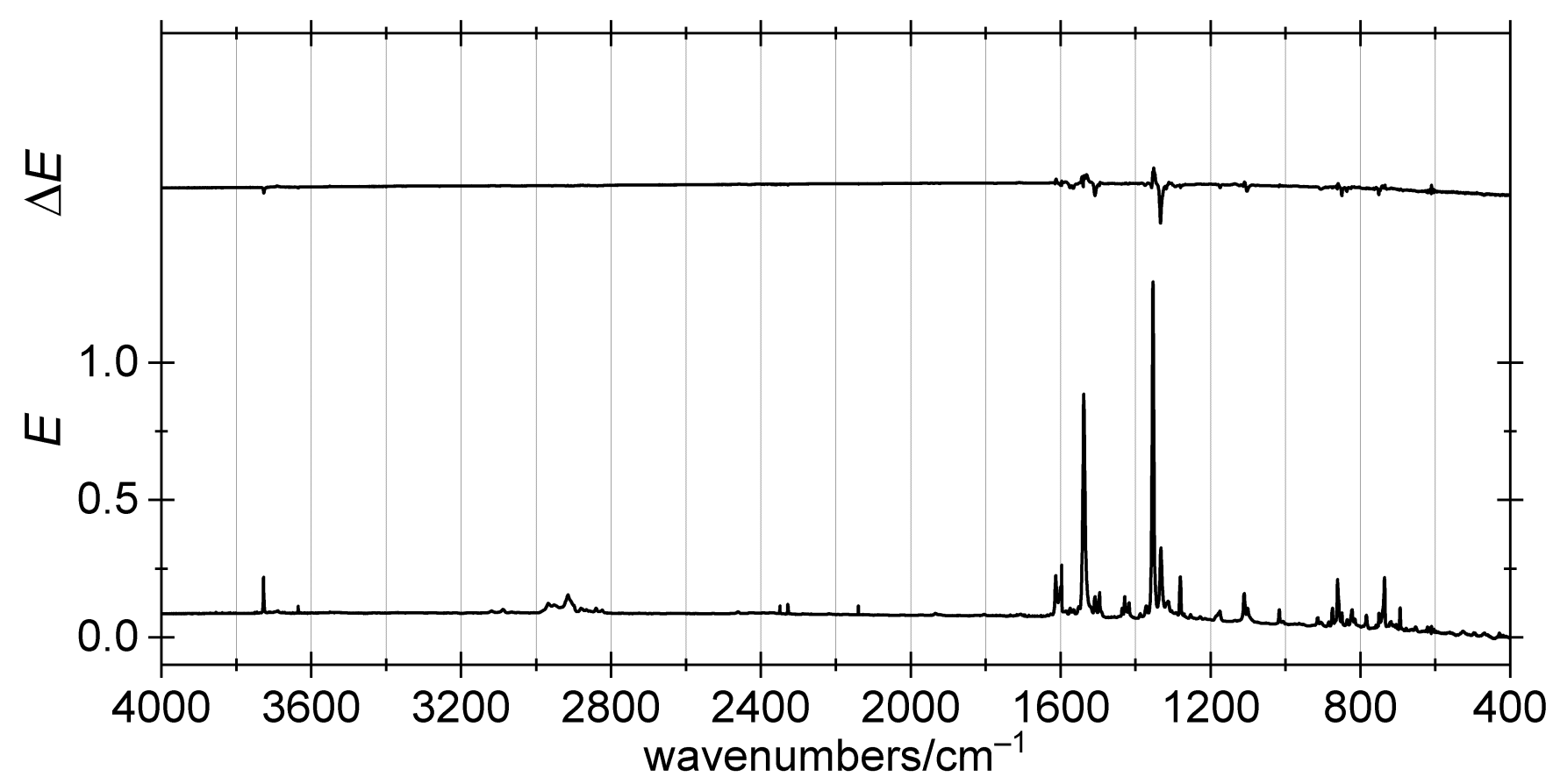

Figure S15: Matrix-isolated $p$-NBA dithiane in $\mathrm{N}_{2}+1 \% \mathrm{O}_{2}$ at $3 \mathrm{~K}$ after $60 \mathrm{~s}$ irradiation at $313 \mathrm{~nm}, 60 \mathrm{~s}$ irradiation at $436 \mathrm{~nm}$, and subsequent annealing to $27 \mathrm{~K}$; difference trace (before/after annealing to $27 \mathrm{~K}$ ) on top.

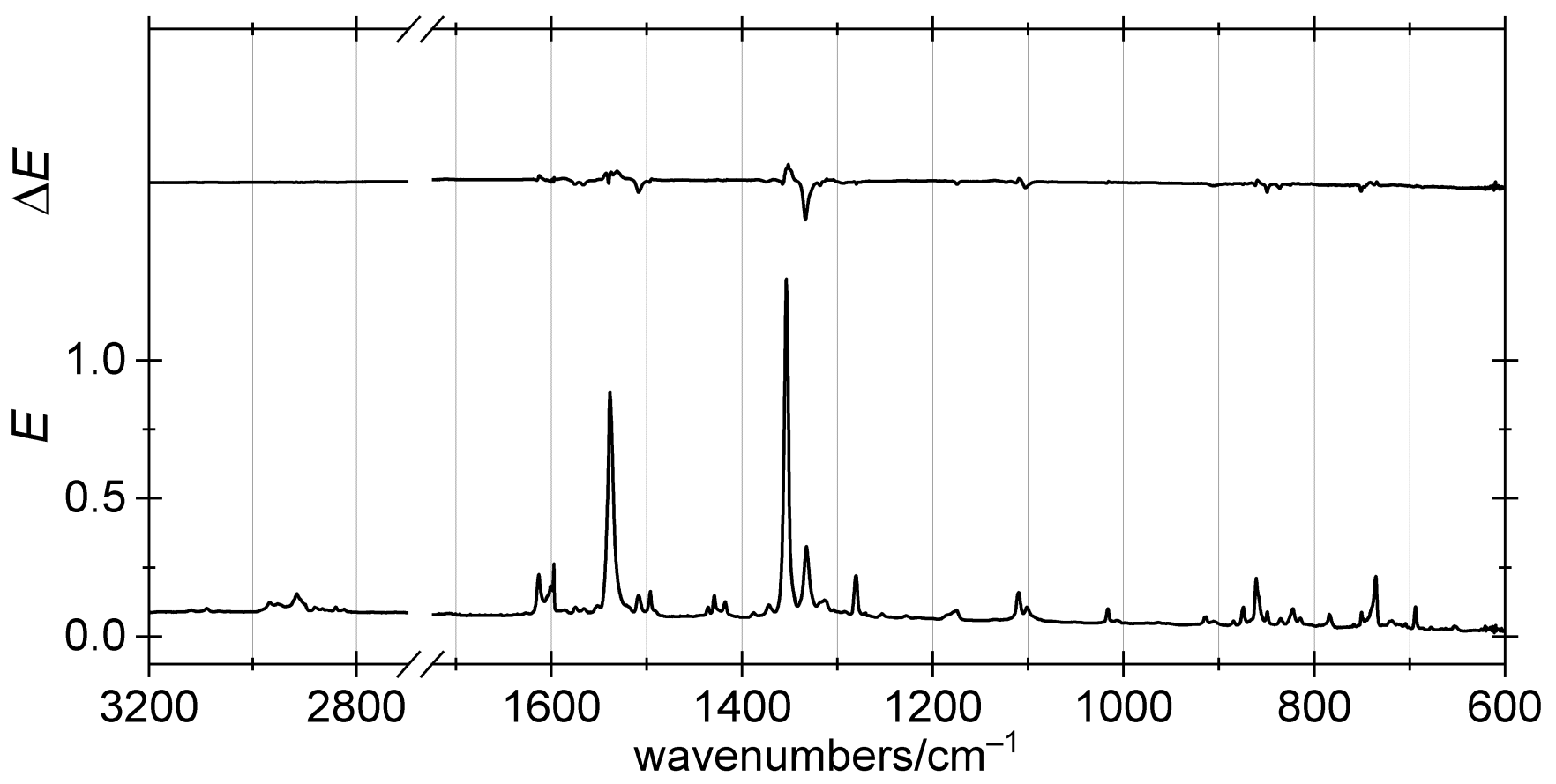

Figure S16: Matrix-isolated $p$-NBA dithiane in $\mathrm{N}_{2}+1 \% \mathrm{O}_{2}$ at $3 \mathrm{~K}$ after $60 \mathrm{~s}$ irradiation at $313 \mathrm{~nm}, 60 \mathrm{~s}$ irradiation at $436 \mathrm{~nm}$, and subsequent annealing to $27 \mathrm{~K}$ (detail); difference trace (before/after annealing to $27 \mathrm{~K}$ ) on top. 


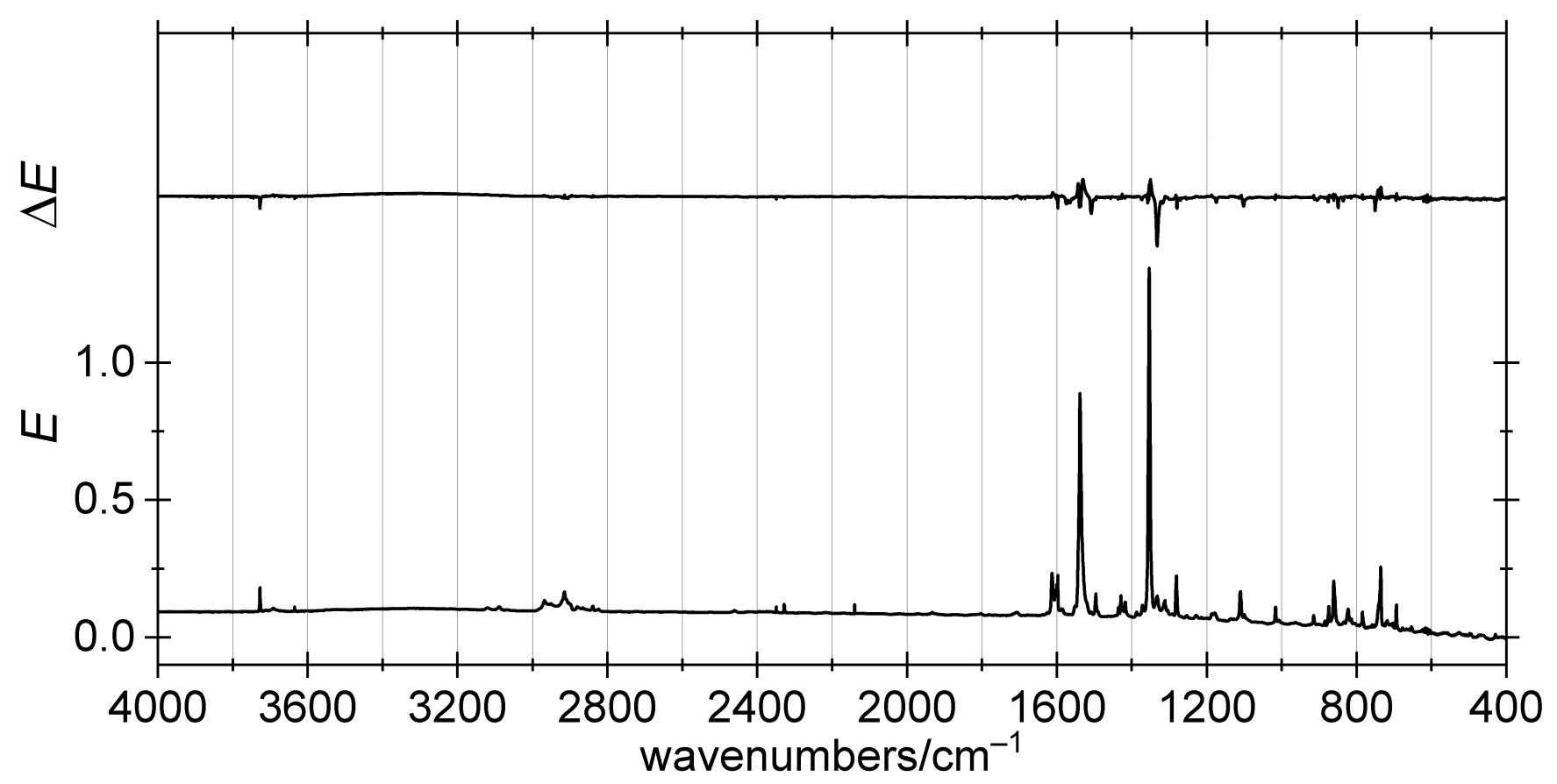

Figure S17: Matrix-isolated $p$-NBA dithiane in $\mathrm{N}_{2}+1 \% \mathrm{O}_{2}$ at $3 \mathrm{~K}$ after $60 \mathrm{~s}$ irradiation at $313 \mathrm{~nm}, 60 \mathrm{~s}$ irradiation at $436 \mathrm{~nm}$, and subsequent annealing to 27 , then $33 \mathrm{~K}$; difference trace (before/after annealing to $33 \mathrm{~K}$ ) on top.

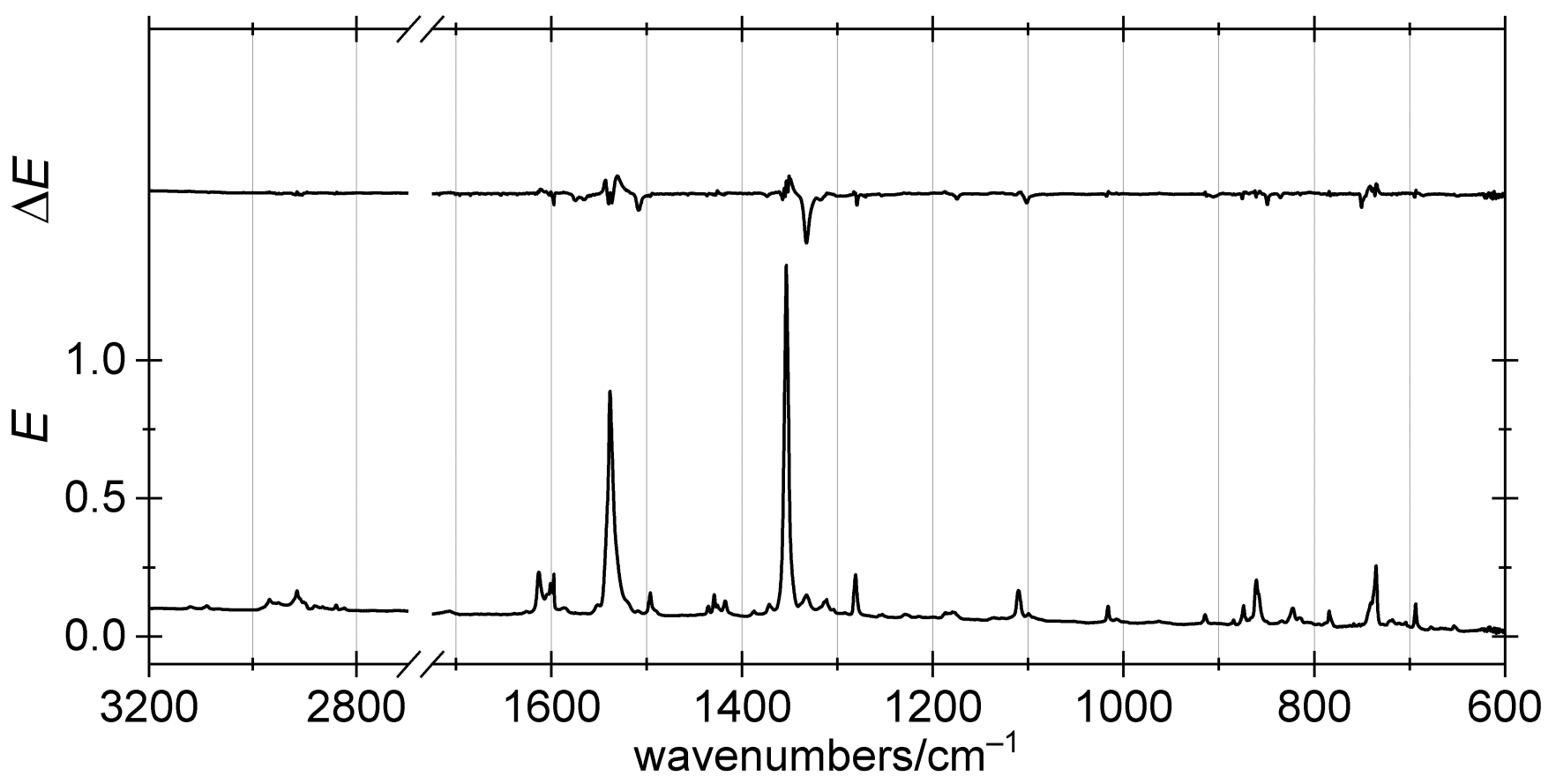

Figure S18: Matrix-isolated $p$-NBA dithiane in $\mathrm{N}_{2}+1 \% \mathrm{O}_{2}$ at $3 \mathrm{~K}$ after $60 \mathrm{~s}$ irradiation at $313 \mathrm{~nm}, 60 \mathrm{~s}$ irradiation at $436 \mathrm{~nm}$, and subsequent annealing to 27, then $33 \mathrm{~K}$ (detail); difference trace (before/after annealing to $33 \mathrm{~K}$ ) on top. 
1.3. IR spectra of $d_{1}-p$-NBA dithiane in $\mathrm{N}_{2}$

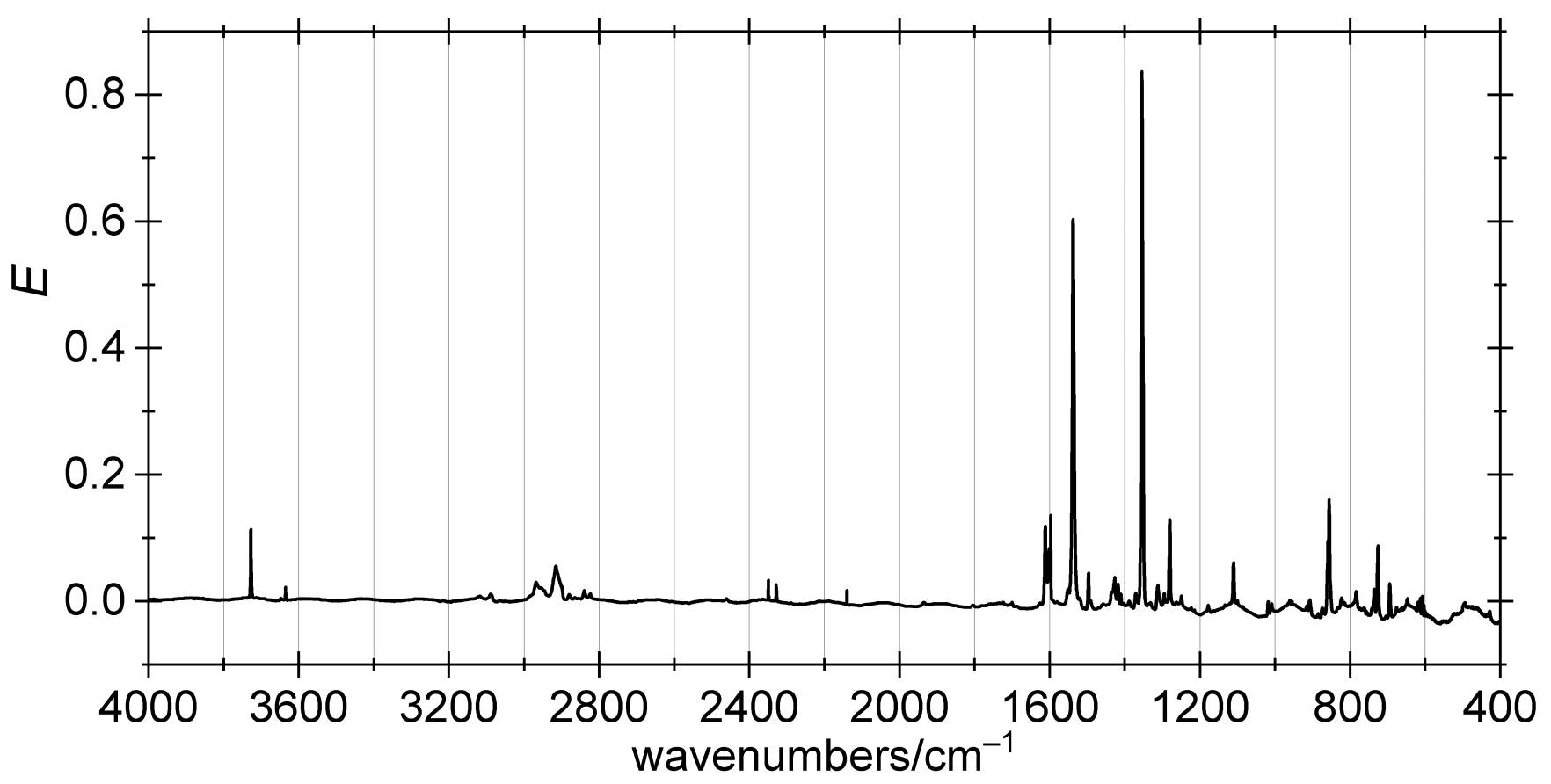

Figure S19: Matrix-isolated $d_{1}-p$-NBA dithiane in $\mathrm{N}_{2}$ at $3 \mathrm{~K}$.

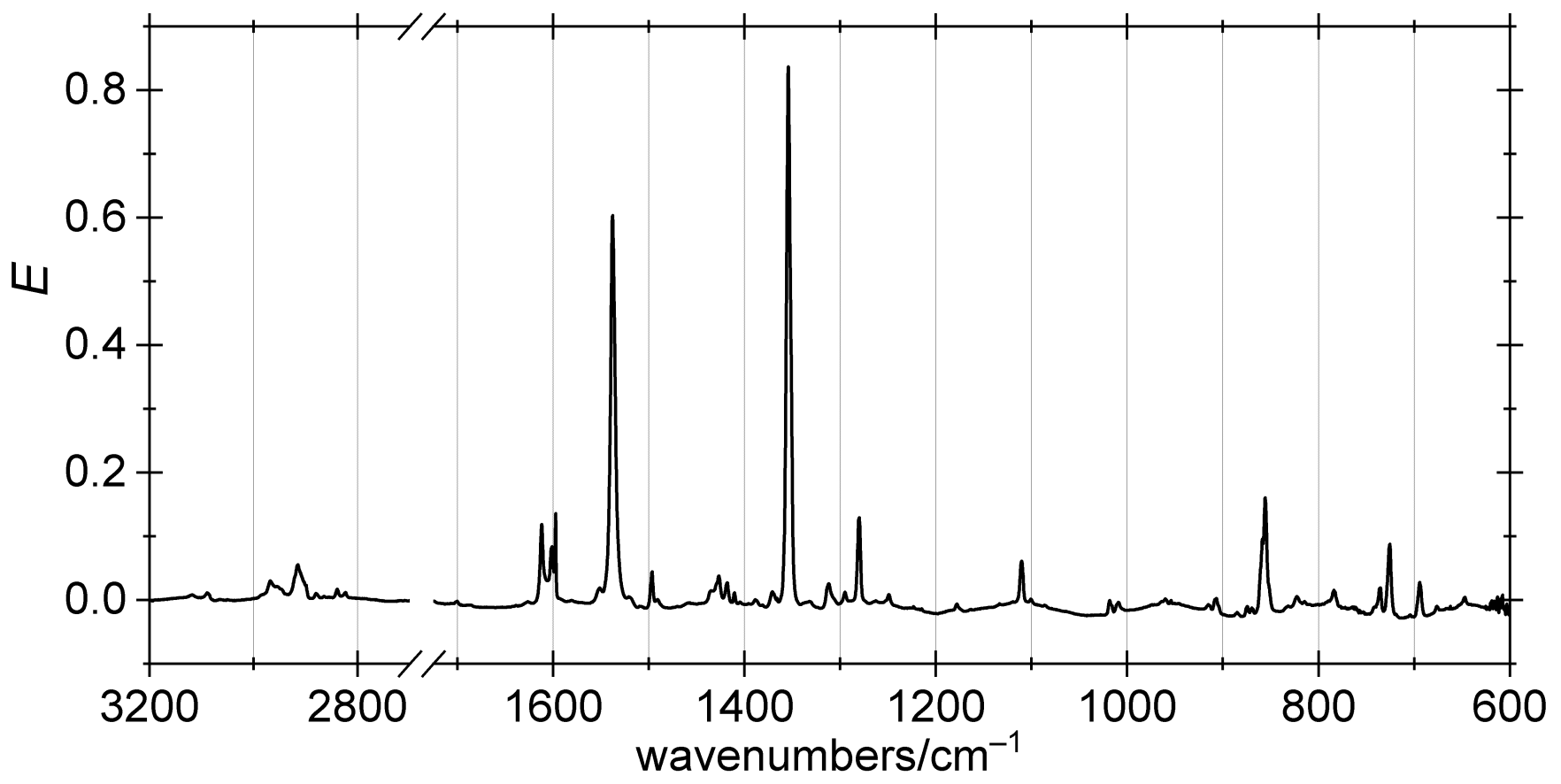

Figure S20: Matrix-isolated $d_{1}-p$-NBA dithiane in $\mathrm{N}_{2}$ at $3 \mathrm{~K}$ (detail). 


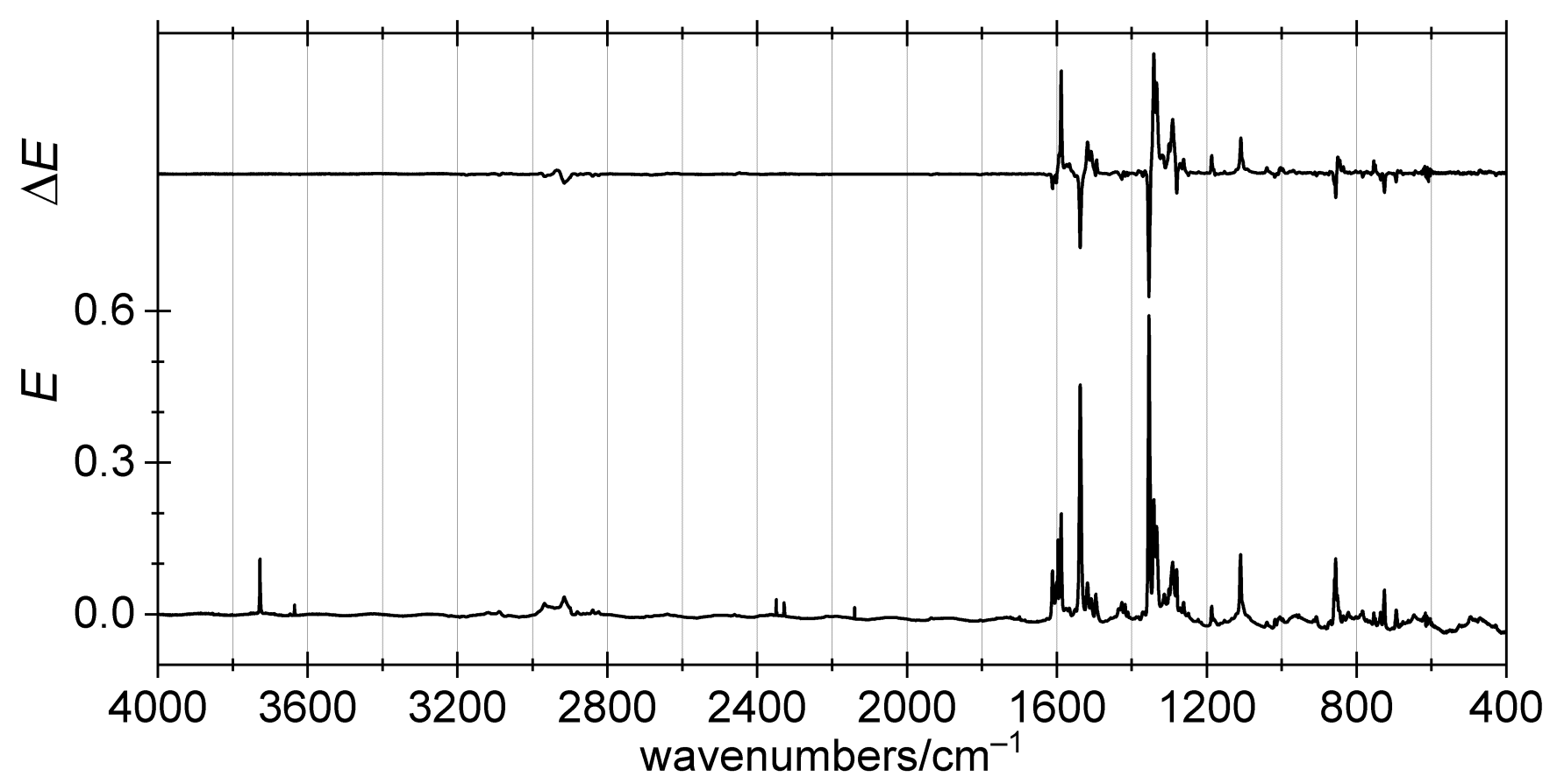

Figure S21: Matrix-isolated $d_{1}-p$-NBA dithiane in $\mathrm{N}_{2}$ at $3 \mathrm{~K}$ after $30 \mathrm{~s}$ irradiation at $313 \mathrm{~nm}$; difference trace (before/after irradiation at $313 \mathrm{~nm}$ ) on top.

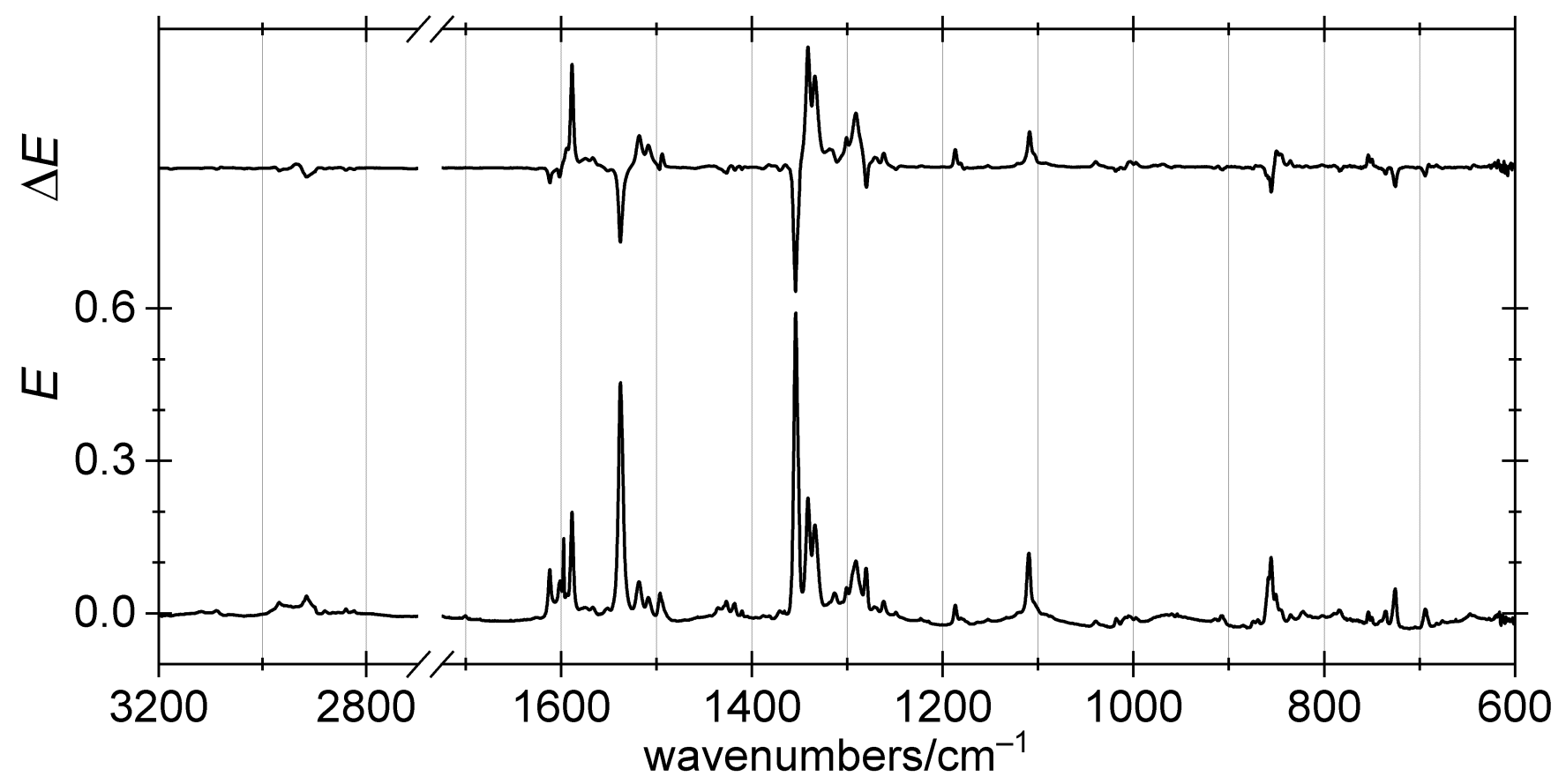

Figure S22: Matrix-isolated $d_{1}-p$-NBA dithiane in $\mathrm{N}_{2}$ at $3 \mathrm{~K}$ after $30 \mathrm{~s}$ irradiation at $313 \mathrm{~nm}$ (detail); difference trace (before/after irradiation at $313 \mathrm{~nm}$ ) on top. 


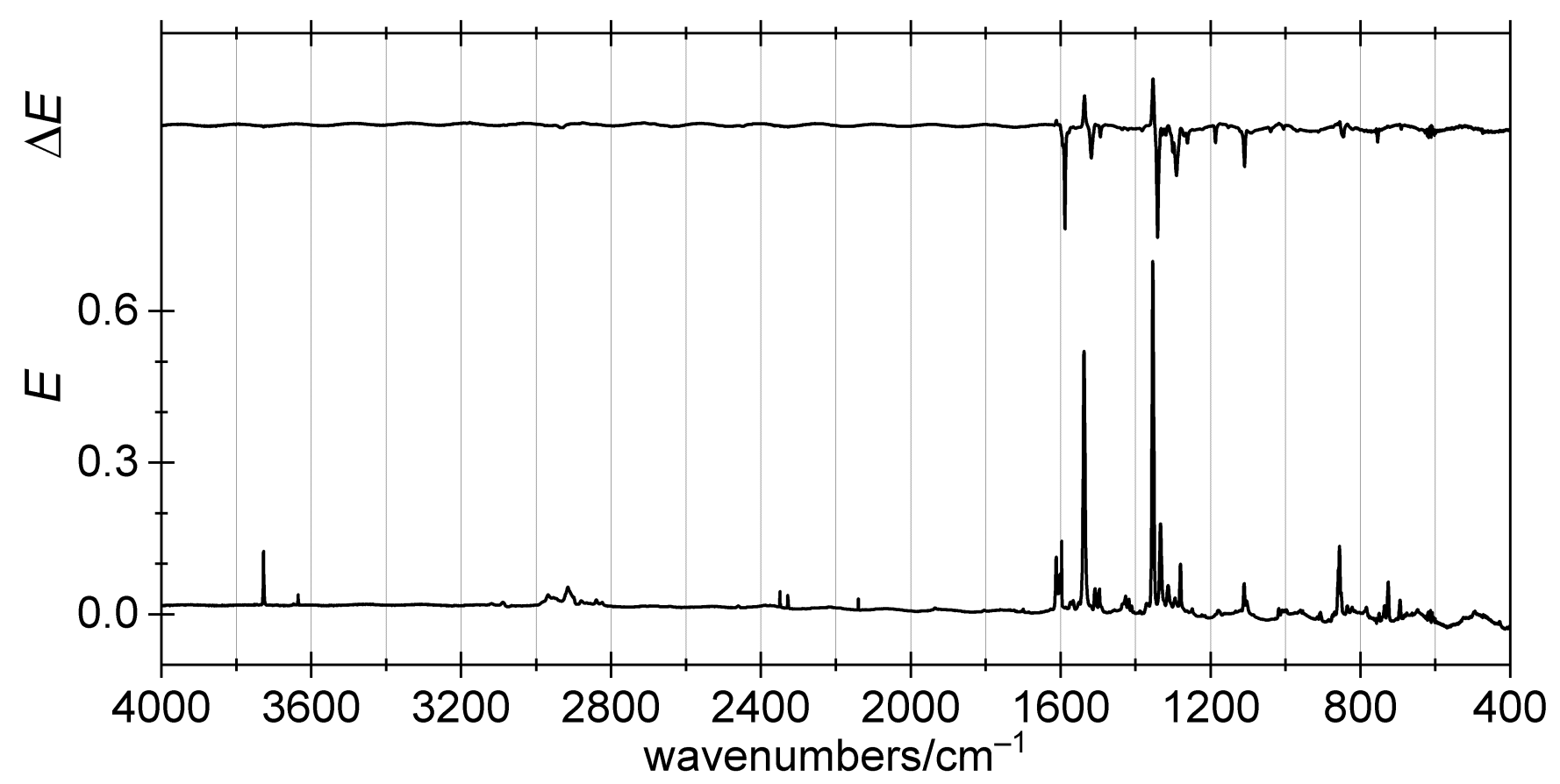

Figure S23: Matrix-isolated $d_{1}-p$-NBA dithiane in $\mathrm{N}_{2}$ at $3 \mathrm{~K}$ after $30 \mathrm{~s}$ irradiation at $313 \mathrm{~nm}$ and $60 \mathrm{~s}$ irradiation at $436 \mathrm{~nm}$; difference trace (before/after irradiation at $436 \mathrm{~nm}$ ) on top.

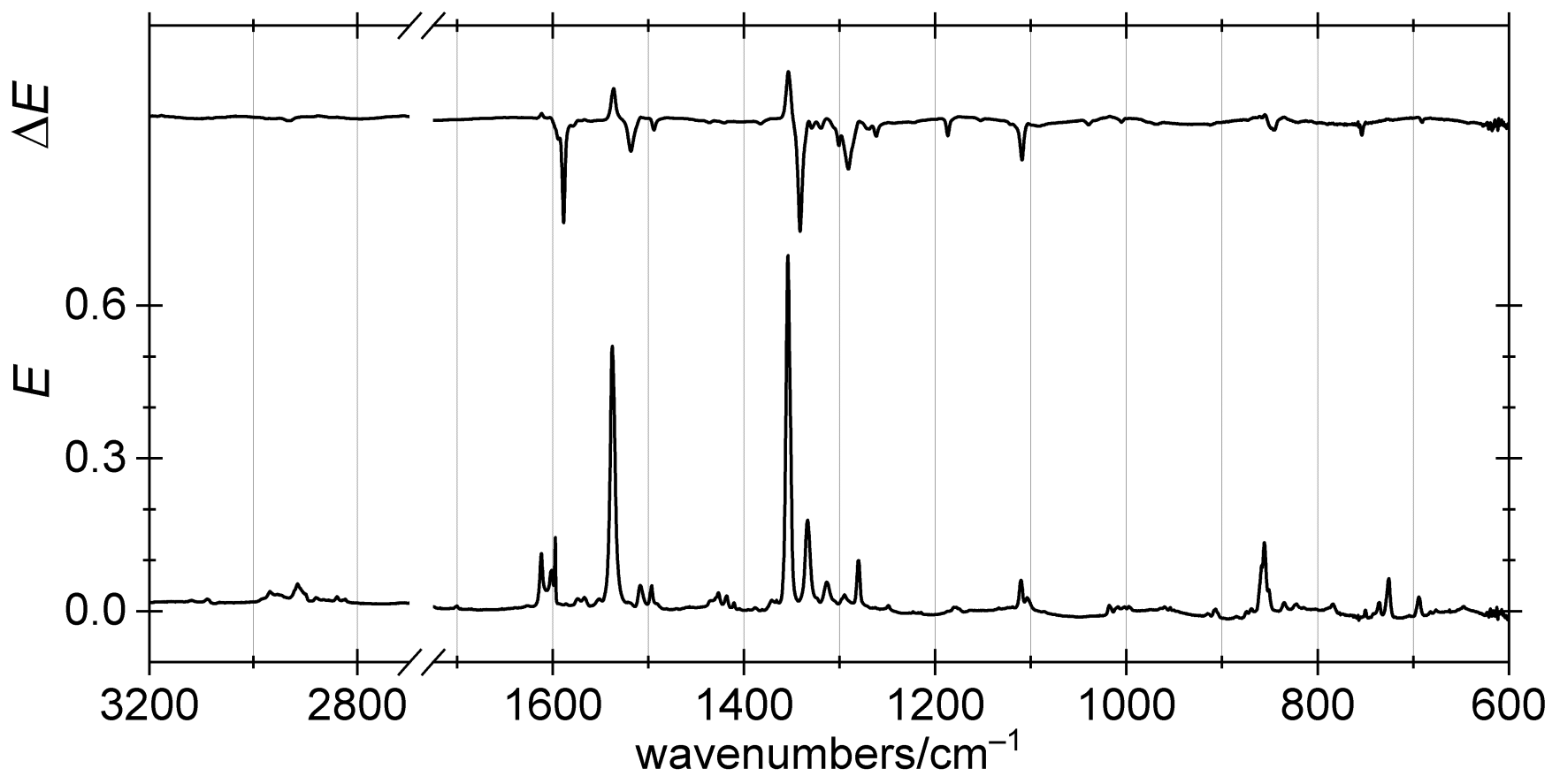

Figure S24: Matrix-isolated $d_{1}-p$-NBA dithiane in $\mathrm{N}_{2}$ at $3 \mathrm{~K}$ after $30 \mathrm{~s}$ irradiation at $313 \mathrm{~nm}$ and $60 \mathrm{~s}$ irradiation at $436 \mathrm{~nm}$ (detail); difference trace (before/after irradiation at $436 \mathrm{~nm}$ ) on top. 


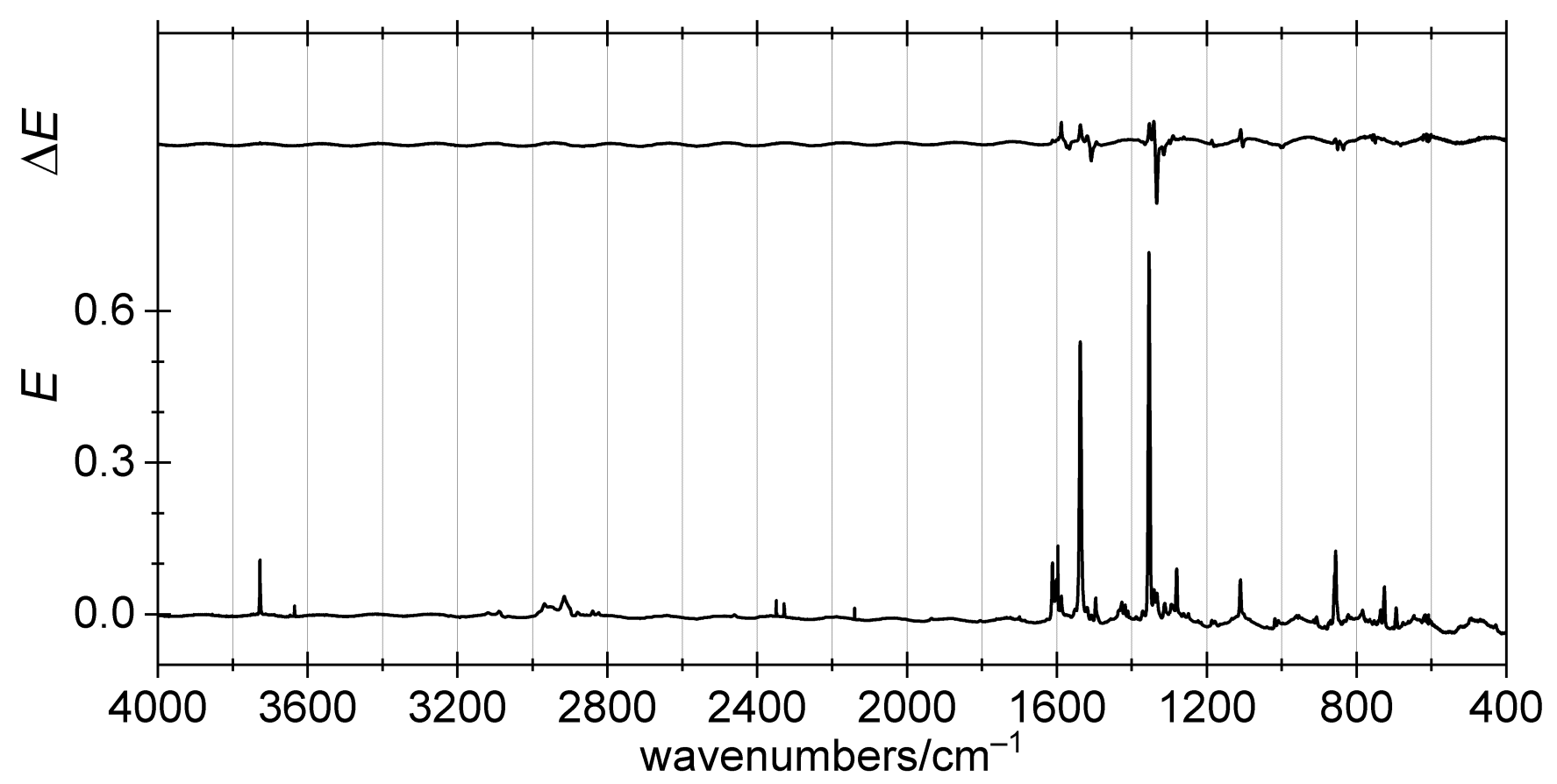

Figure S25: Matrix-isolated $d_{1}-p$-NBA dithiane in $\mathrm{N}_{2}$ at $3 \mathrm{~K}$ after $30 \mathrm{~s}$ irradiation at $313 \mathrm{~nm}, 60 \mathrm{~s}$ irradiation at $436 \mathrm{~nm}$, and $15 \mathrm{~min}$ irradiation at $546 \mathrm{~nm}$; difference trace (before/after irradiation at $546 \mathrm{~nm}$ ) on top.

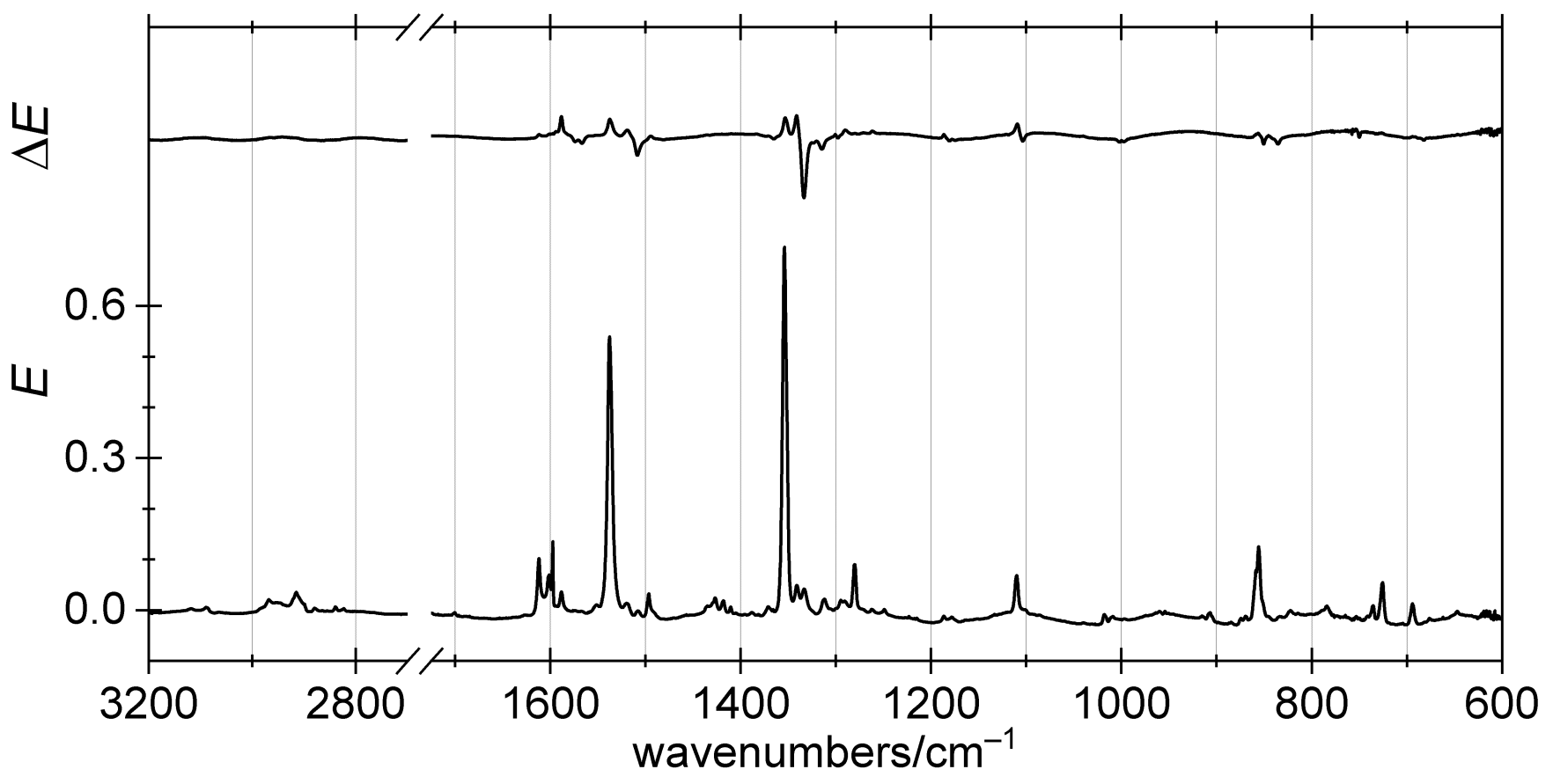

Figure S26: Matrix-isolated $d_{1}-p$-NBA dithiane in $\mathrm{N}_{2}$ at $3 \mathrm{~K}$ after $30 \mathrm{~s}$ irradiation at $313 \mathrm{~nm}, 60 \mathrm{~s}$ irradiation at $436 \mathrm{~nm}$, and $15 \mathrm{~min}$ irradiation at $546 \mathrm{~nm}$ (detail); difference trace (before/after irradiation at $546 \mathrm{~nm}$ ) on top. 
1.4. UV/Vis spectra of $p$-NBA dithiane in $\mathrm{N}_{2}$

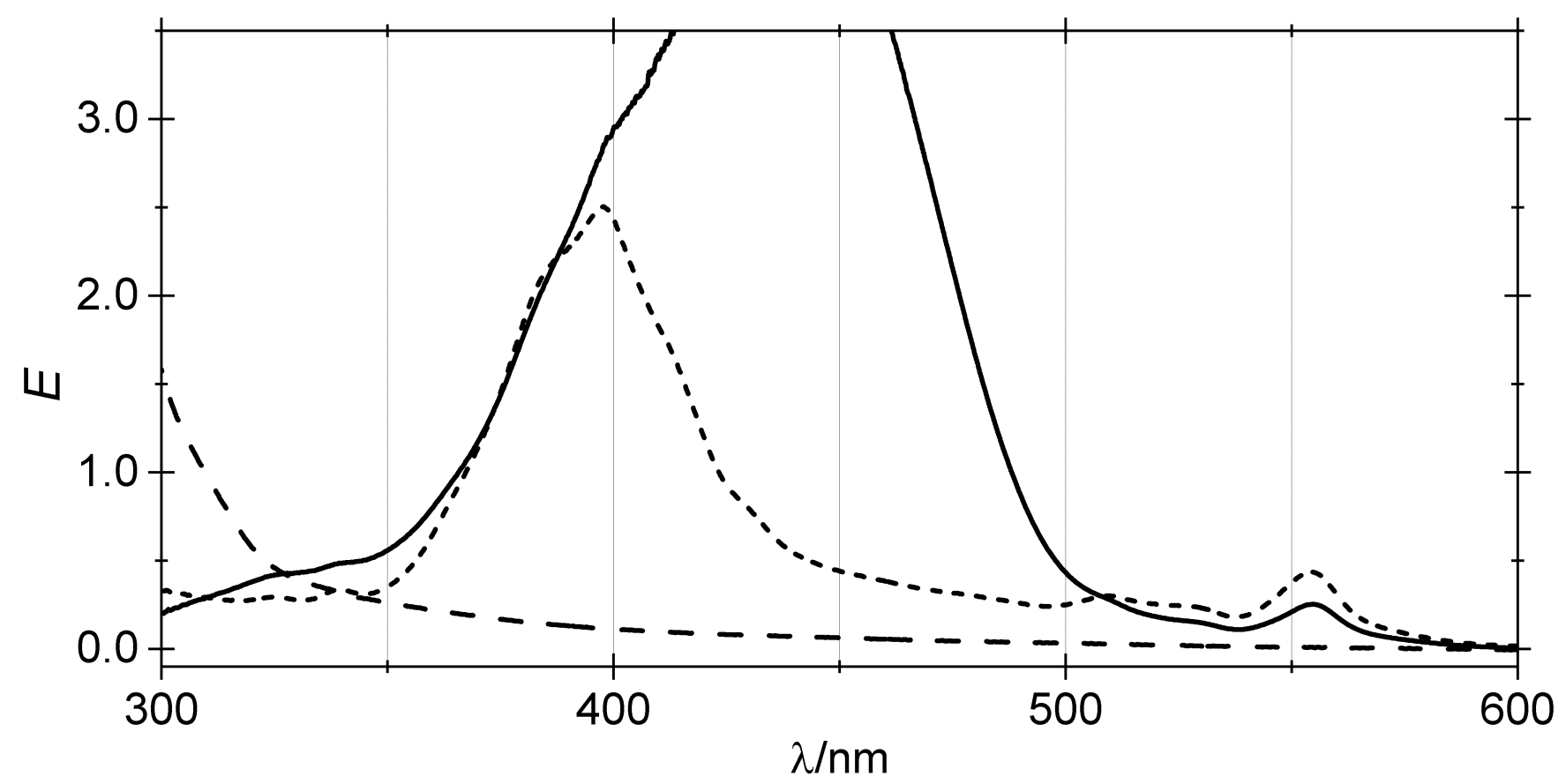

Figure S27: Matrix-isolated $p$-NBA dithiane in $\mathrm{N}_{2}$ at $3 \mathrm{~K}$ after deposition (dashed trace), after $60 \mathrm{~s}$ irradiation at $313 \mathrm{~nm}$ (solid trace), and after $90 \mathrm{~s}$ irradiation at $436 \mathrm{~nm}$ (short-dashed trace).

1.5. UV/Vis spectra of $d_{1}-p$-NBA dithiane in $\mathrm{N}_{2}$

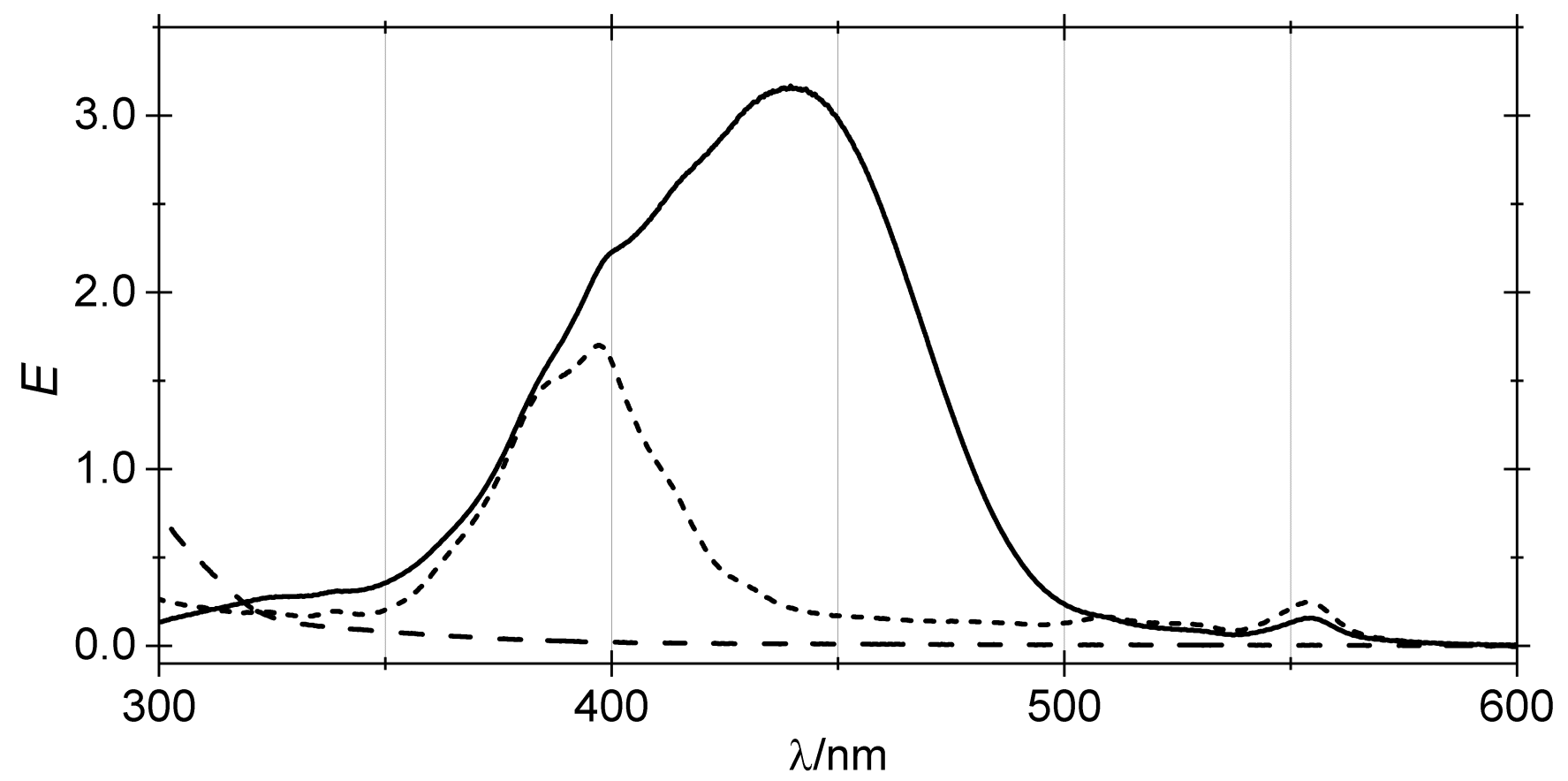

Figure S28: Matrix-isolated $d_{1}-p$-NBA dithiane in $\mathrm{N}_{2}$ at $3 \mathrm{~K}$ after deposition (dashed trace), after $30 \mathrm{~s}$ irradiation at $313 \mathrm{~nm}$ (solid trace), and after $60 \mathrm{~s}$ irradiation at $436 \mathrm{~nm}$ (short-dashed trace). 
2. Matrix isolation experiments on $p$-nitrobenzaldehyde dimethyl bisthioacetal ( $p$-NBA-DMTA)

2.1. IR spectra of $p$-NBA-DMTA in $\mathrm{N}_{2}$

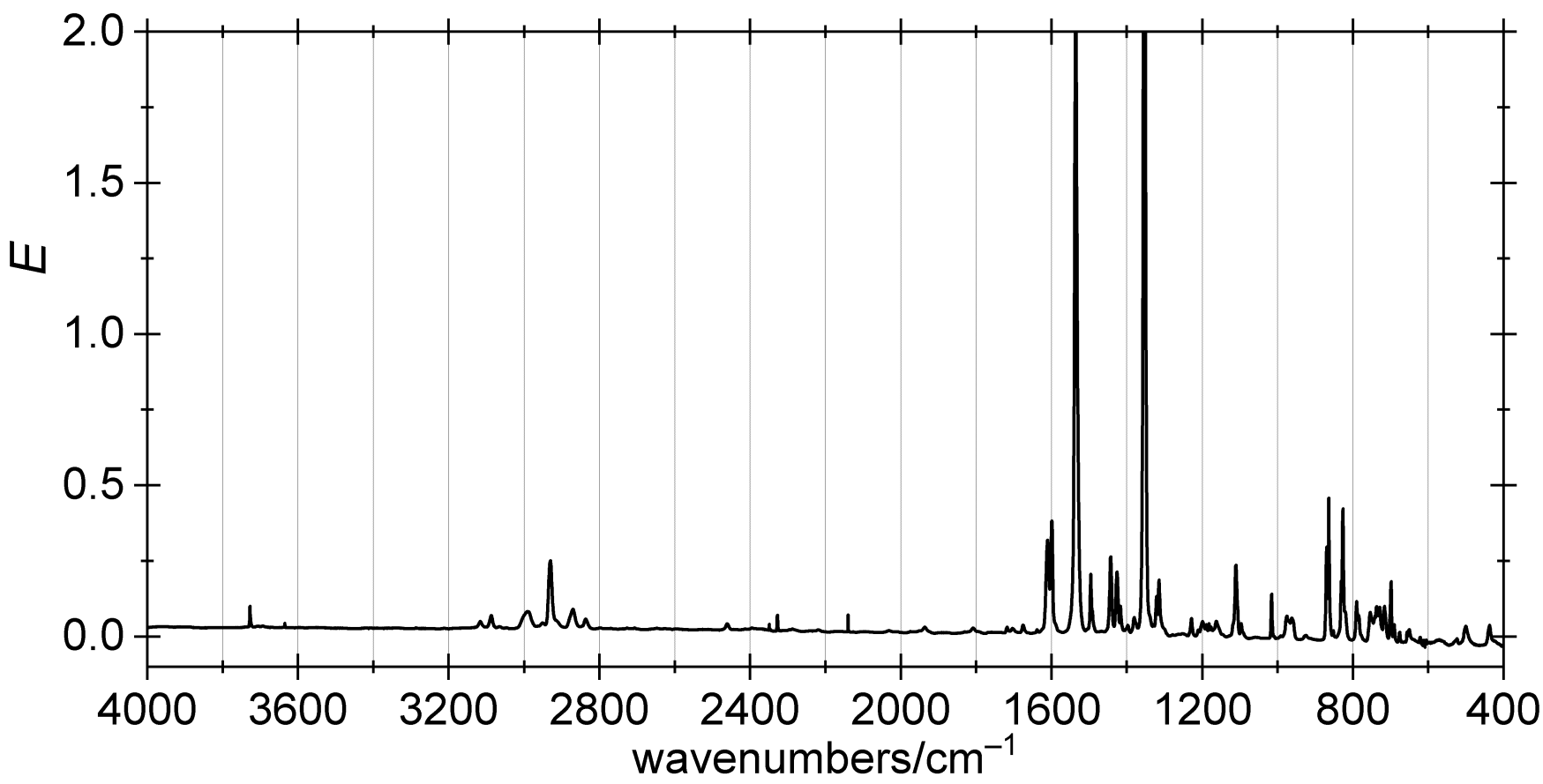

Figure S29: Matrix-isolated $p$-NBA-DMTA in $\mathrm{N}_{2}$ at $3 \mathrm{~K}$.

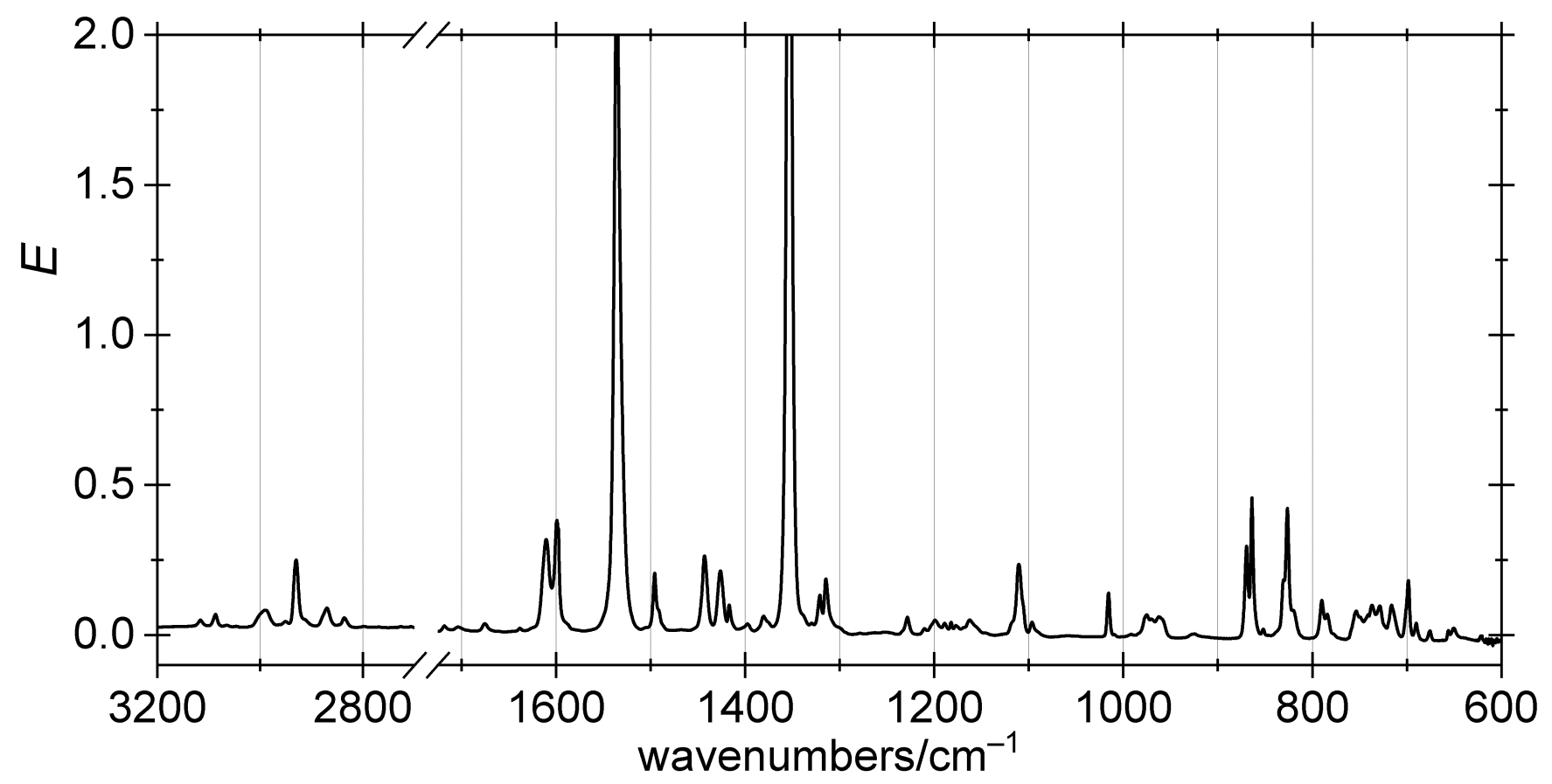

Figure S30: Matrix-isolated $p$-NBA-DMTA in $\mathrm{N}_{2}$ at $3 \mathrm{~K}$ (detail). 


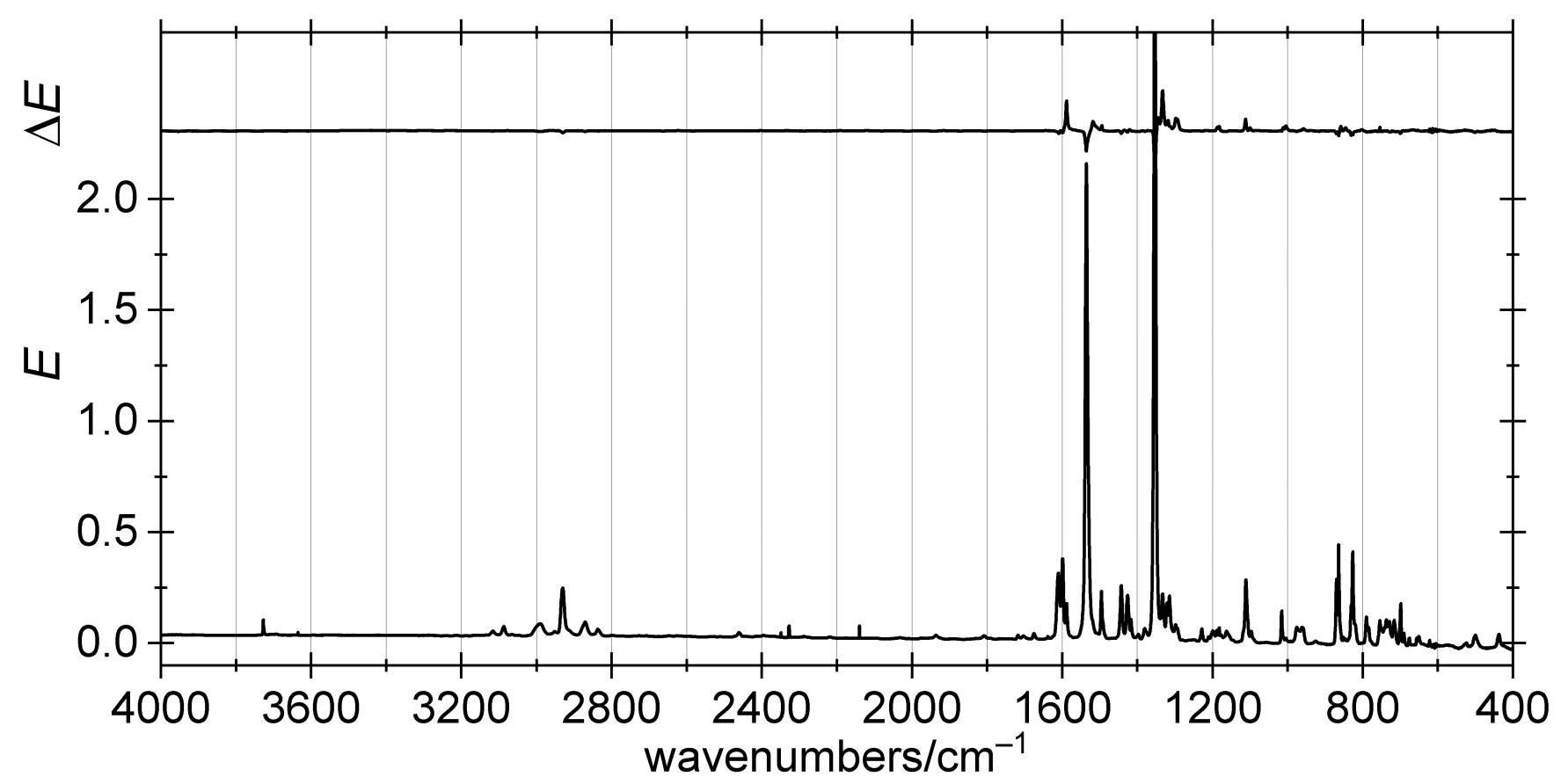

Figure S31: Matrix-isolated $p$-NBA-DMTA in $\mathrm{N}_{2}$ at $3 \mathrm{~K}$ after $60 \mathrm{~s}$ irradiation at $313 \mathrm{~nm}$; difference trace (before/after irradiation at $313 \mathrm{~nm}$ ) on top.

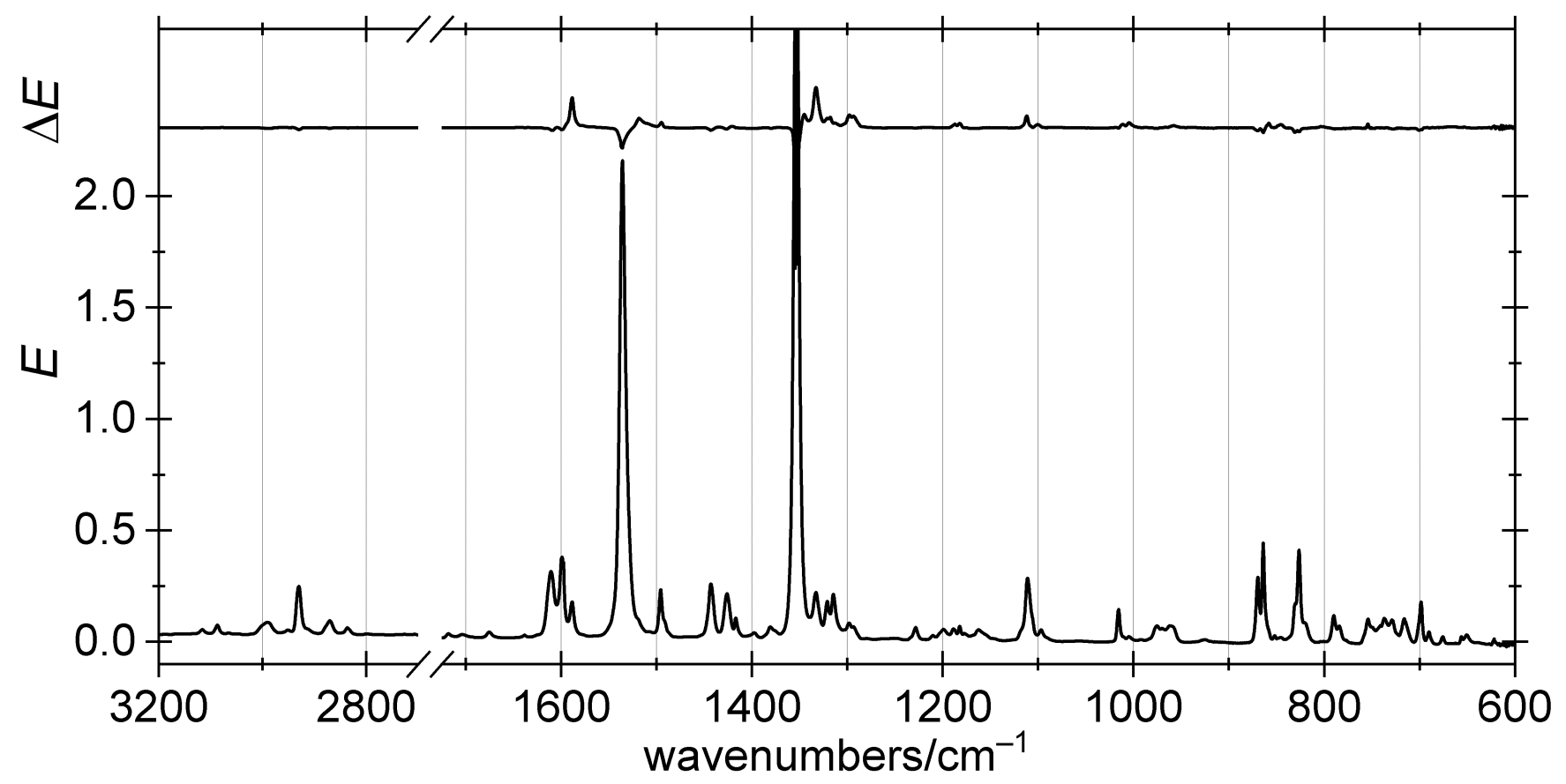

Figure S32: Matrix-isolated $p$-NBA-DMTA in $\mathrm{N}_{2}$ at $3 \mathrm{~K}$ after $60 \mathrm{~s}$ irradiation at $313 \mathrm{~nm}$ (detail); difference trace (before/after irradiation at $313 \mathrm{~nm}$ ) on top. 
2.2. UV/Vis spectra of $p$-NBA-DMTA in $\mathrm{N}_{2}$

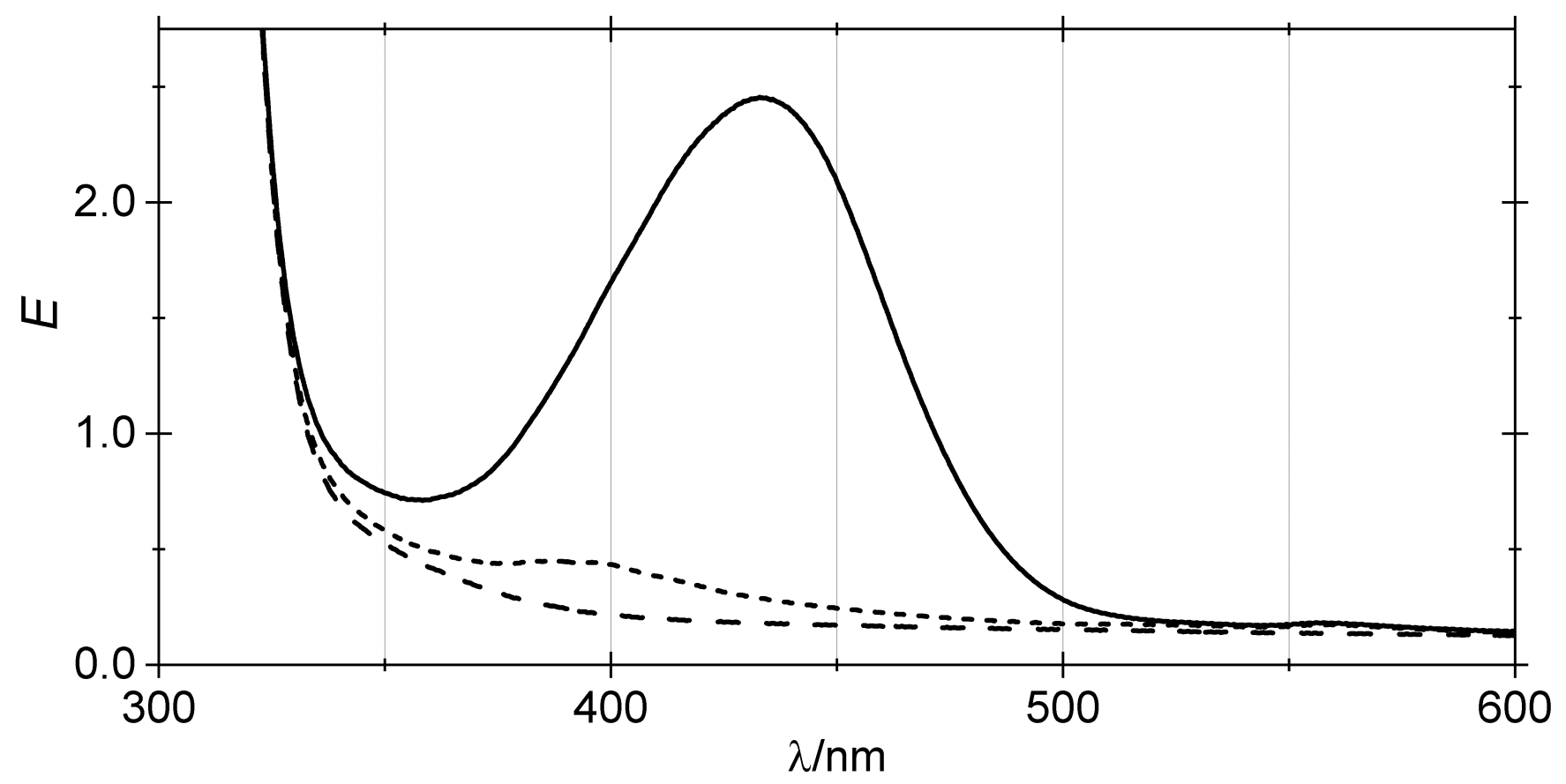

Figure S33: Matrix-isolated $p$-NBA-DMTA in $\mathrm{N}_{2}$ at $3 \mathrm{~K}$ after deposition (dashed trace), after $60 \mathrm{~s}$ irradiation at $313 \mathrm{~nm}$ (solid trace), and after $90 \mathrm{~s}$ irradiation at $436 \mathrm{~nm}$ (short-dashed trace). 
3. Matrix isolation experiments on $o$-nitrobenzaldehyde (o-NBA) dithiane

3.1. IR spectra of $o$-NBA dithiane in $\mathrm{N}_{2}$

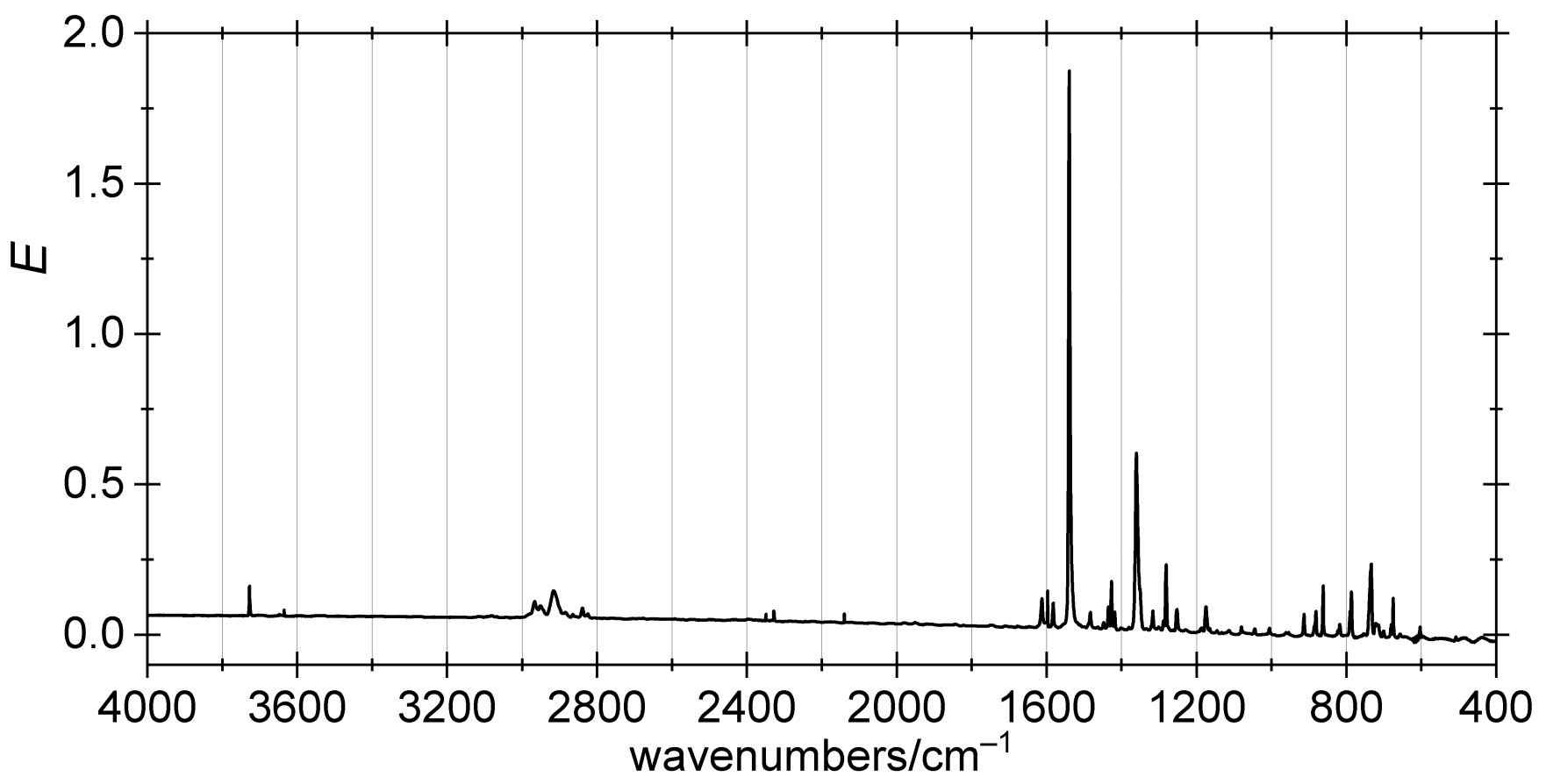

Figure S34: Matrix-isolated $o$-NBA dithiane in $\mathrm{N}_{2}$ at $3 \mathrm{~K}$.

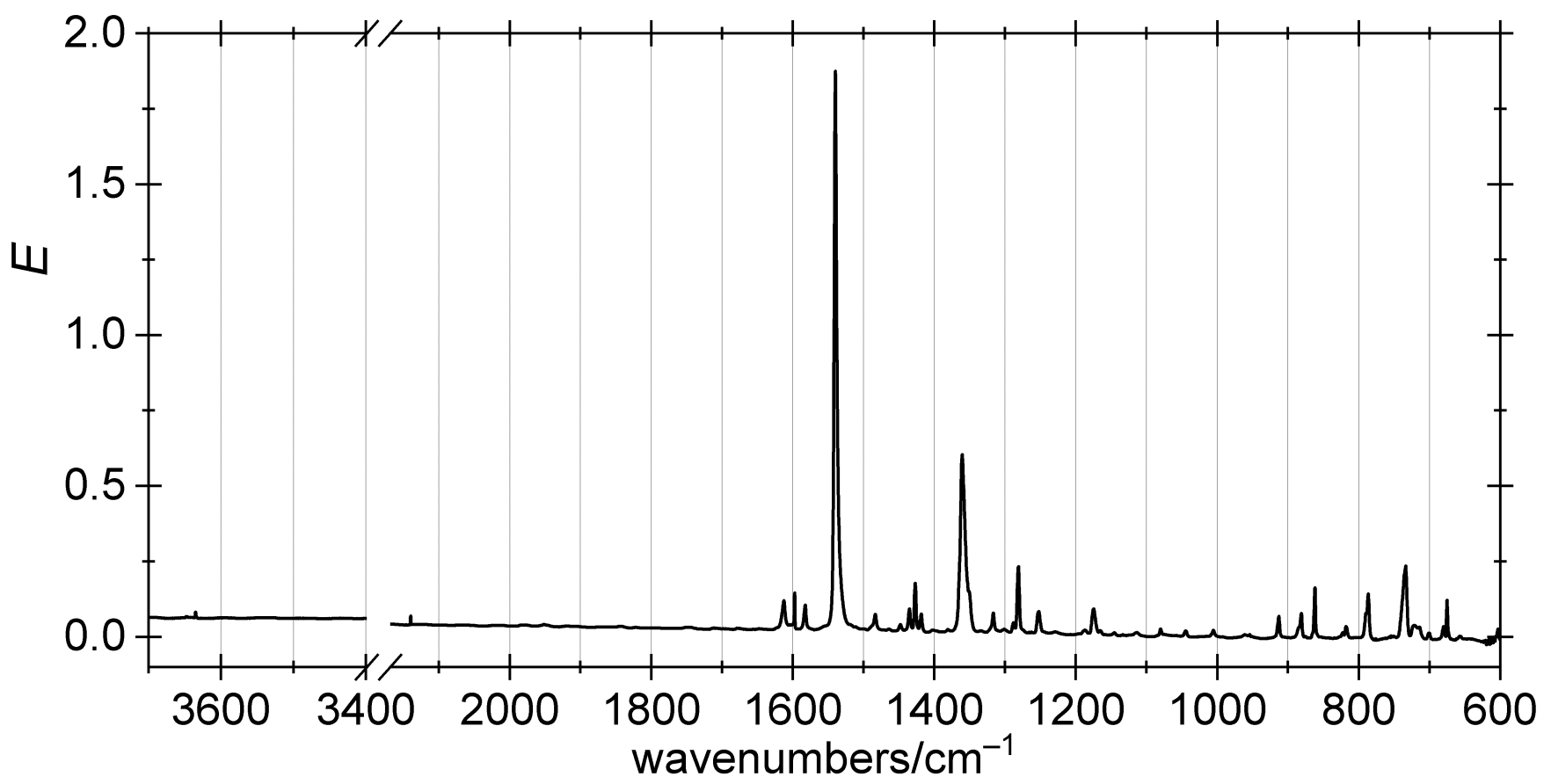

Figure S35: Matrix-isolated $o$-NBA dithiane in $\mathrm{N}_{2}$ at $3 \mathrm{~K}$ (detail). 


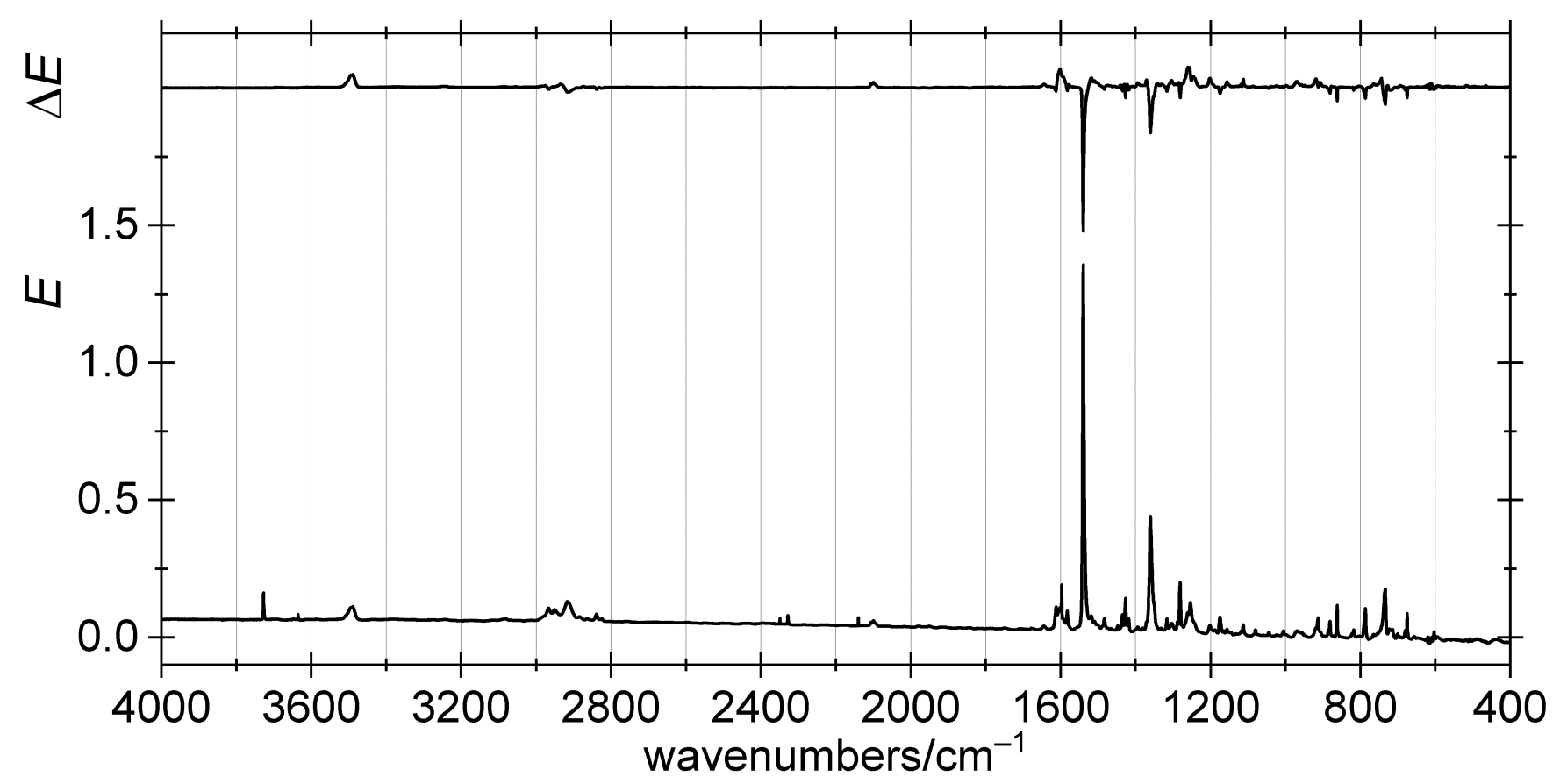

Figure S36: Matrix-isolated $o$-NBA dithiane in $\mathrm{N}_{2}$ at $3 \mathrm{~K}$ after $60 \mathrm{~s}$ irradiation at $313 \mathrm{~nm}$; difference trace (before/after irradiation at $313 \mathrm{~nm}$ ) on top.

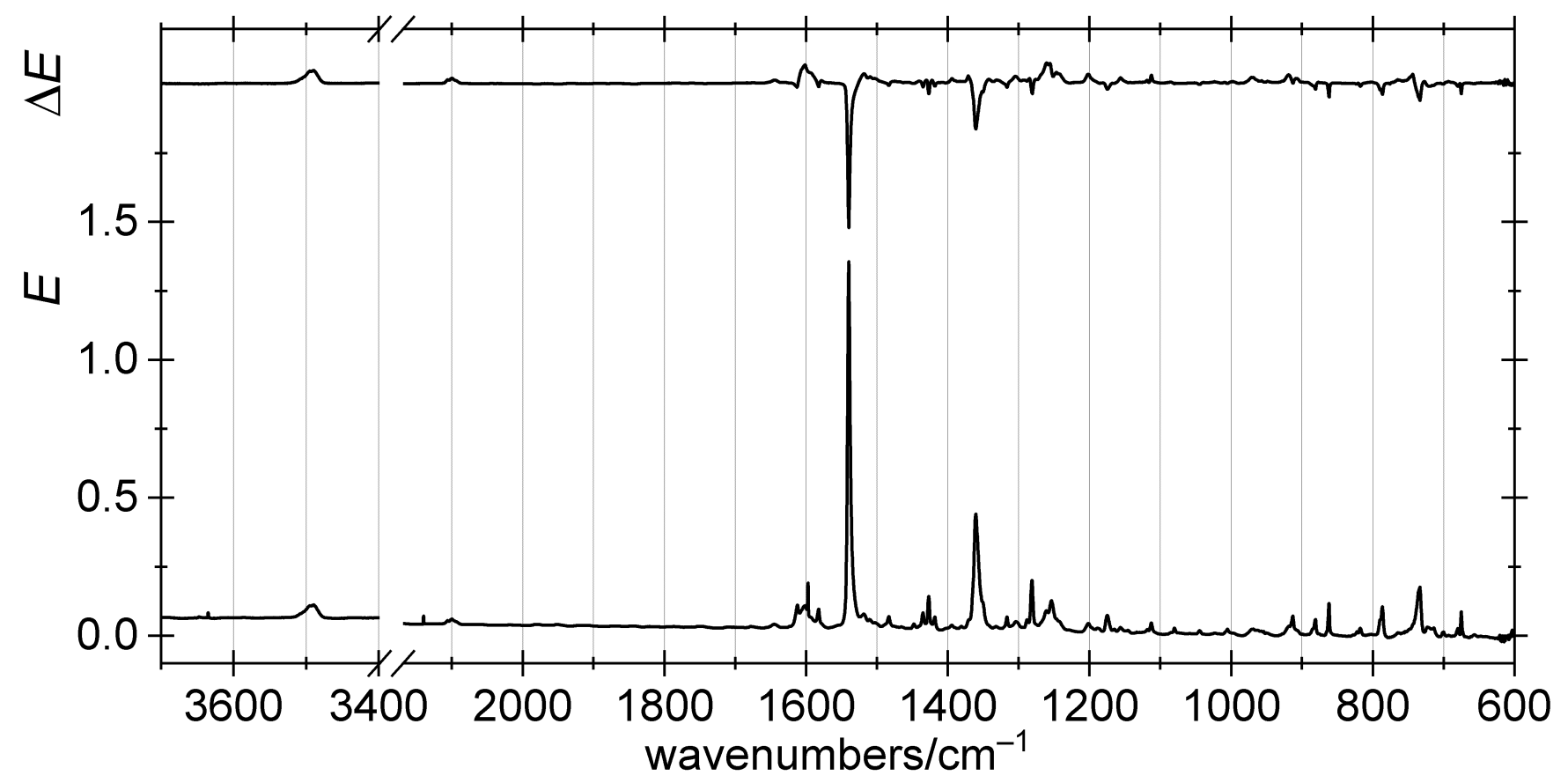

Figure S37: Matrix-isolated $o$-NBA dithiane in $\mathrm{N}_{2}$ at $3 \mathrm{~K}$ after $60 \mathrm{~s}$ irradiation at $313 \mathrm{~nm}$ (detail); difference trace (before/after irradiation at $313 \mathrm{~nm}$ ) on top. 


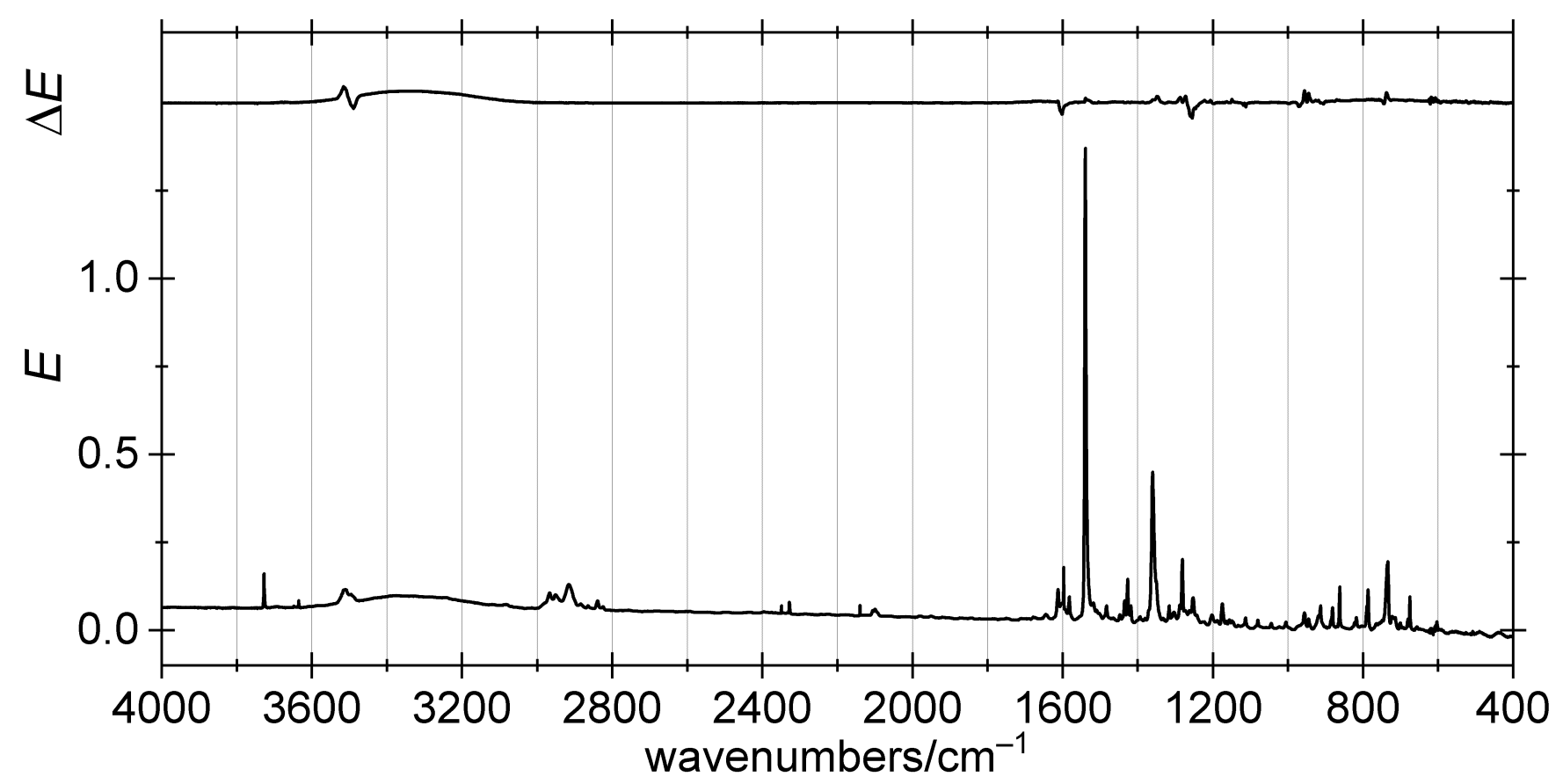

Figure S38: Matrix-isolated $o$-NBA dithiane in $\mathrm{N}_{2}$ at $3 \mathrm{~K}$ after $60 \mathrm{~s}$ irradiation at $313 \mathrm{~nm}$ and $19 \mathrm{~h}$ in the dark; difference trace (before/after $19 \mathrm{~h}$ in the dark) on top.

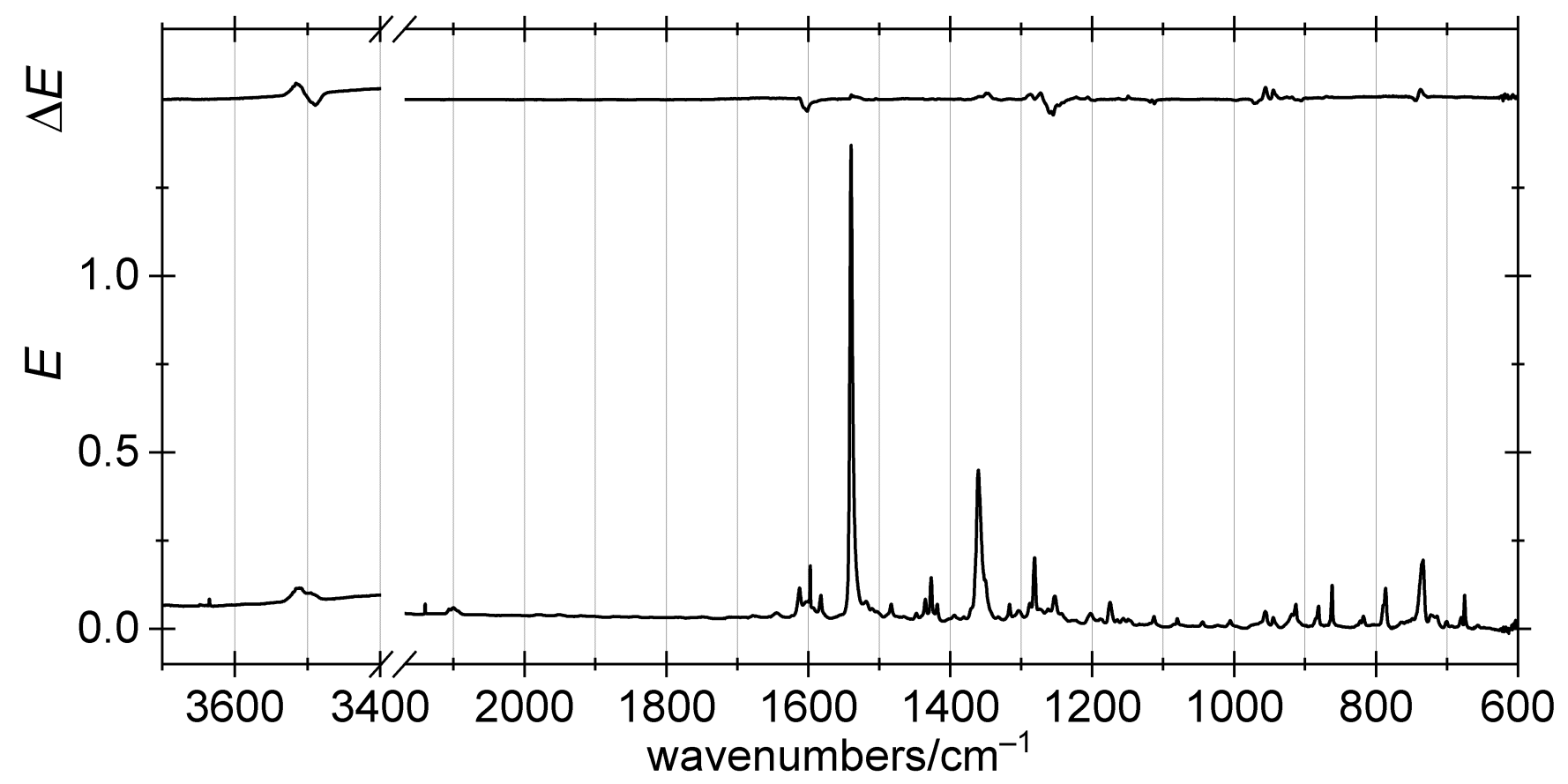

Figure S39: Matrix-isolated $o$-NBA dithiane in $\mathrm{N}_{2}$ at $3 \mathrm{~K}$ after $60 \mathrm{~s}$ irradiation at $313 \mathrm{~nm}$ and $19 \mathrm{~h}$ in the dark (detail); difference trace (before/after $19 \mathrm{~h}$ in the dark) on top. 


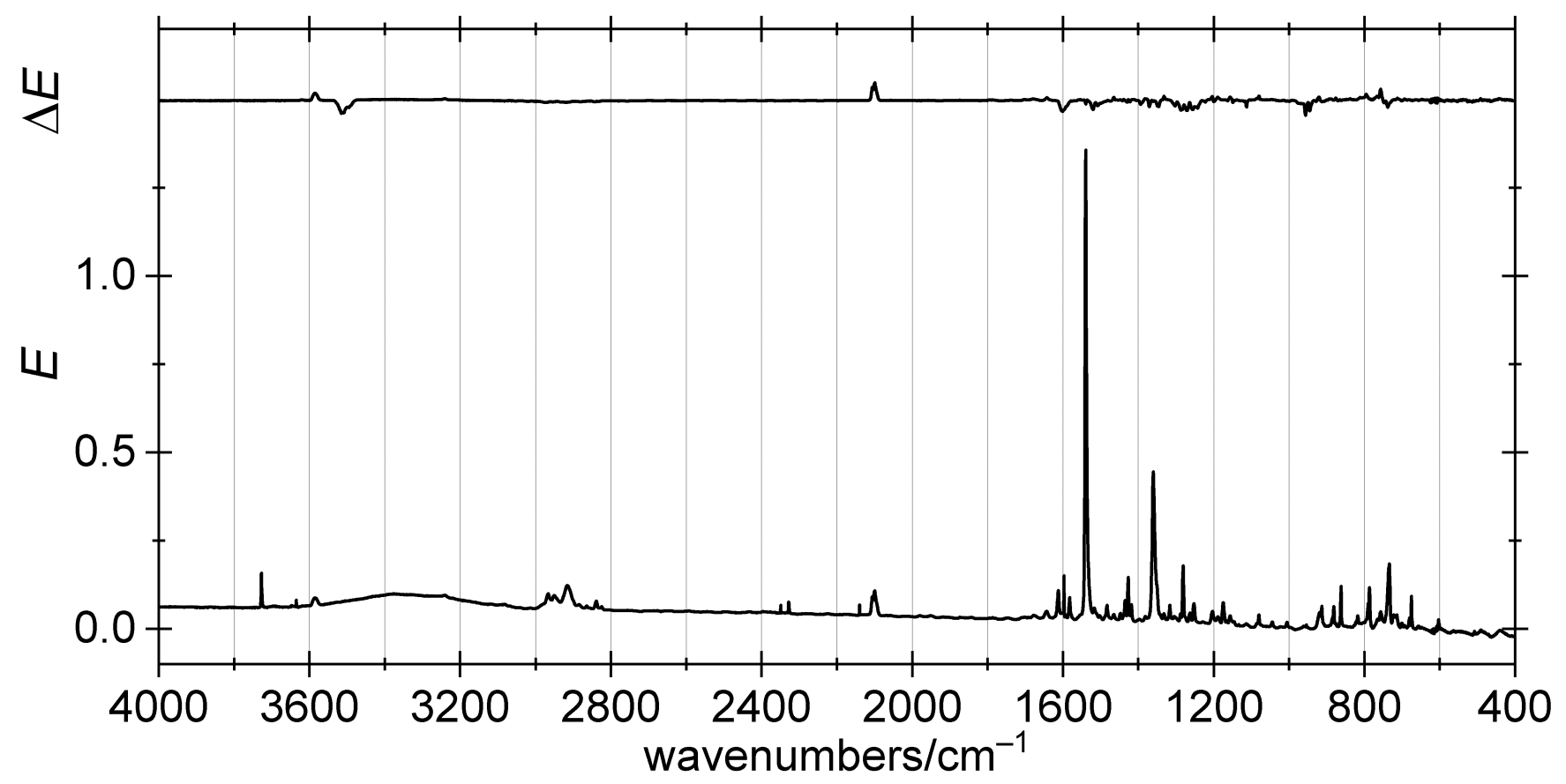

Figure S40: Matrix-isolated $o$-NBA dithiane in $\mathrm{N}_{2}$ at $3 \mathrm{~K}$ after $60 \mathrm{~s}$ irradiation at $313 \mathrm{~nm}, 19 \mathrm{~h}$ in the dark, and $6 \mathrm{~min}$ irradiation at $436 \mathrm{~nm}$; difference trace (before/after irradiation at $436 \mathrm{~nm}$ ) on top.

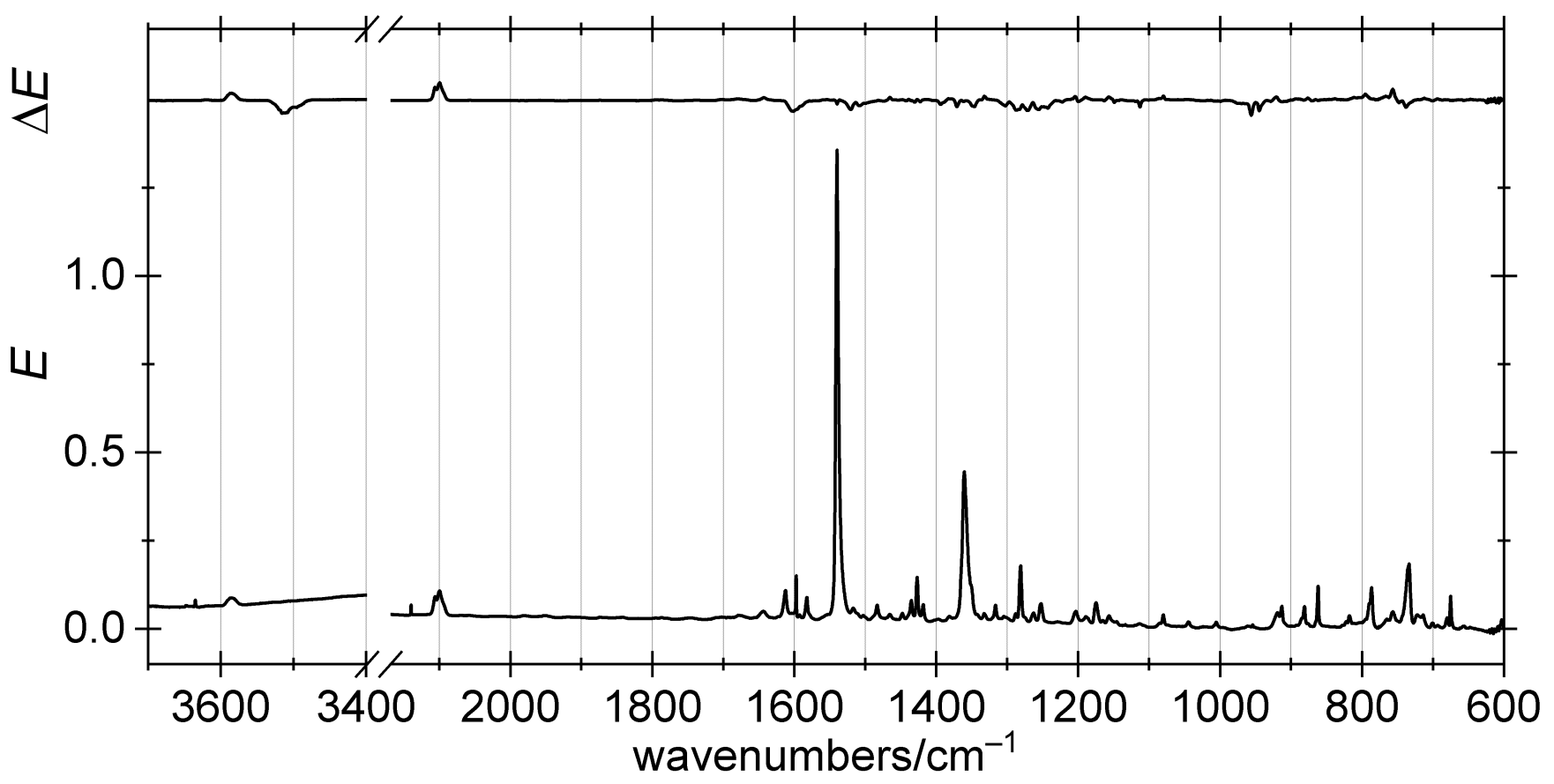

Figure S41: Matrix-isolated $o$-NBA dithiane in $\mathrm{N}_{2}$ at $3 \mathrm{~K}$ after $60 \mathrm{~s}$ irradiation at $313 \mathrm{~nm}, 19 \mathrm{~h}$ in the dark, and $6 \mathrm{~min}$ irradiation at $436 \mathrm{~nm}$ (detail); difference trace (before/after irradiation at $436 \mathrm{~nm}$ ) on top. 


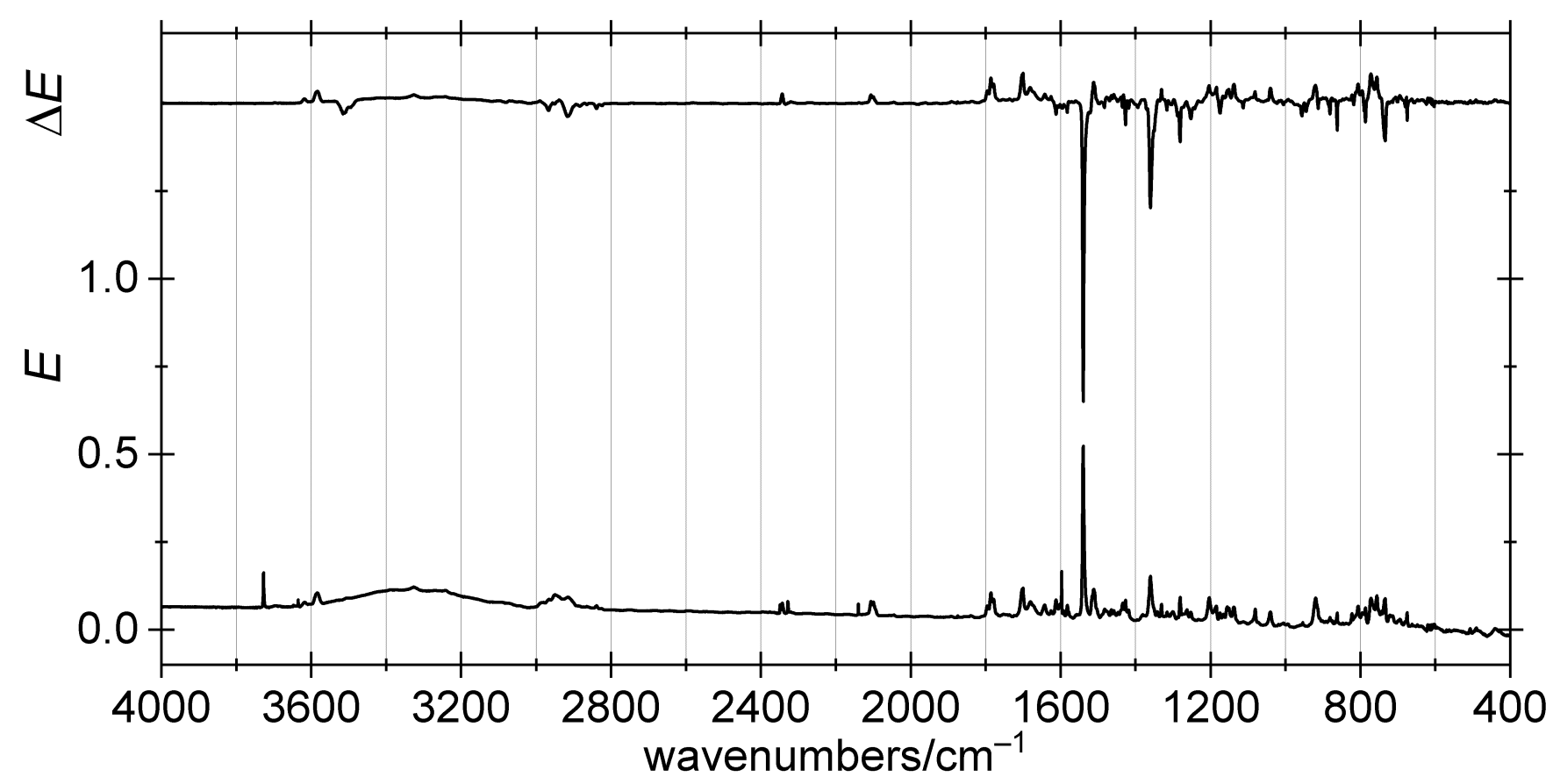

Figure S42: Matrix-isolated $o$-NBA dithiane in $\mathrm{N}_{2}$ at $3 \mathrm{~K}$ after $60 \mathrm{~s}$ irradiation at $313 \mathrm{~nm}, 19 \mathrm{~h}$ in the dark, and $4 \mathrm{~h}$ irradiation at $436 \mathrm{~nm}$; difference trace (before/after irradiation at $436 \mathrm{~nm}$ ) on top.

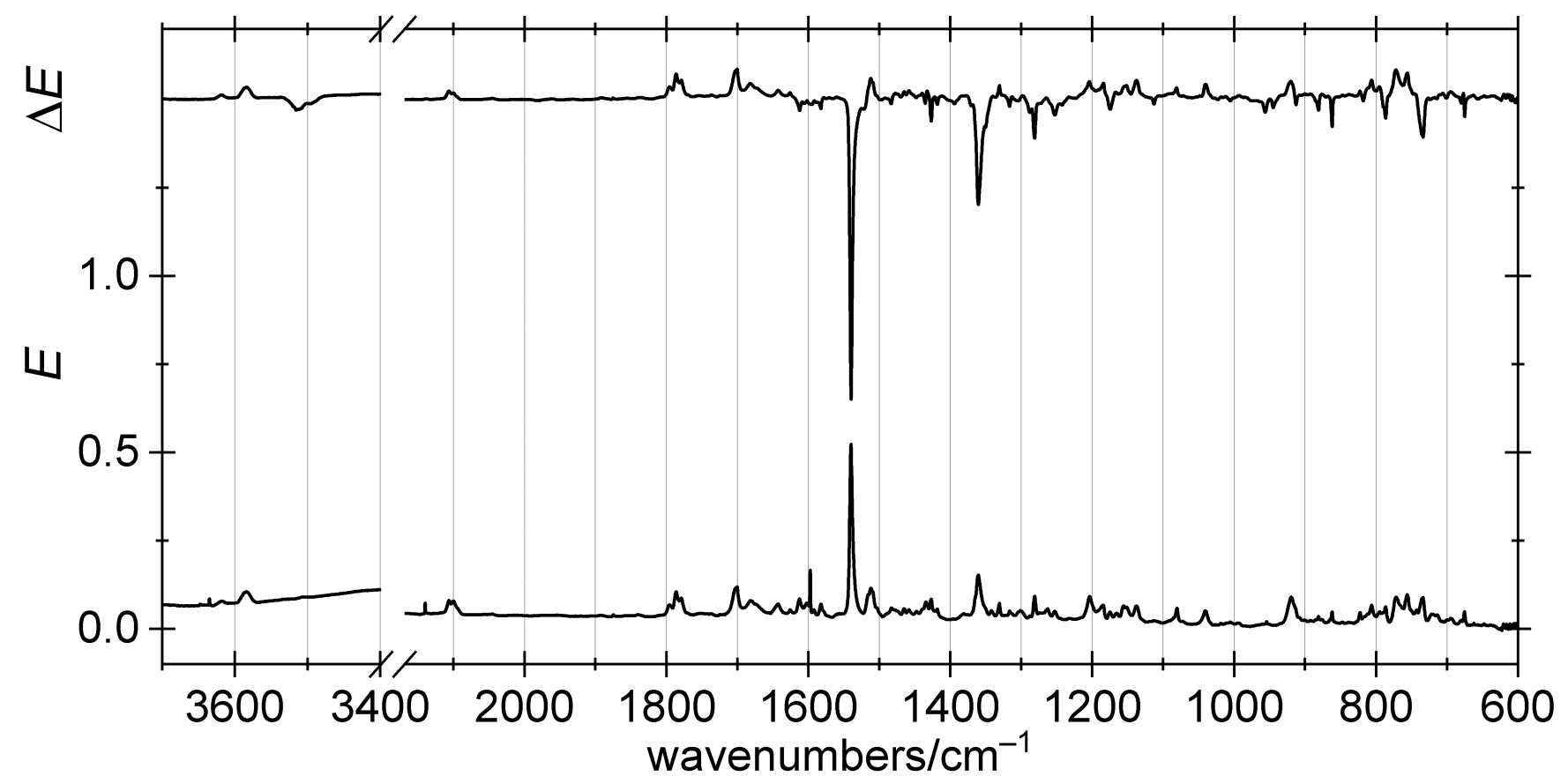

Figure S43: Matrix-isolated $o$-NBA dithiane in $\mathrm{N}_{2}$ at $3 \mathrm{~K}$ after $60 \mathrm{~s}$ irradiation at $313 \mathrm{~nm}, 19 \mathrm{~h}$ in the dark, and $4 \mathrm{~h}$ irradiation at $436 \mathrm{~nm}$ (detail); difference trace (before/after irradiation at $436 \mathrm{~nm}$ ) on top. 
3.2. IR spectra of $d_{1}-o$-NBA dithiane in $\mathrm{N}_{2}$

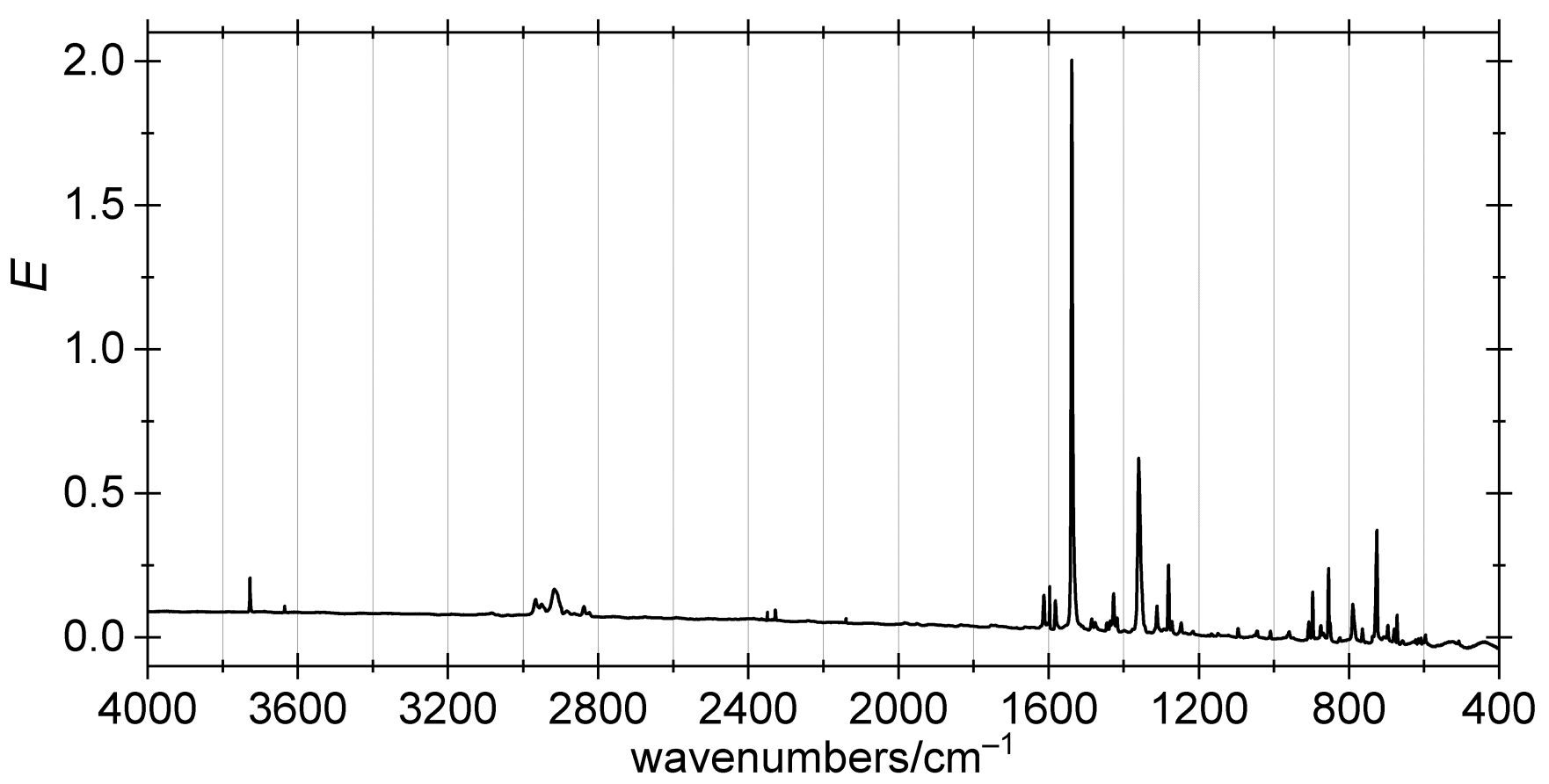

Figure S44: Matrix-isolated $d_{1}-o-\mathrm{NBA}$ dithiane in $\mathrm{N}_{2}$ at $3 \mathrm{~K}$.

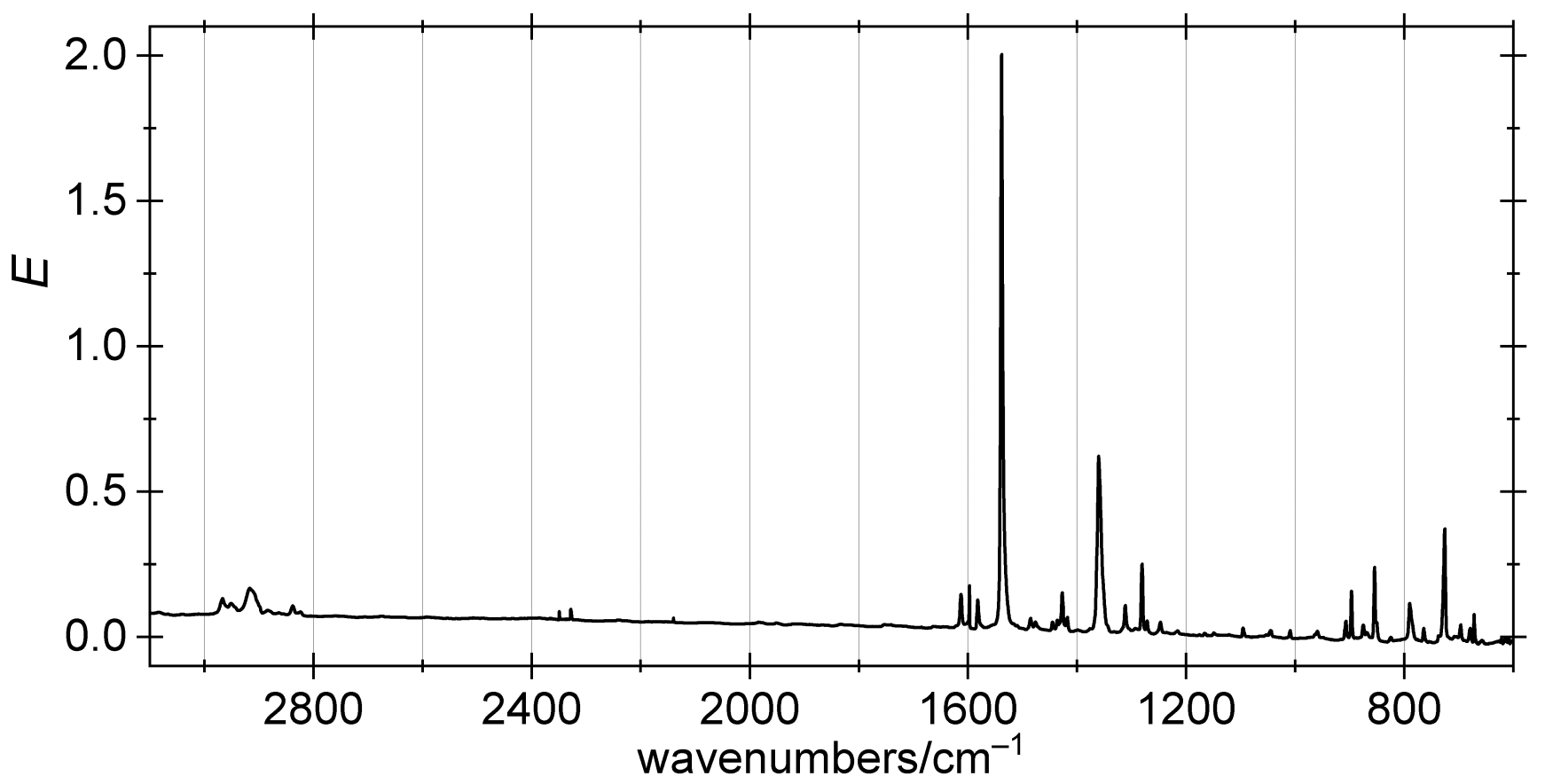

Figure S45: Matrix-isolated $d_{1}-o-\mathrm{NBA}$ dithiane in $\mathrm{N}_{2}$ at $3 \mathrm{~K}$ (detail). 


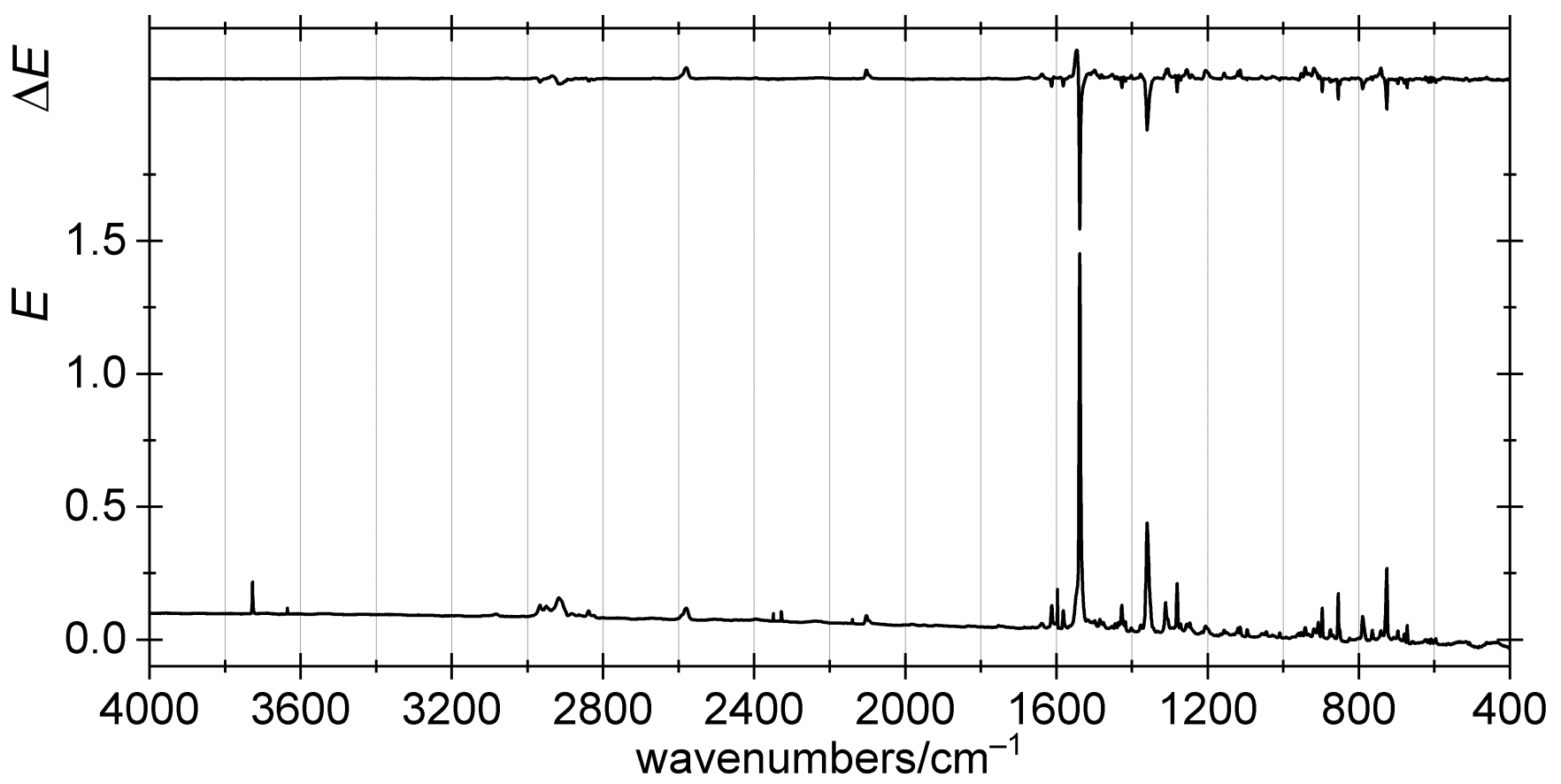

Figure S46: Matrix-isolated $d_{1}-o$-NBA dithiane in $\mathrm{N}_{2}$ at $3 \mathrm{~K}$ after $60 \mathrm{~s}$ irradiation at $313 \mathrm{~nm}$; difference trace (before/after irradiation at $313 \mathrm{~nm}$ ) on top.

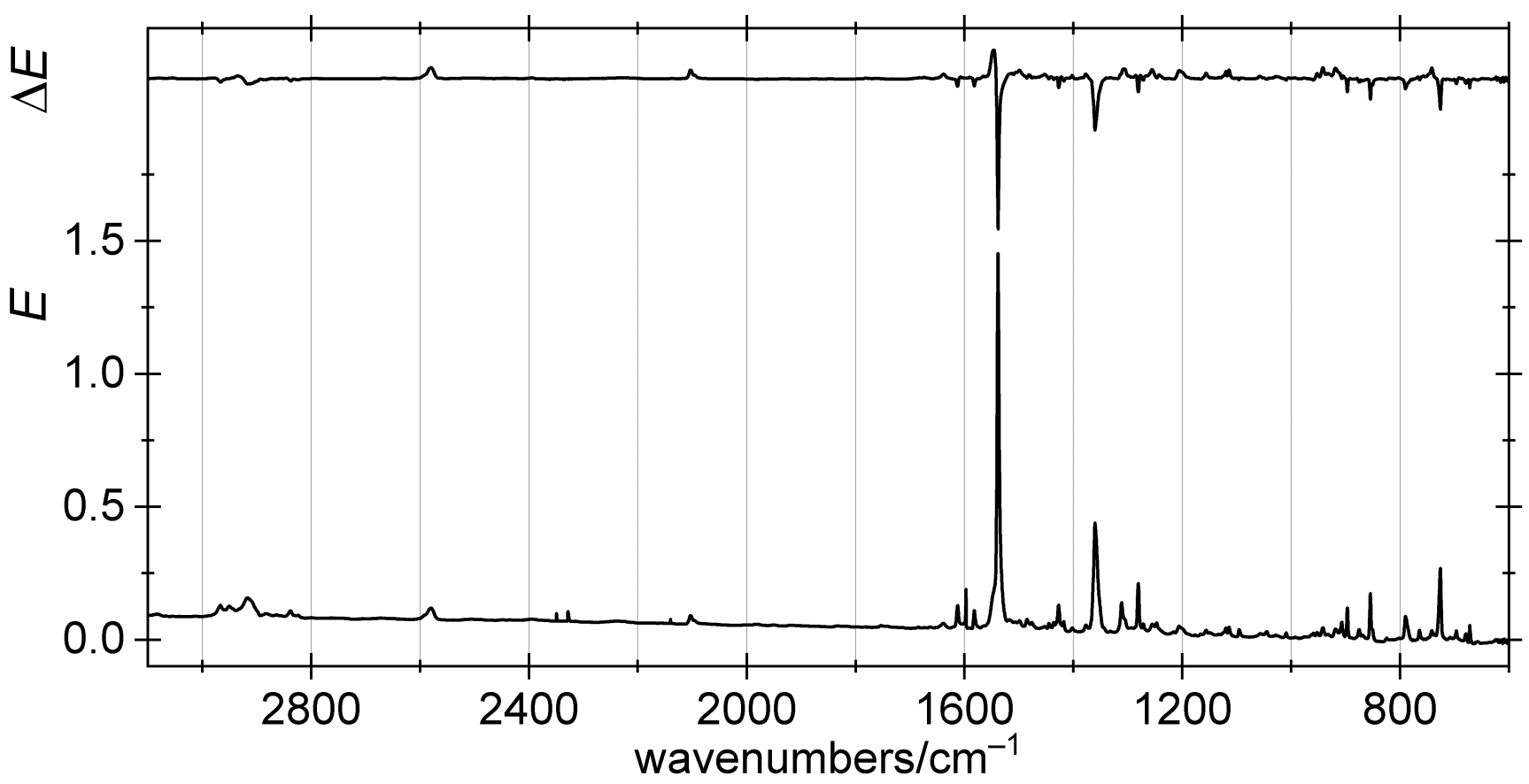

Figure S47: Matrix-isolated $d_{1}-o$-NBA dithiane in $\mathrm{N}_{2}$ at $3 \mathrm{~K}$ after $60 \mathrm{~s}$ irradiation at $313 \mathrm{~nm}$ (detail); difference trace (before/after irradiation at $313 \mathrm{~nm}$ ) on top. 


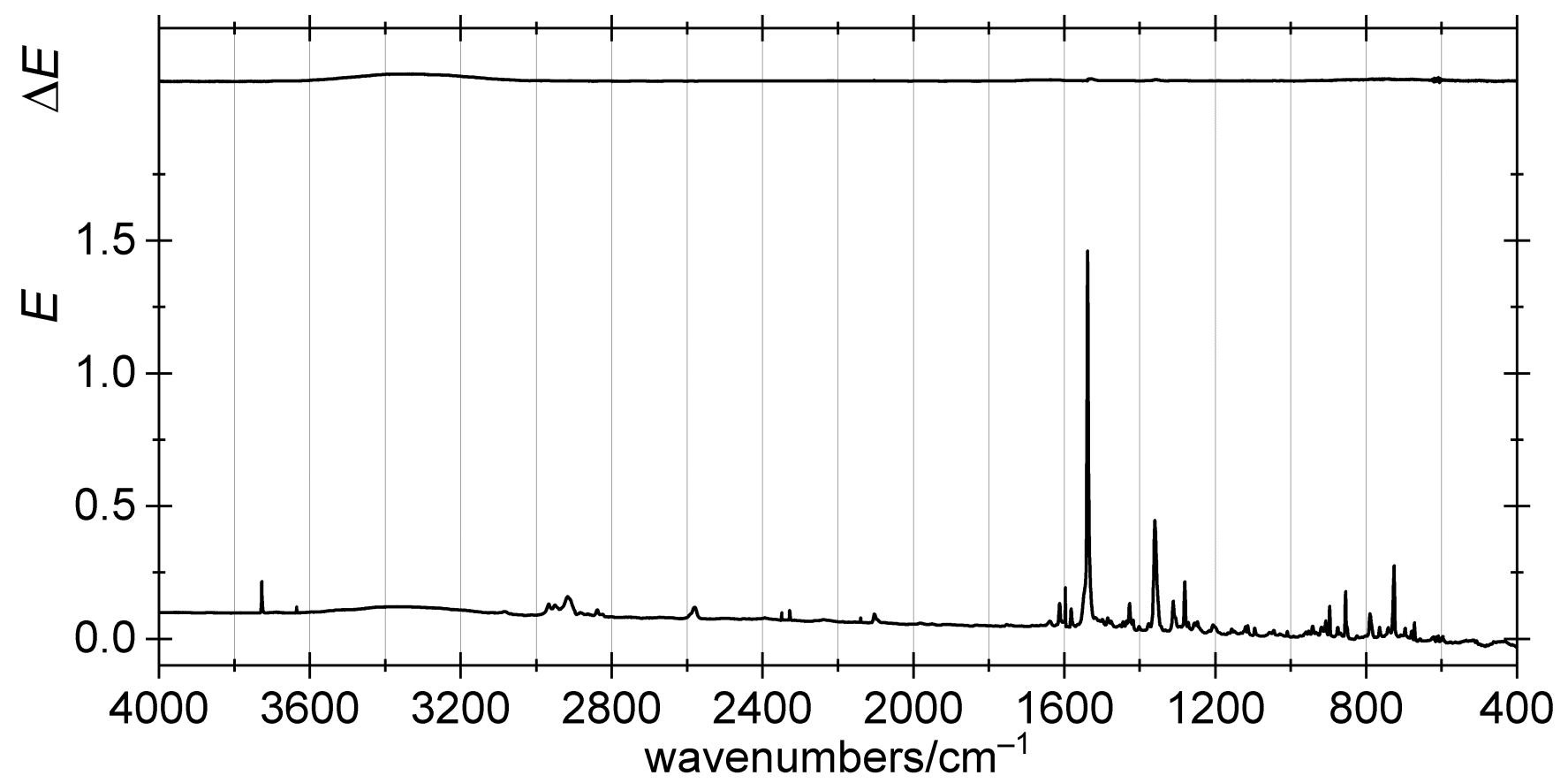

Figure S48: Matrix-isolated $d_{1}-o$-NBA dithiane in $\mathrm{N}_{2}$ at $3 \mathrm{~K}$ after $60 \mathrm{~s}$ irradiation at $313 \mathrm{~nm}$ and $16 \mathrm{~h}$ in the dark; difference trace (before/after $16 \mathrm{~h}$ in the dark) on top.

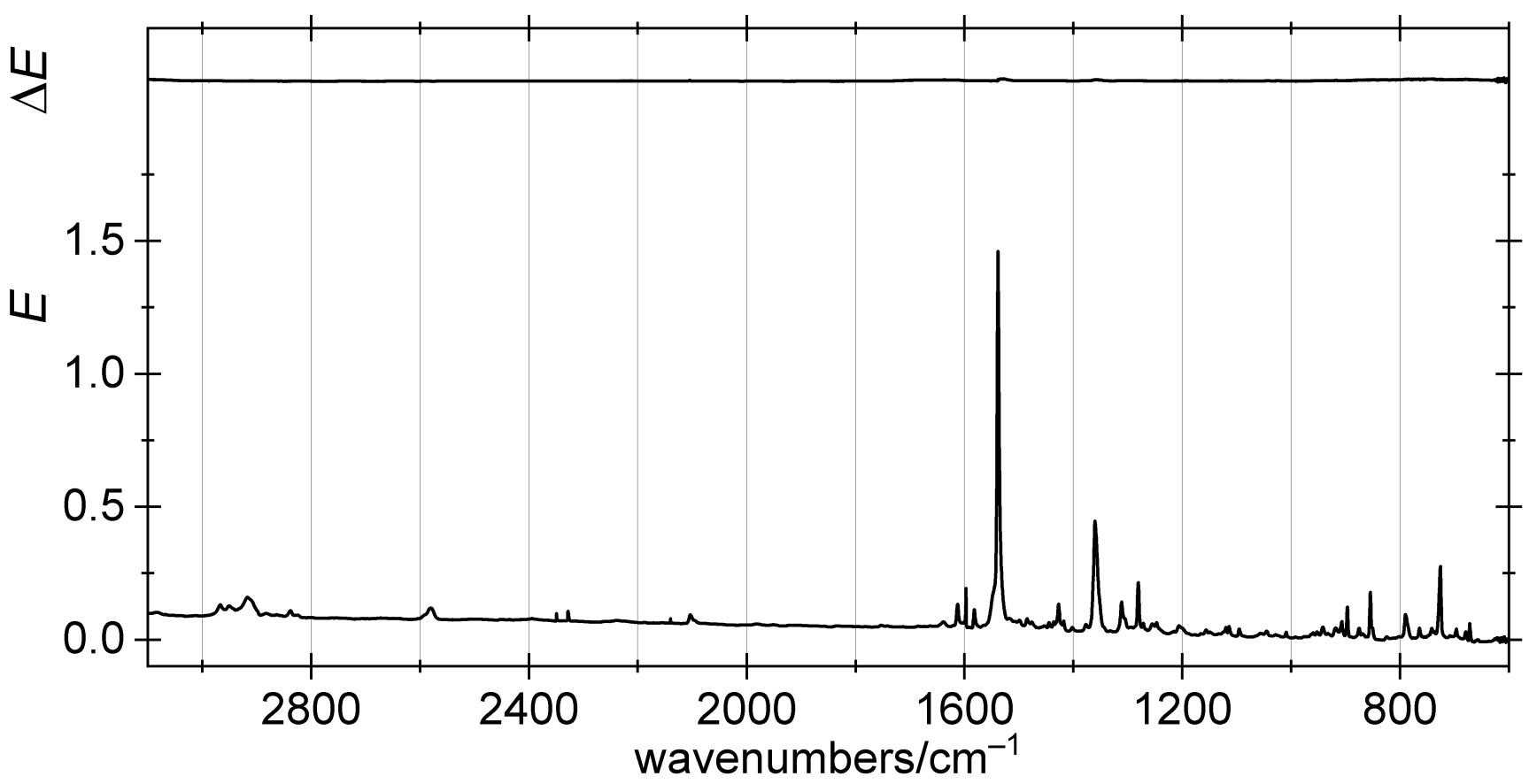

Figure S49: Matrix-isolated $d_{1}-o$-NBA dithiane in $\mathrm{N}_{2}$ at $3 \mathrm{~K}$ after $60 \mathrm{~s}$ irradiation at $313 \mathrm{~nm}$ and $16 \mathrm{~h}$ in the dark (detail); difference trace (before/after $16 \mathrm{~h}$ in the dark) on top. 


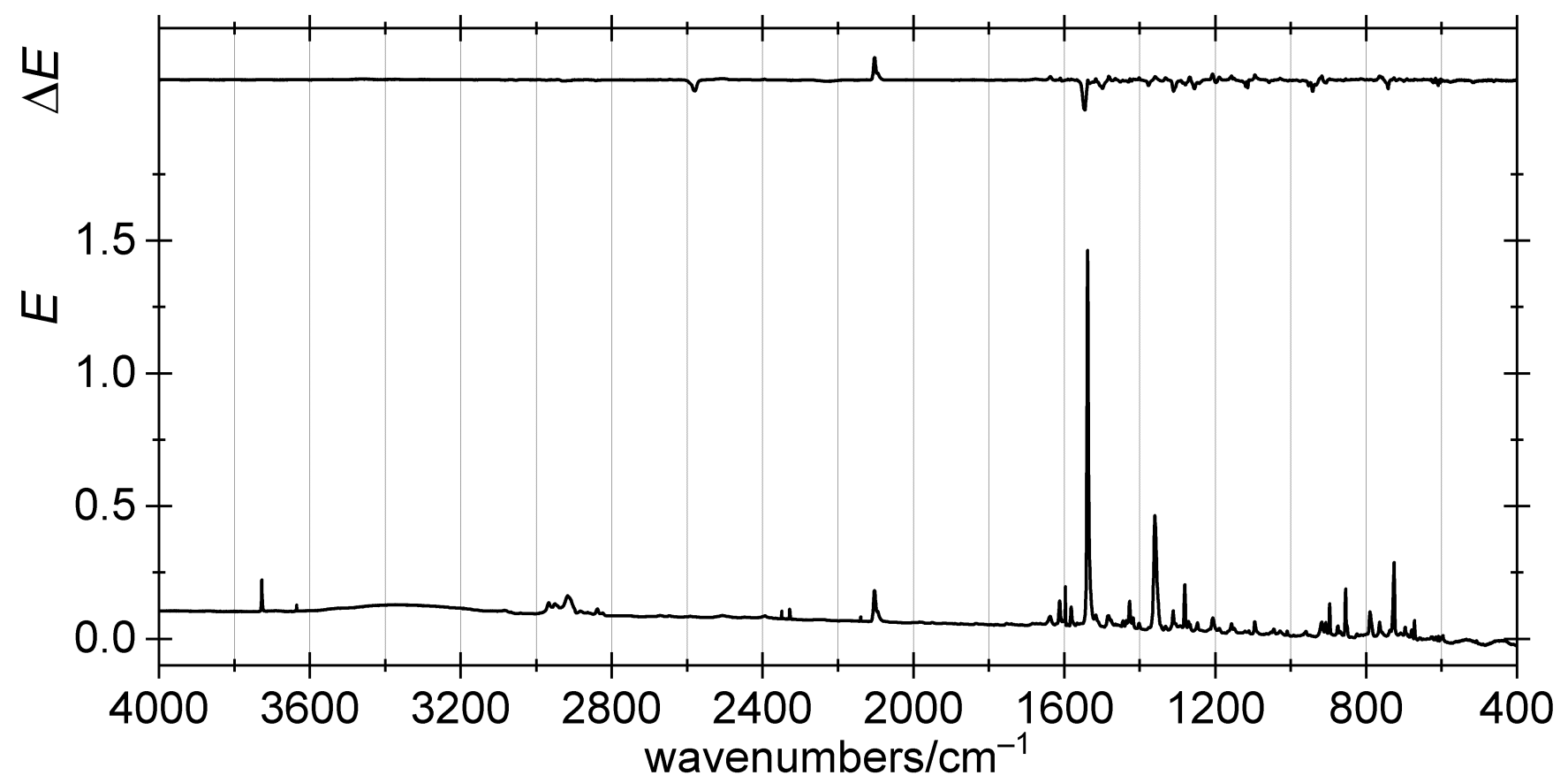

Figure S50: Matrix-isolated $d_{1}-o$-NBA dithiane in $\mathrm{N}_{2}$ at $3 \mathrm{~K}$ after $60 \mathrm{~s}$ irradiation at $313 \mathrm{~nm}, 16 \mathrm{~h}$ in the dark, and 4 min irradiation at $436 \mathrm{~nm}$; difference trace (before/after irradiation at $436 \mathrm{~nm}$ ) on top.

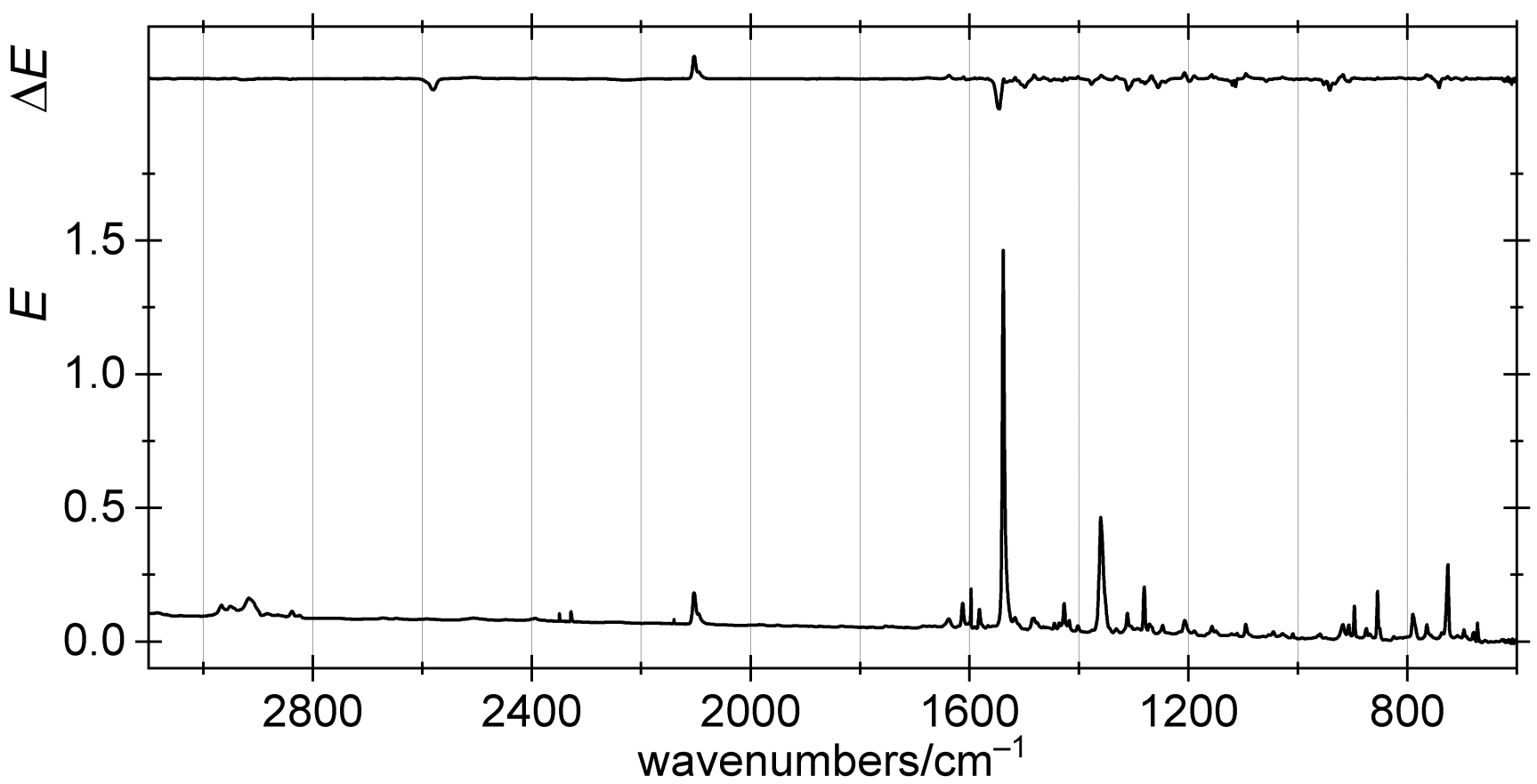

Figure S51: Matrix-isolated $d_{1}-o$-NBA dithiane in $\mathrm{N}_{2}$ at $3 \mathrm{~K}$ after $60 \mathrm{~s}$ irradiation at $313 \mathrm{~nm}, 16 \mathrm{~h}$ in the dark, and $4 \mathrm{~min}$ irradiation at $436 \mathrm{~nm}$ (detail); difference trace (before/after irradiation at $436 \mathrm{~nm}$ ) on top. 


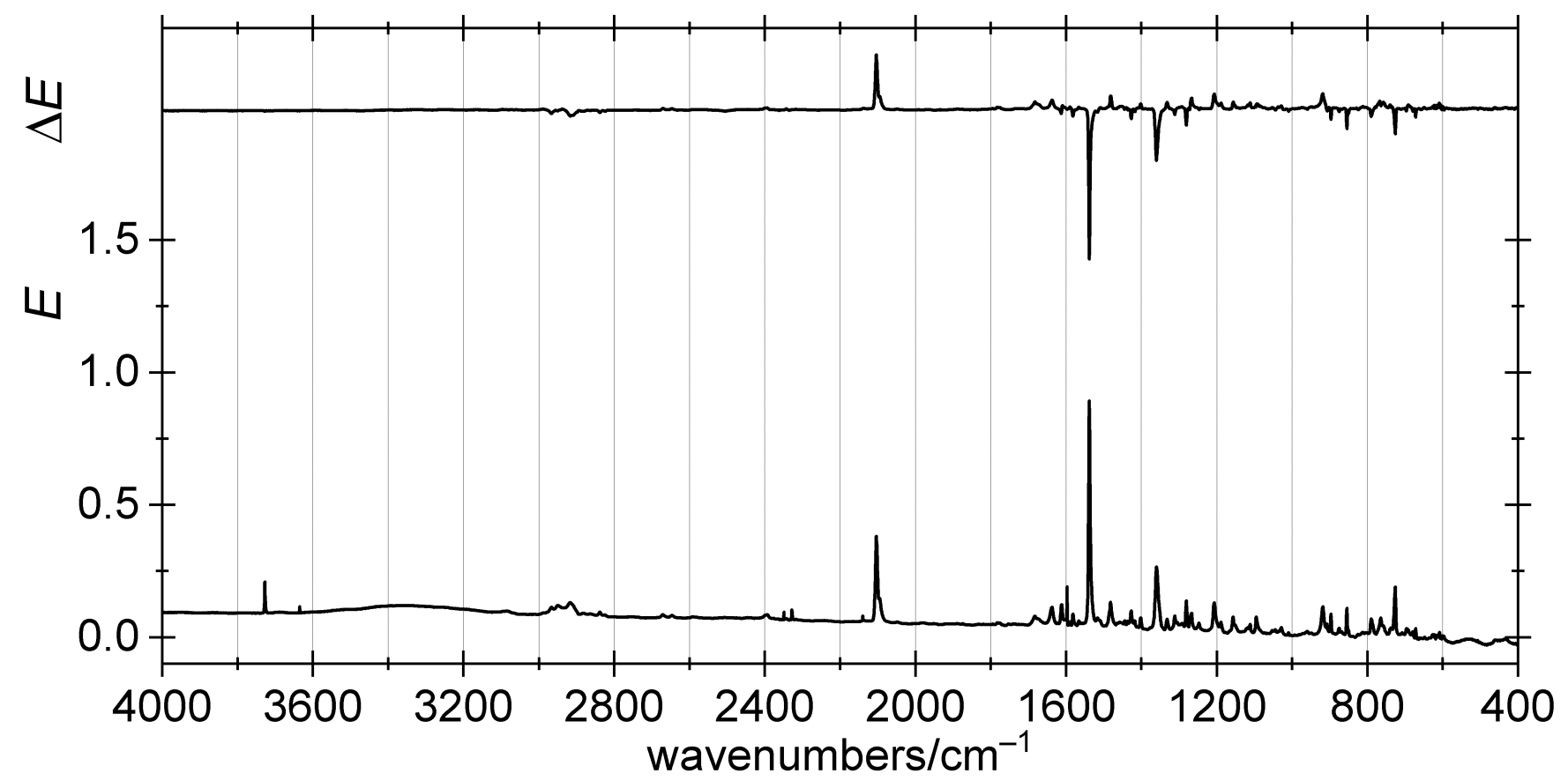

Figure S52: Matrix-isolated $d_{1}-o$-NBA dithiane in $\mathrm{N}_{2}$ at $3 \mathrm{~K}$ after $60 \mathrm{~s}$ irradiation at $313 \mathrm{~nm}, 16 \mathrm{~h}$ in the dark, 4 min irradiation at $436 \mathrm{~nm}$, and $20 \mathrm{~min}$ irradiation at $405 \mathrm{~nm}$; difference trace (before/after irradiation at $405 \mathrm{~nm}$ ) on top.

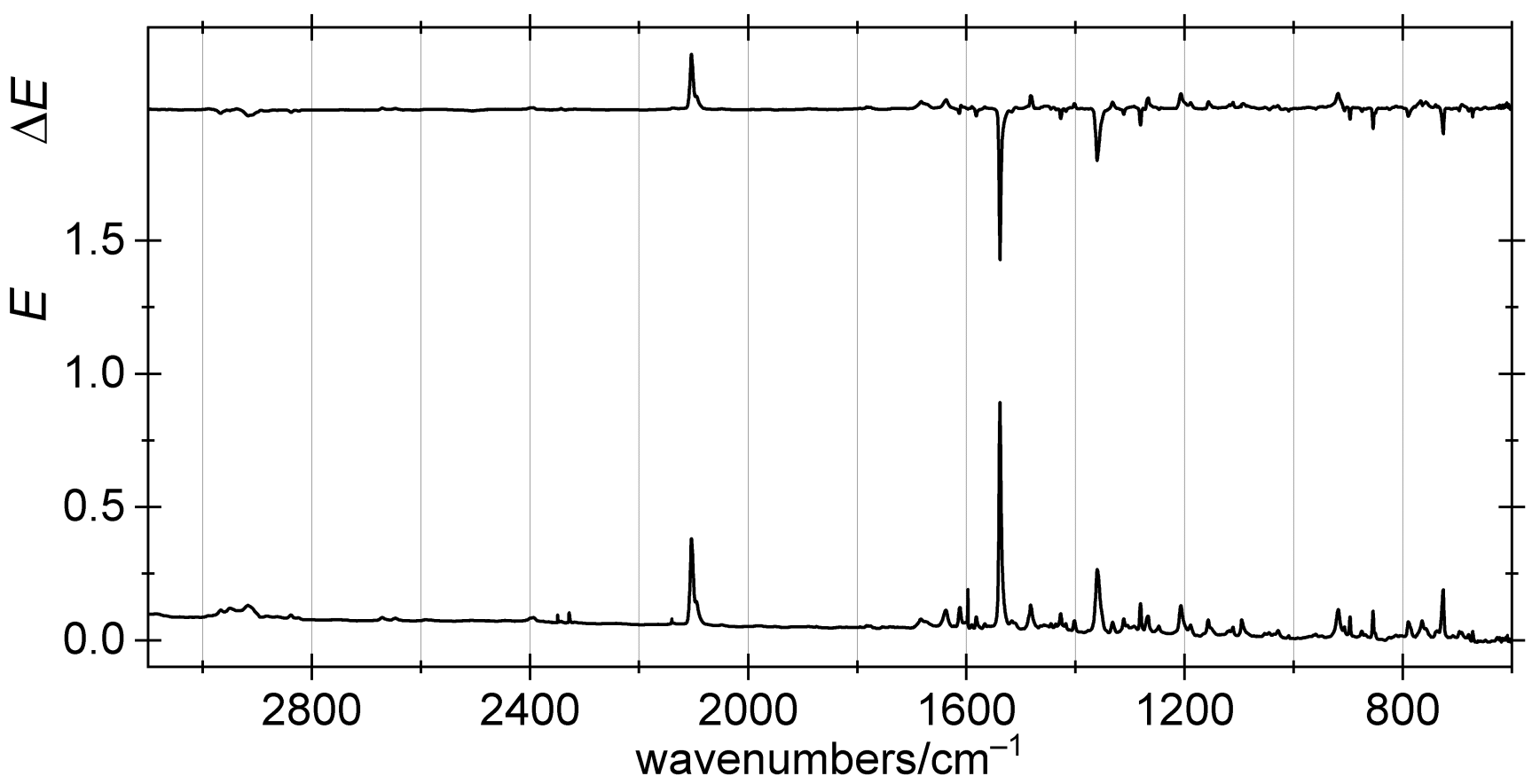

Figure S53: Matrix-isolated $d_{1}-o$-NBA dithiane in $\mathrm{N}_{2}$ at $3 \mathrm{~K}$ after $60 \mathrm{~s}$ irradiation at $313 \mathrm{~nm}, 16 \mathrm{~h}$ in the dark, $4 \mathrm{~min}$ irradiation at $436 \mathrm{~nm}$, and $20 \mathrm{~min}$ irradiation at $405 \mathrm{~nm}$ (detail); difference trace (before/after irradiation at $405 \mathrm{~nm}$ ) on top. 


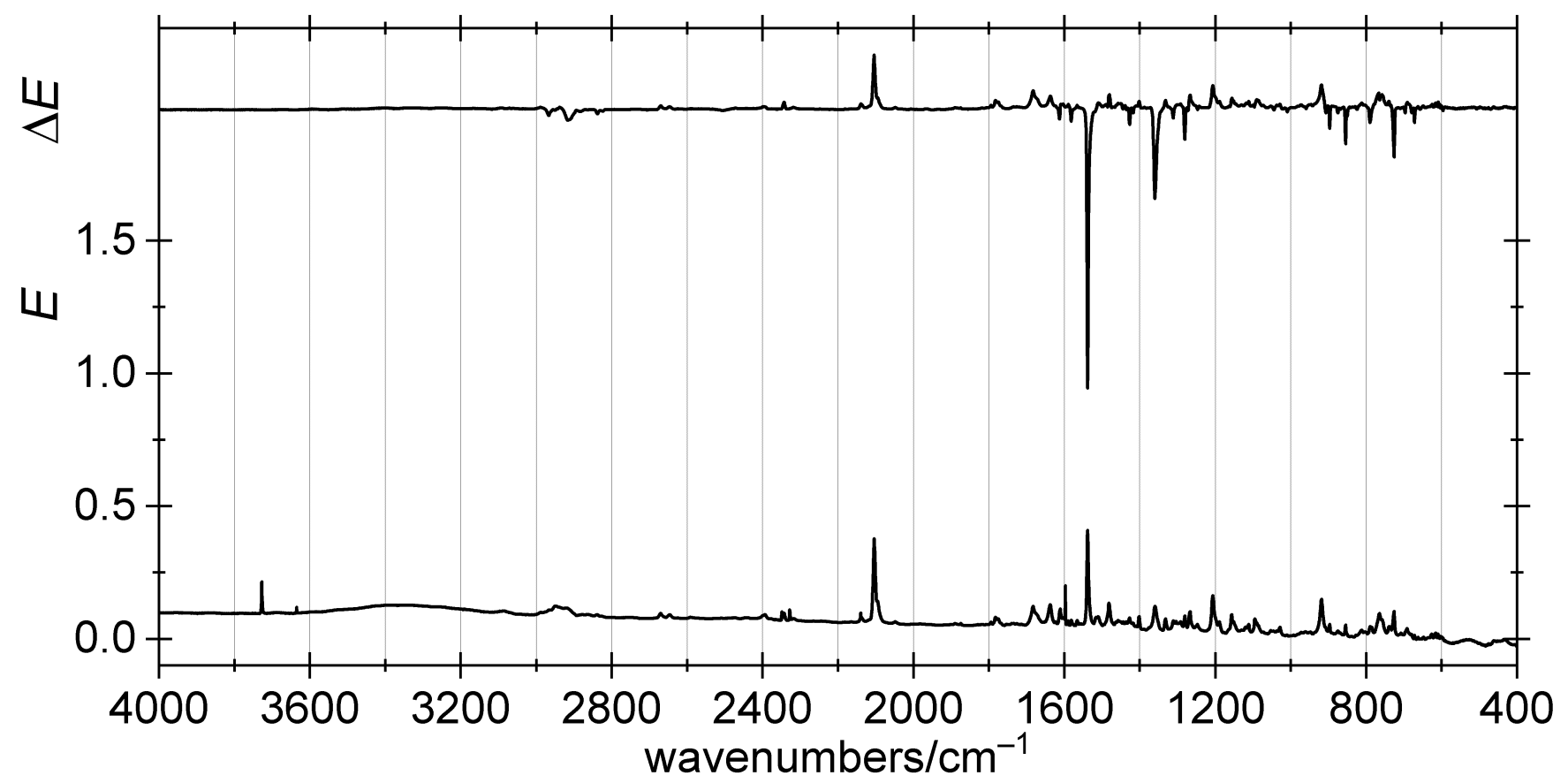

Figure S54: Matrix-isolated $d_{1}-o$-NBA dithiane in $\mathrm{N}_{2}$ at $3 \mathrm{~K}$ after $60 \mathrm{~s}$ irradiation at $313 \mathrm{~nm}, 16 \mathrm{~h}$ in the dark, 4 min irradiation at $436 \mathrm{~nm}$, and $60 \mathrm{~min}$ irradiation at $405 \mathrm{~nm}$; difference trace (before/after irradiation at $405 \mathrm{~nm}$ ) on top.

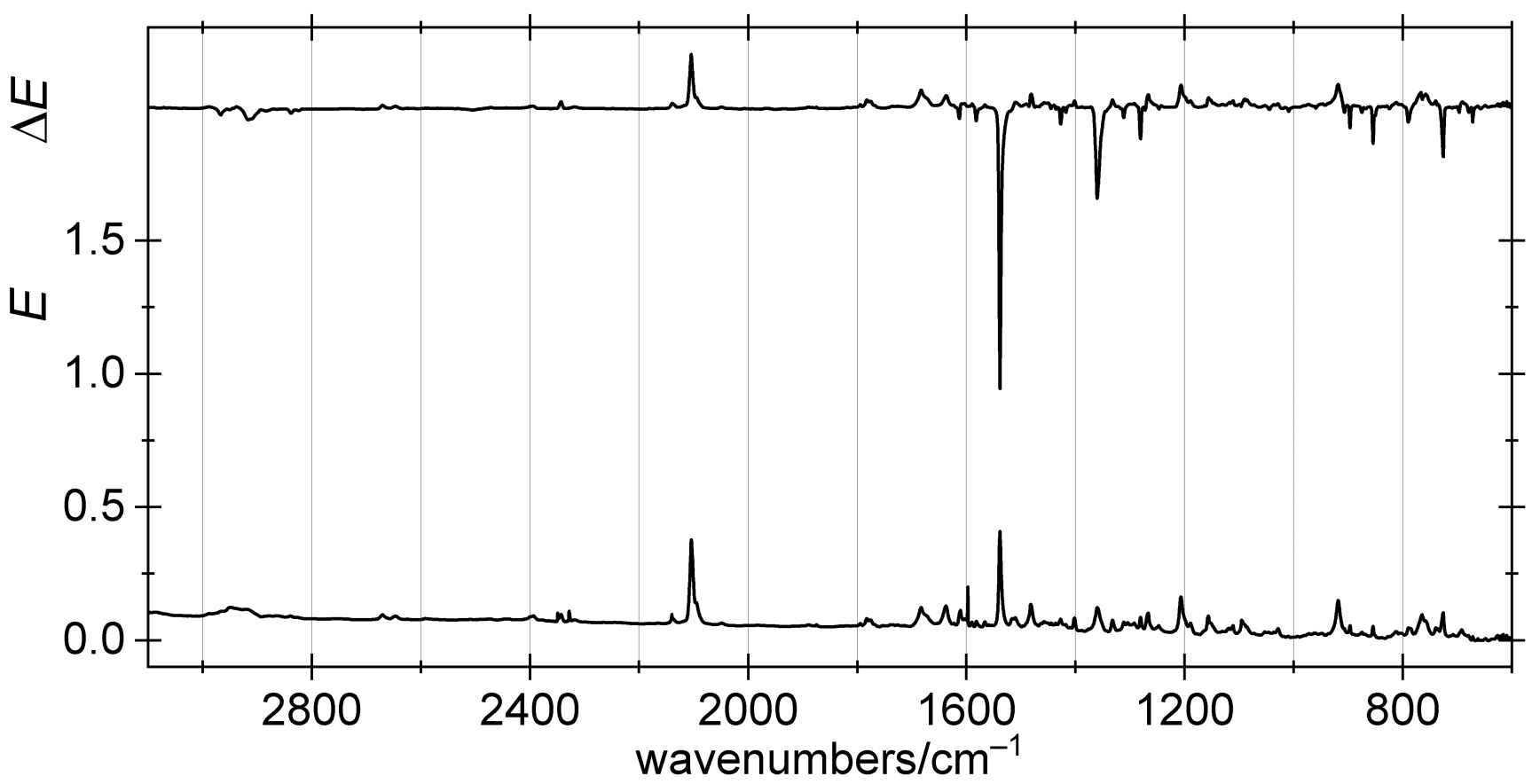

Figure S55: Matrix-isolated $d_{1}-o$-NBA dithiane in $\mathrm{N}_{2}$ at $3 \mathrm{~K}$ after $60 \mathrm{~s}$ irradiation at $313 \mathrm{~nm}, 16 \mathrm{~h}$ in the dark, $4 \mathrm{~min}$ irradiation at $436 \mathrm{~nm}$, and $60 \mathrm{~min}$ irradiation at $405 \mathrm{~nm}$ (detail); difference trace (before/after irradiation at $405 \mathrm{~nm}$ ) on top. 


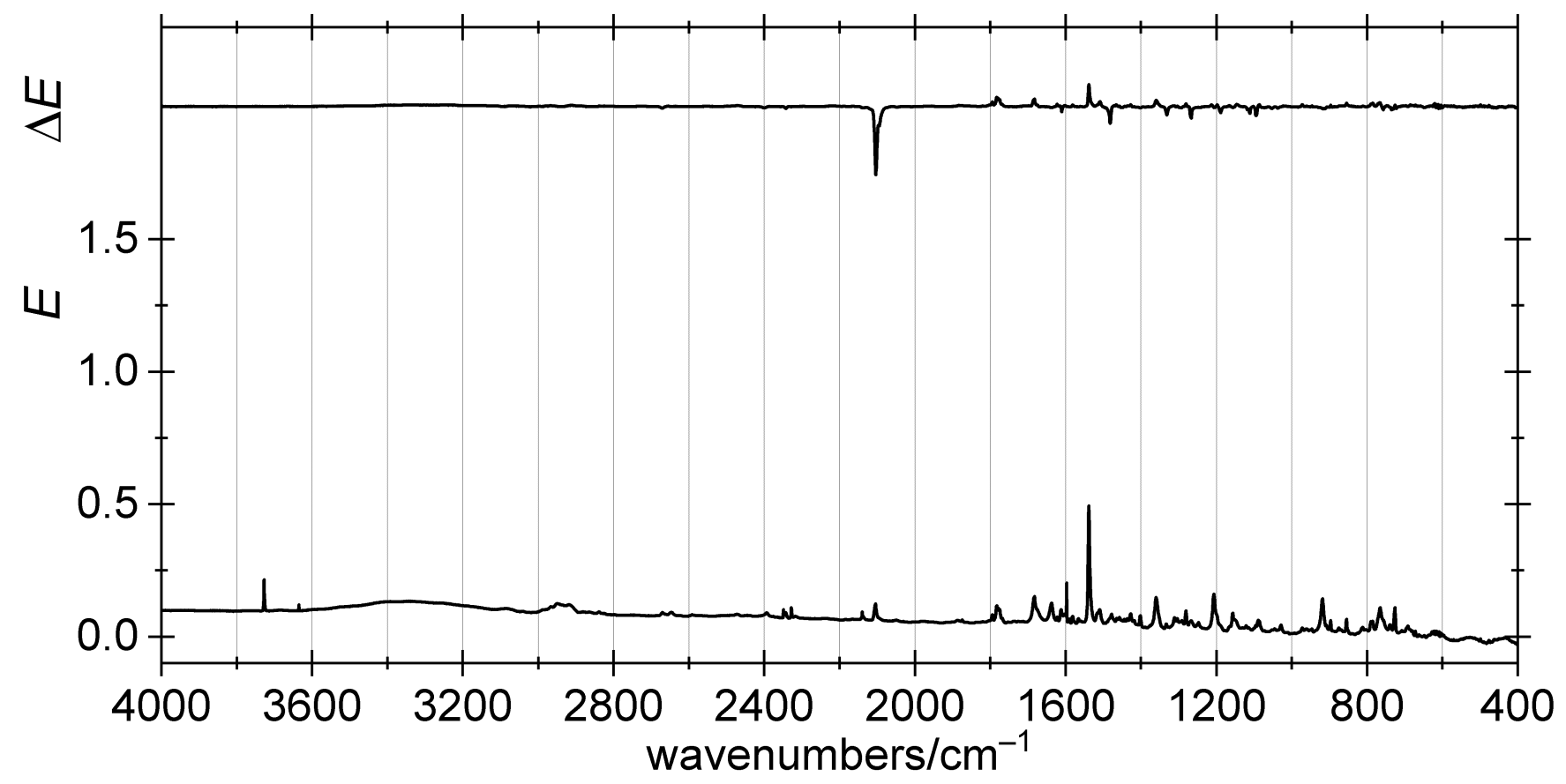

Figure S56: Matrix-isolated $d_{1}-o-\mathrm{NBA}$ dithiane in $\mathrm{N}_{2}$ at $3 \mathrm{~K}$ after $60 \mathrm{~s}$ irradiation at $313 \mathrm{~nm}, 16 \mathrm{~h}$ in the dark, $4 \mathrm{~min}$ irradiation at $436 \mathrm{~nm}, 60 \mathrm{~min}$ irradiation at $405 \mathrm{~nm}$, and $125 \mathrm{~min}$ irradiation at $546 \mathrm{~nm}$; difference trace (before/after irradiation at $546 \mathrm{~nm}$ ) on top.

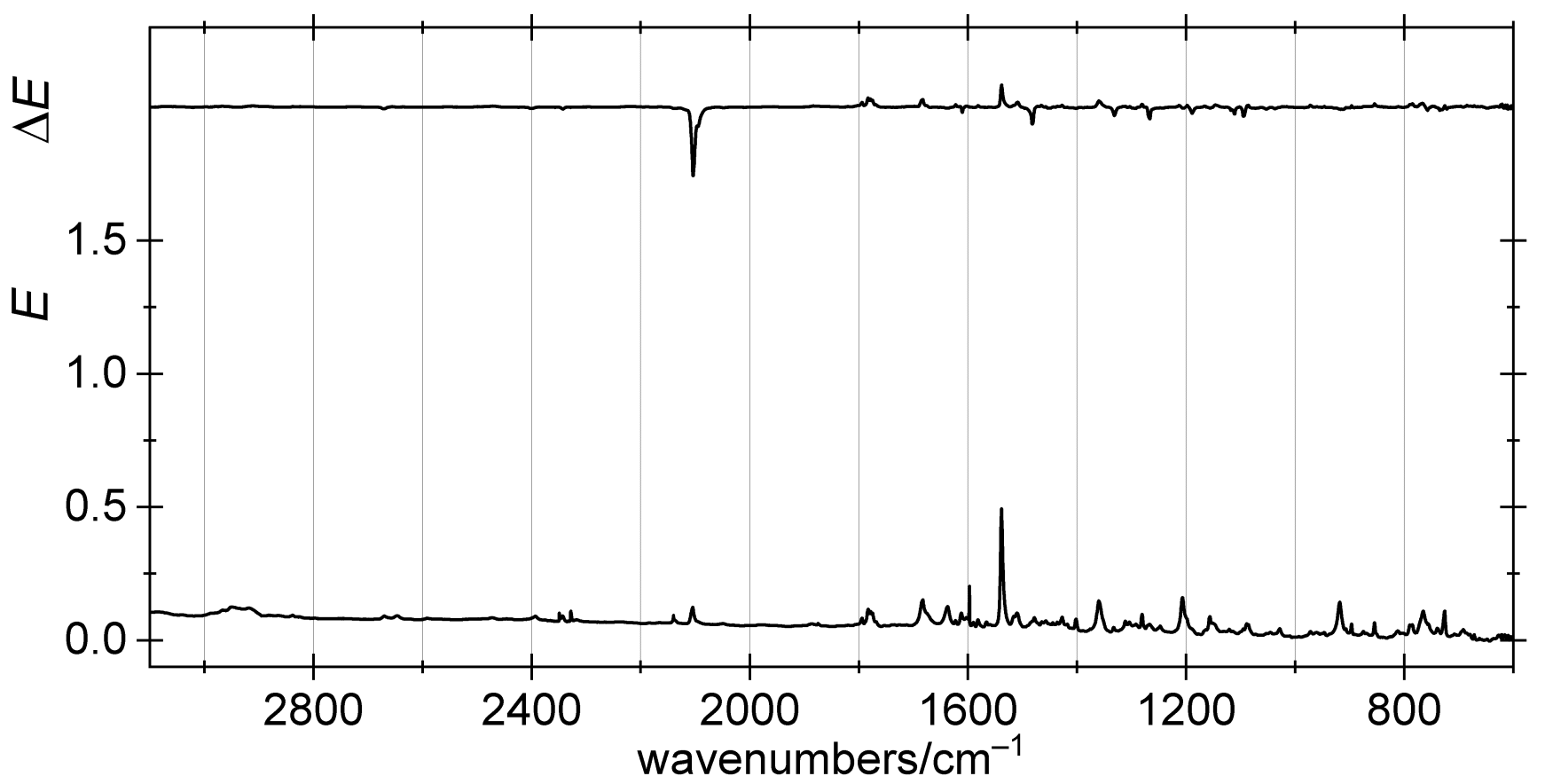

Figure S57: Matrix-isolated $d_{1}-o$-NBA dithiane in $\mathrm{N}_{2}$ at $3 \mathrm{~K}$ after $60 \mathrm{~s}$ irradiation at $313 \mathrm{~nm}, 16 \mathrm{~h}$ in the dark, 4 min irradiation at $436 \mathrm{~nm}, 60 \mathrm{~min}$ irradiation at $405 \mathrm{~nm}$, and $125 \mathrm{~min}$ irradiation at $546 \mathrm{~nm}$ (detail); difference trace (before/after irradiation at $546 \mathrm{~nm}$ ) on top. 
3.3. UV/Vis spectra of $o$-NBA dithiane in $\mathrm{N}_{2}$

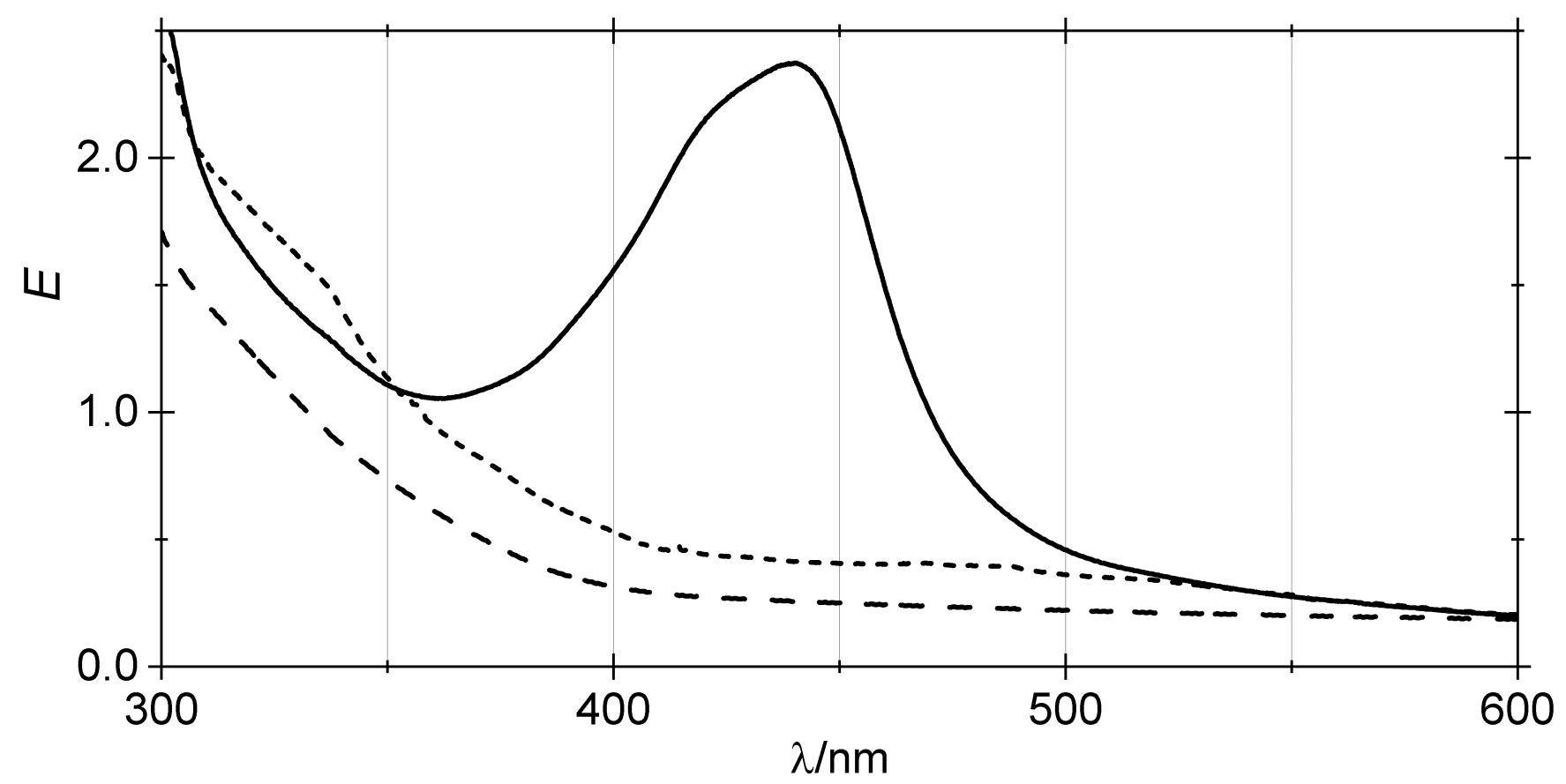

Figure S58: Matrix-isolated $o$-NBA dithiane in $\mathrm{N}_{2}$ at $3 \mathrm{~K}$ after deposition (dashed trace), after $60 \mathrm{~s}$ irradiation at $313 \mathrm{~nm}$ (solid trace), and $6 \mathrm{~min}$ irradiation at $436 \mathrm{~nm}$ (short-dashed trace).

3.4. UV/Vis spectra of $d_{1}-o$-NBA dithiane in $\mathrm{N}_{2}$

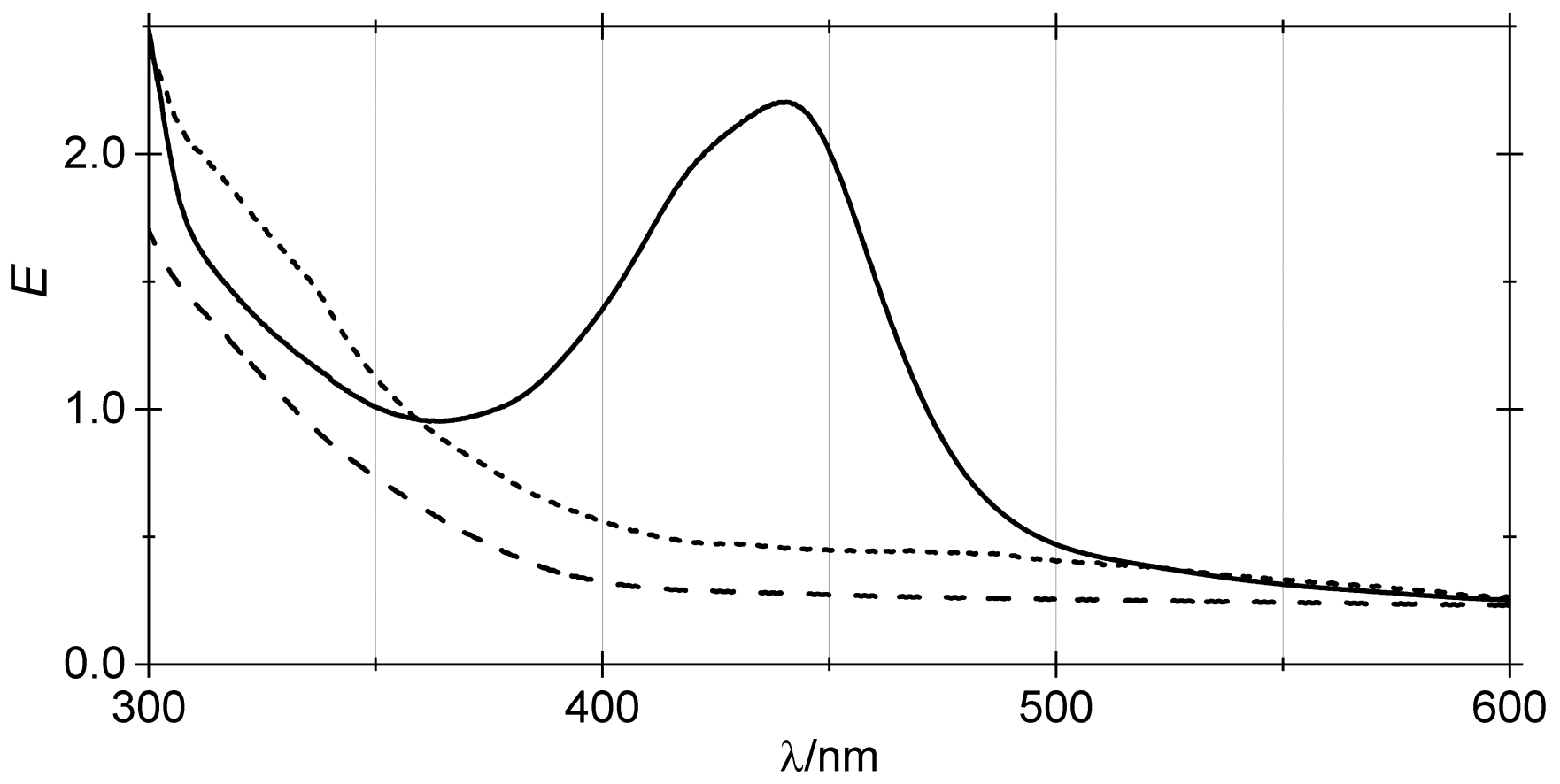

Figure S59: Matrix-isolated $d_{1}-o$-NBA dithiane in $\mathrm{N}_{2}$ at $3 \mathrm{~K}$ after deposition (dashed trace), after $60 \mathrm{~s}$ irradiation at $313 \mathrm{~nm}$ (solid trace), and $4 \mathrm{~min}$ irradiation at $436 \mathrm{~nm}$ (short-dashed trace). 
All starting materials were purchased from commercial suppliers and used for synthesis without further purification. Solvents for column chromatography, extractions, and filtrations were distilled prior to use. All NMR spectra were recorded on Bruker AV 400 or AV 400HD spectrometers. Chemical shifts $(\delta)$ are given in parts per million ( $\mathrm{ppm})$ relative to TMS $(\delta=0.00 \mathrm{ppm})$ as internal standard or to the respective solvent residual peak of $\mathrm{D}_{2} \mathrm{O}(\delta=4.79 \mathrm{ppm}) .{ }^{13} \mathrm{C}\left\{{ }^{1} \mathrm{H}\right\}$ NMR spectra in $\mathrm{D}_{2} \mathrm{O}$ are calibrated to 3-(trimethylsilyl)propionic-2,2,3,3-d $d_{4}$ acid sodium salt (TSPA).

\section{1. (Bromodimethyl)sulfonium bromide}

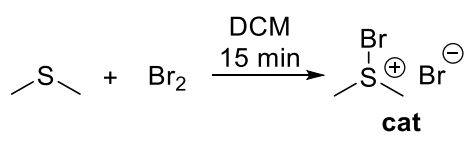

$10 \mathrm{~mL}$ (200 mmol) bromine were diluted in $40 \mathrm{~mL}$ dry dichloromethane (DCM). A mixture of $15 \mathrm{~mL}$ (200 mmol) dimethyl sulfide in $40 \mathrm{~mL}$ DCM was added dropwise over $15 \mathrm{~min}$ at $\mathrm{r}$. $\mathrm{t}$. The resulting orange powder was washed with hexane and dried in vacuo over $\mathrm{P}_{4} \mathrm{O}_{10}(0.18 \mathrm{~mol}, 40.3 \mathrm{~g}, 92 \%)$. ${ }^{1} \mathrm{H}$ NMR (400 MHz, $\left.\mathrm{D}_{2} \mathrm{O}\right): \delta=3.56(\mathrm{~s}, 6 \mathrm{H}) \mathrm{ppm} .{ }^{13} \mathrm{C} \mathrm{NMR}\left(100 \mathrm{MHz}, \mathrm{D}_{2} \mathrm{O}\right): \delta=38.7 \mathrm{ppm}$.

\subsection{2-(2-Nitrophenyl)-1,3-dithiane}

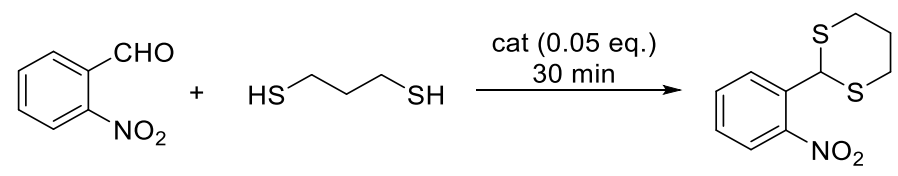

$1.81 \mathrm{~g}$ (12 mmol) 2-nitrobenzaldehyde were dissolved in $1.3 \mathrm{~mL}$ (13.2 mmol) 1,3-propane dithiol. $138.9 \mathrm{mg}(0.60 \mathrm{mmol}, 0.05$ eq.) cat were added to the yellow-green solution. After $30 \mathrm{~min}$, a yellow gel formed, which was heated to $100{ }^{\circ} \mathrm{C}$ in $10 \mathrm{~mL}$ isopropanol after the addition of two drops of saturated aqueous $\mathrm{NaHCO}_{3}$. During cooling, light-yellow crystals formed, which were separated from the gel and washed with isopropanol. This procedure was repeated until no more crystals could be separated from the gel. The product was purified by column chromatography (ethyl acetate $/ n$-pentane $1: 2 ; R_{\mathrm{f}}=0.63$ ) and dried in vacuo over $\mathrm{P}_{4} \mathrm{O}_{10}(2.07 \mathrm{mmol}, 0.50 \mathrm{~g}, 17 \%)$. ${ }^{1} \mathrm{H}$ NMR $\left(400 \mathrm{MHz}, \mathrm{CDCl}_{3}\right): \delta=7.82(\mathrm{~m}, 2 \mathrm{H}), 7.55$ $(\mathrm{m}, 1 \mathrm{H}), 7.37(\mathrm{~m}, 1 \mathrm{H}), 5.82(\mathrm{~s}, 1 \mathrm{H}), 3.10-2.84(\mathrm{~m}, 4 \mathrm{H}), 2.17-1.83(\mathrm{~m}, 2 \mathrm{H}) \mathrm{ppm} .{ }^{13} \mathrm{C} \mathrm{NMR}(100 \mathrm{MHz}$, $\left.\mathrm{CDCl}_{3}\right): \delta=133.4,130.7,129.0,124.7,46.0,32.3,25.1 \mathrm{ppm}$. Note that quaternary carbon signals were not detected. HRMS (ESI): calcd m/z for $\mathrm{C}_{10} \mathrm{H}_{11} \mathrm{NO}_{2} \mathrm{~S}_{2} \mathrm{Na}[\mathrm{M}+\mathrm{Na}]^{+} 264.0123$, found 264.0120. 


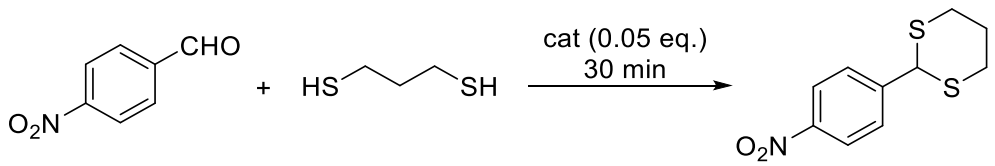

$1.81 \mathrm{~g}$ (12 mmol) 4-nitrobenzaldehyde were dissolved in $1.3 \mathrm{~mL}$ (13.2 mmol) 1,3-propane dithiol. $139.5 \mathrm{mg}$ ( $0.63 \mathrm{mmol}, 0.05$ eq.) cat were added to the yellow-green solution. After $30 \mathrm{~min}$, a yellow gel formed, which was heated to $100^{\circ} \mathrm{C}$ in $10 \mathrm{~mL}$ isopropanol after addition of two drops of saturated aqueous $\mathrm{NaHCO}_{3}$. During cooling, light-yellow crystals formed, which were separated from the gel and washed with isopropanol. This procedure was repeated until no more crystals could be separated from the gel. The product was purified by column chromatography $\left(\mathrm{DCM} ; R_{\mathrm{f}}=0.73\right)$ and dried in vacuo over $\mathrm{P}_{4} \mathrm{O}_{10}$ (3.36 mmol, $0.81 \mathrm{~g}, 28 \%) .{ }^{1} \mathrm{H}$ NMR $\left(400 \mathrm{MHz}, \mathrm{CDCl}_{3}\right): \delta=8.19(\mathrm{~m}, 2 \mathrm{H}), 7.67(\mathrm{~m}, 2 \mathrm{H}), 5.23(\mathrm{~s}, 1 \mathrm{H})$, $3.12-2.92(\mathrm{~m}, 4 \mathrm{H}), 2.25-1.91(\mathrm{~m}, 2 \mathrm{H}) \mathrm{ppm} .{ }^{13} \mathrm{C} \mathrm{NMR}\left(100 \mathrm{MHz}, \mathrm{CDCl}_{3}\right): \delta=147.8,146.1,128.9,124.0$, 50.4, 31.8, $24.8 \mathrm{ppm}$. Chemical shifts agree with a reference spectrum. ${ }^{1} \mathrm{HRMS}$ (ESI): calcd $\mathrm{m} / \mathrm{z}$ for $\mathrm{C}_{10} \mathrm{H}_{11} \mathrm{NO}_{2} \mathrm{~S}_{2} \mathrm{Na}[\mathrm{M}+\mathrm{Na}]^{+}$264.0123, found 264.0126.

\subsection{2-Nitrobenzyl- $\alpha, \alpha-d_{2}$-methanol}

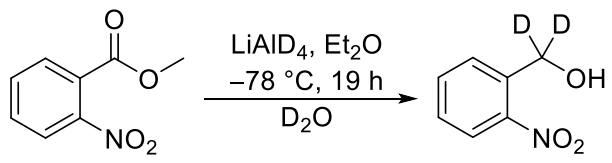

$0.30 \mathrm{~g}(7.15 \mathrm{mmol})$ lithium aluminium deuteride $\left(\mathrm{LiAlD}_{4}\right)$ were dissolved in $15 \mathrm{~mL}$ diethyl ether $\left(\mathrm{Et}_{2} \mathrm{O}\right)$ and cooled to $-78{ }^{\circ} \mathrm{C}$ with acetone $/ \mathrm{CO}_{2}$ dry ice. A mixture of $1.75 \mathrm{~mL}(12.5 \mathrm{mmol})$ methyl-2-nitrobenzoate in $5 \mathrm{~mL} \mathrm{Et}_{2} \mathrm{O}$ was added dropwise and stirred for $19 \mathrm{~h}$ while slowly reaching r. t. $\mathrm{D}_{2} \mathrm{O}$ was added until gas formation had stopped. Diluted aqueous $\mathrm{HCl}$ was added until the precipitate had dissolved. The phases were separated and the aqueous layer was extracted twice with $20 \mathrm{~mL} \mathrm{Et}_{2} \mathrm{O}$. The combined organic phases were washed with $40 \mathrm{~mL}$ brine. After evaporation of the solvent, the remaining brown solid was purified by column chromatography $\left(\mathrm{DCM} ; R_{\mathrm{f}}=0.33\right)$ and dried in vacuo over $\mathrm{P}_{4} \mathrm{O}_{10}(3.93 \mathrm{mmol}, 0.61 \mathrm{~g}$, 31\%). ${ }^{1} \mathrm{H}$ NMR $\left(400 \mathrm{MHz}, \mathrm{CDCl}_{3}\right): \delta=8.03(\mathrm{~m}, 1 \mathrm{H}), 7.66(\mathrm{~m}, 2 \mathrm{H}), 7.42(\mathrm{~m}, 1 \mathrm{H}) \mathrm{ppm} .{ }^{13} \mathrm{C}$ NMR $\left(100 \mathrm{MHz}, \mathrm{CDCl}_{3}\right): \delta=147.8,136.6,134.2,130.1,128.6,125.0 \mathrm{ppm}$. Note that the $C_{2}$ carbon signal was not detected. Chemical shifts agree with a reference spectrum. ${ }^{2}$ HRMS (ESI): calcd $\mathrm{m} / \mathrm{z}$ for $\mathrm{C}_{7} \mathrm{H}_{5} \mathrm{D}_{2} \mathrm{NO}_{3} \mathrm{Na}[\mathrm{M}+\mathrm{Na}]^{+}$178.0443, found 178.0445.

${ }^{1}$ Ong, B. S. Tetrahedron Lett. 1980, 21, 4225-4228.

${ }^{2}$ Blanc, A.; Bochet, C. G. Org. Lett. 2007, 9, 2649-2651. 


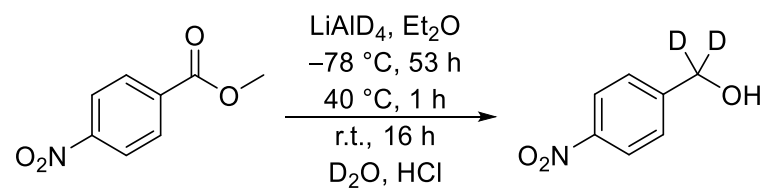

$0.30 \mathrm{~g}(7.15 \mathrm{mmol})$ lithium aluminium deuteride $\left(\mathrm{LiAlD}_{4}\right)$ were dissolved in $15 \mathrm{~mL}$ diethyl ether $\left(\mathrm{Et}_{2} \mathrm{O}\right)$ and cooled to $-78{ }^{\circ} \mathrm{C}$ with acetone $/ \mathrm{CO}_{2}$ dry ice. A mixture of $2.27 \mathrm{~g}$ (12.5 mmol) methyl-4-nitrobenzoate in $20 \mathrm{~mL} \mathrm{Et}_{2} \mathrm{O}$ was added dropwise. The mixture was stirred for $53 \mathrm{~h}$ while slowly reaching r. t., subsequently heated to $40^{\circ} \mathrm{C}$ for $1 \mathrm{~h}$, and stirred at $\mathrm{r}$. t. for $16 \mathrm{~h}$. $\mathrm{D}_{2} \mathrm{O}$ was added until gas formation had stopped. Diluted aqueous $\mathrm{HCl}$ was added until the precipitate had dissolved. The phases were separated and the aqueous layer was extracted twice with $20 \mathrm{~mL} \mathrm{Et}_{2} \mathrm{O}$. The combined organic phases were washed with $40 \mathrm{~mL}$ of brine. After evaporation of the solvent, the remaining yellow solid was purified by column chromatography (tert-butyl methyl ether; $\left.R_{\mathrm{f}}=0.28\right)$ and dried in vacuo over $\mathrm{P}_{4} \mathrm{O}_{10}(6.97 \mathrm{mmol}, 1.08 \mathrm{~g}$, $56 \%$ ). ${ }^{1} \mathrm{H}$ NMR (400 MHz, $\left.\mathrm{CDCl}_{3}\right): \delta=8.15(\mathrm{~m}, 2 \mathrm{H}), 7.48(\mathrm{~m}, 2 \mathrm{H}) \mathrm{ppm} .{ }^{13} \mathrm{C} \mathrm{NMR}\left(100 \mathrm{MHz}, \mathrm{CDCl}_{3}\right)$ : $\delta=148.0,147.4,127.1,123.8 \mathrm{ppm}$. Note that the $C_{2}$ carbon signal was not detected. Chemical shifts agree with a reference spectrum. ${ }^{3} \mathrm{HRMS}$ (ESI): calcd m/z for $\mathrm{C}_{7} \mathrm{H}_{5} \mathrm{D}_{2} \mathrm{NO}_{3} \mathrm{Na}[\mathrm{M}+\mathrm{Na}]^{+} 178.0443$, found 178.0446.

4.6. 2-Nitrobenzyl- $\alpha$ - $d$-aldehyde

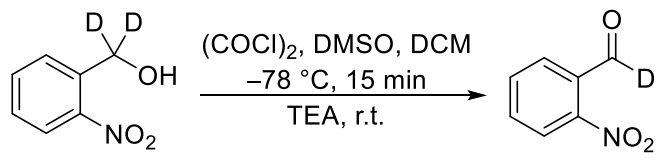

$0.5 \mathrm{~mL}(5.83 \mathrm{mmol})$ oxalyl chloride $\left[(\mathrm{COCl})_{2}\right]$ were dissolved in $10 \mathrm{~mL}$ DCM and cooled to $-78{ }^{\circ} \mathrm{C}$ with acetone $/ \mathrm{CO}_{2}$ dry ice. A mixture of ca. $0.7 \mathrm{~mL}(9.86 \mathrm{mmol})$ dimethyl sulfoxide (DMSO) in $2 \mathrm{~mL} \mathrm{DCM}$ was added. After $5 \mathrm{~min}$, a mixture of $0.6 \mathrm{~g}(3.87 \mathrm{mmol}) 2$-nitrobenzyl- $\alpha, \alpha-d_{2}$-methanol in $9 \mathrm{~mL} \mathrm{DCM}$ was added. After another $15 \mathrm{~min}, 3 \mathrm{~mL}(21.64 \mathrm{mmol})$ triethyl amine (TEA) were added to the brown solution. After warming the reaction mixture to r. t., $15 \mathrm{~mL}$ water were added. The phases were separated and the aqueous phase was extracted with $15 \mathrm{~mL}$ DCM. The combined organic phases were subsequently washed with $45 \mathrm{~mL}$ brine, $15 \mathrm{~mL}$ diluted $\mathrm{H}_{2} \mathrm{SO}_{4}, 15 \mathrm{~mL}$ water, and $15 \mathrm{~mL}$ saturated aqueous $\mathrm{NaHCO}_{3}$. After evaporation of the solvent, the remaining brown-yellow solid was purified by column chromatography (ethyl acetate; $\left.R_{\mathrm{f}}=0.75\right)$ and dried in vacuo over $\mathrm{P}_{4} \mathrm{O}_{10}(3.03 \mathrm{mmol}, 0.46 \mathrm{~g}, 78 \%) .{ }^{1} \mathrm{H}$ NMR $(400$ $\left.\mathrm{MHz}_{,} \mathrm{CDCl}_{3}\right): \delta=8.05(\mathrm{~m}, 1 \mathrm{H}), 7.89(\mathrm{~m}, 1 \mathrm{H}), 7.72(\mathrm{~m}, 2 \mathrm{H}) \mathrm{ppm} .{ }^{13} \mathrm{C} \mathrm{NMR}\left(100 \mathrm{MHz}, \mathrm{CDCl}_{3}\right): \delta=134.1$, $133.7,129.7,124.5 \mathrm{ppm}$. Note that quaternary and $C O$ carbon signals were not detected. HRMS (ESI): calcd $\mathrm{m} / \mathrm{z}$ for $\mathrm{C}_{8} \mathrm{H}_{8} \mathrm{DNO}_{4} \mathrm{Na}[\mathrm{M}+\mathrm{MeOH}+\mathrm{Na}]^{+} 207.0486$, found 207.0490.

${ }^{3}$ Price, K. E.; Broadwater, S. J.; Walker, B. J.; McQuade, D. T. J. Org. Chem. 2005, 70, 3980-3987. 


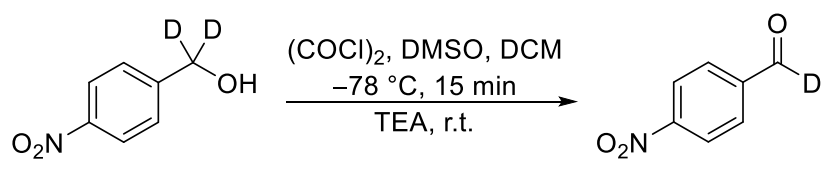

$0.6 \mathrm{~mL}(7.00 \mathrm{mmol})$ oxalyl chloride $\left[(\mathrm{COCl})_{2}\right]$ were dissolved in $15 \mathrm{~mL} \mathrm{DCM}$ and cooled to $-78^{\circ} \mathrm{C}$ with acetone $/ \mathrm{CO}_{2}$ dry ice. A mixture of $1 \mathrm{~mL}(14.1 \mathrm{mmol})$ dimethyl sulfoxide (DMSO) in $3 \mathrm{~mL}$ DCM was added. After $5 \mathrm{~min}$, a mixture of $1.0 \mathrm{~g}(6.45 \mathrm{mmol})$ 4-nitrobenzyl- $\alpha, \alpha$ - $d_{2}$-methanol in $15 \mathrm{~mL}$ DCM was added. After another $15 \mathrm{~min}, 4.5 \mathrm{~mL}(32.51 \mathrm{mmol})$ triethyl amine (TEA) were added to the yellow solution. After warming the reaction mixture to r. t., $30 \mathrm{~mL}$ water were added. The phases were separated and the aqueous phase was extracted with $30 \mathrm{~mL}$ DCM. The combined organic phases were subsequently washed with $70 \mathrm{~mL}$ brine, $30 \mathrm{~mL}$ diluted $\mathrm{H}_{2} \mathrm{SO}_{4}, 30 \mathrm{~mL}$ water, and $30 \mathrm{~mL}$ saturated aqueous $\mathrm{NaHCO}_{3}$. After evaporation of the solvent, the remaining yellow solid was purified by column chromatography (ethyl acetate; $\left.R_{\mathrm{f}}=0.78\right)$ and dried in vacuo over $\mathrm{P}_{4} \mathrm{O}_{10}(5.06 \mathrm{mmol}, 0.77 \mathrm{~g}, 78 \%)$. ${ }^{1} \mathrm{H} \mathrm{NMR}(400 \mathrm{MHz}$, $\left.\mathrm{CDCl}_{3}\right): \delta=8.32(\mathrm{~m}, 2 \mathrm{H}), 8.03(\mathrm{~m}, 2 \mathrm{H}) \mathrm{ppm} .{ }^{13} \mathrm{C} \mathrm{NMR}\left(100 \mathrm{MHz}, \mathrm{CDCl}_{3}\right): \delta=130.5,124.3$ ppm. Note that quaternary and $\mathrm{CO}$ carbon signals were not detected. HRMS (ESI): calcd $\mathrm{m} / \mathrm{z}$ for $\mathrm{C}_{8} \mathrm{H}_{8} \mathrm{DNO}_{4} \mathrm{Na}_{[\mathrm{M}}$ $+\mathrm{MeOH}+\mathrm{Na}]^{+}$207.0486, found 207.0490.

4.8. 2-Deutero-2-(2-nitrophenyl)-1,3-dithiane

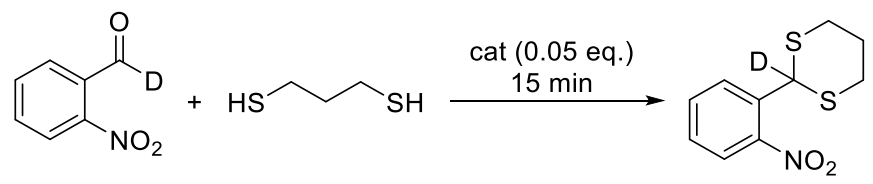

$0.21 \mathrm{~g}$ (1.38 mmol) 2-nitrobenzyl- $\alpha$ - $d$-aldehyde were dissolved in $0.15 \mathrm{~mL}$ (1.49 mmol) 1,3-propane dithiol. $16.5 \mathrm{mg}(0.07 \mathrm{mmol}, 0.05$ eq. $)$ cat were added to the brown solution. After $15 \mathrm{~min}$, a brown gel formed, which was heated to $100{ }^{\circ} \mathrm{C}$ in ca. $3 \mathrm{~mL}$ isopropanol after addition of two drops of saturated aqueous $\mathrm{NaHCO}_{3}$. During cooling, light-yellow crystals formed, which were separated from the gel and washed with isopropanol. This procedure was repeated until no more crystals could be separated from the gel. The product was purified by column chromatography (ethyl acetate $/ n$-pentane $1: 2 ; R_{\mathrm{f}}=0.60$ ) and dried in vacuo over $\mathrm{P}_{4} \mathrm{O}_{10}(0.62 \mathrm{mmol}, 0.15 \mathrm{~g}, 20 \%) .{ }^{1} \mathrm{H}$ NMR $\left(400 \mathrm{MHz}, \mathrm{CDCl}_{3}\right): \delta=7.88(\mathrm{~m}, 2 \mathrm{H}), 7.62$ $(\mathrm{m}, 1 \mathrm{H}), 7.44(\mathrm{~m}, 1 \mathrm{H}), 3.17-2.90(\mathrm{~m}, 4 \mathrm{H}), 2.23-1.94(\mathrm{~m}, 2 \mathrm{H}) \mathrm{ppm} .{ }^{13} \mathrm{C} \mathrm{NMR}\left(100 \mathrm{MHz}, \mathrm{CDCl}_{3}\right): \delta=$ $133.5,130.8,129.1,124.8,32.3,25.0 \mathrm{ppm}$. Note that quaternary and $C D$ carbon signals were not detected. HRMS (ESI): calcd m/z for $\mathrm{C}_{10} \mathrm{H}_{10} \mathrm{DNO}_{2} \mathrm{~S}_{2} \mathrm{Na}[\mathrm{M}+\mathrm{Na}]^{+} 265.0186$, found 265.0183. 


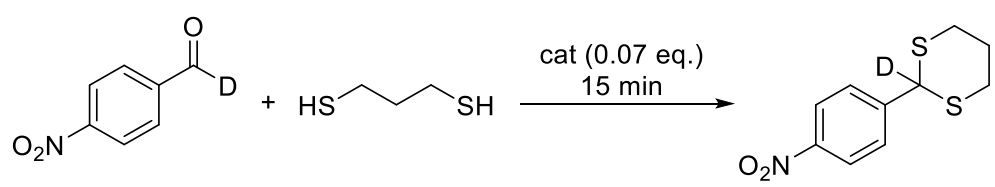

$0.20 \mathrm{~g}$ (1.31 mmol) 4-nitrobenzyl- $\alpha$ - $d$-aldehyde were dissolved in $0.15 \mathrm{~mL}$ (1.49 mmol) 1,3-propane dithiol. $19.0 \mathrm{mg}(0.09 \mathrm{mmol}, 0.07$ eq. $)$ cat were added to the brown solution. After $15 \mathrm{~min}$, a yellow gel formed, which was heated to $100{ }^{\circ} \mathrm{C}$ in ca. $3 \mathrm{~mL}$ isopropanol after addition of two drops of saturated aqueous $\mathrm{NaHCO}_{3}$. During cooling, light-yellow crystals formed, which were separated from the gel and washed with isopropanol. This procedure was repeated until no more crystals could be separated from the gel. The product was purified by column chromatography (ethyl acetate $n$-pentane $1: 2 ; R_{\mathrm{f}}=0.49$ ) and the crystals were dried in vacuo over $\mathrm{P}_{4} \mathrm{O}_{10}(1.53 \mathrm{mmol}, 0.37 \mathrm{~g}, 31 \%) .{ }^{1} \mathrm{H}$ NMR $\left(400 \mathrm{MHz}, \mathrm{CDCl}_{3}\right): \delta=8.19$ $(\mathrm{m}, 2 \mathrm{H}), 7.66(\mathrm{~m}, 2 \mathrm{H}), 3.12-2.92(\mathrm{~m}, 4 \mathrm{H}), 2.25-1.92(\mathrm{~m}, 2 \mathrm{H}) \mathrm{ppm} .{ }^{13} \mathrm{C} \mathrm{NMR}\left(100 \mathrm{MHz}, \mathrm{CDCl}_{3}\right)$ : $\delta=147.8,146.1,128.9,124.1,31.7,24.8 \mathrm{ppm}$. Note that the $C D$ carbon signal was not detected. HRMS (ESI): calcd m/z for $\mathrm{C}_{10} \mathrm{H}_{10} \mathrm{DNO}_{2} \mathrm{~S}_{2} \mathrm{Na}[\mathrm{M}+\mathrm{Na}]^{+}$265.0186, found 265.0184.

4.10. 4-Nitrobenzaldehyde dimethyl bisthioacetal

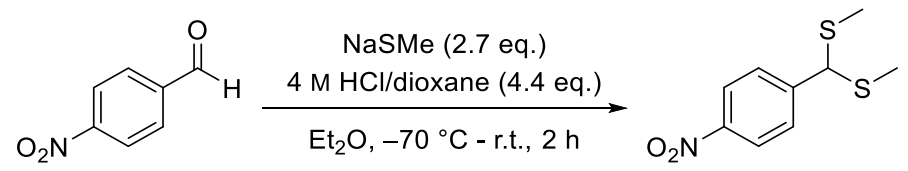

Sodium methanethiolate $(372.0 \mathrm{mg}, 5.3 \mathrm{mmol})$ was suspended in $\mathrm{Et}_{2} \mathrm{O}(5 \mathrm{~mL})$ and cooled to $-70{ }^{\circ} \mathrm{C}$. $\mathrm{HCl}$ in dioxane $(4 \mathrm{M}, 2.2 \mathrm{~mL}, 8.8 \mathrm{mmol})$ was added dropwise with stirring followed by a solution of $p$-nitrobenzaldehyde $(302.0 \mathrm{mg}, 2.0 \mathrm{mmol})$ in $\mathrm{Et}_{2} \mathrm{O}(15 \mathrm{~mL})$. The reaction mixture was allowed to slowly warm to r. t. and stirred for further $2 \mathrm{~h}$. Saturated aqueous $\mathrm{NaHCO}_{3}(10 \mathrm{~mL})$ was then added and the reaction mixture was diluted with $\mathrm{Et}_{2} \mathrm{O}$. The phases were separated and the organic layer was washed with saturated aqueous $\mathrm{NaHCO}_{3}(15 \mathrm{~mL})$ and brine $(15 \mathrm{~mL})$. Drying over $\mathrm{Na}_{2} \mathrm{SO}_{4}$, removal of the solvent under reduced pressure, and column chromatography (hexane/EtOAc 2:1; $R_{\mathrm{f}}=0.59$ ) afforded $74.0 \mathrm{mg}$ $(0.32 \mathrm{mmol}, 16 \%)$ of the desired product as a yellow solid. ${ }^{1} \mathrm{H}$ NMR $\left(400 \mathrm{MHz}, \mathrm{CDCl}_{3}\right): \delta=8.20(\mathrm{~m}$, 2H), $7.59(\mathrm{~m}, 2 \mathrm{H}), 4.83(\mathrm{~s}, 1 \mathrm{H}), 2.12(\mathrm{~s}, 6 \mathrm{H}) \mathrm{ppm} .{ }^{13} \mathrm{C}$ NMR $\left(100 \mathrm{MHz}, \mathrm{CDCl}_{3}\right): \delta=147.5,147.3,128.7$, 124.0, 55.8, $15.0 \mathrm{ppm}$. HRMS (ESI): calcd m/z for $\mathrm{C}_{9} \mathrm{H}_{11} \mathrm{NO}_{2} \mathrm{~S}_{2} \mathrm{Na}[\mathrm{M}+\mathrm{Na}]^{+} 252.0123$, found 252.0125. 